Portland State University

PDXScholar

Dissertations and Theses

Dissertations and Theses

Summer 8-8-2019

\title{
Engaging in a Rural Deaf Community of Practice
}

Kara Gournaris

Portland State University

Follow this and additional works at: https://pdxscholar.library.pdx.edu/open_access_etds

Part of the Curriculum and Instruction Commons, First and Second Language Acquisition Commons, and the Higher Education Commons

Let us know how access to this document benefits you.

\section{Recommended Citation}

Gournaris, Kara, "Engaging in a Rural Deaf Community of Practice" (2019). Dissertations and Theses. Paper 5114.

https://doi.org/10.15760/etd.6993

This Dissertation is brought to you for free and open access. It has been accepted for inclusion in Dissertations and Theses by an authorized administrator of PDXScholar. Please contact us if we can make this document more accessible: pdxscholar@pdx.edu. 
Engaging in a Rural Deaf Community of Practice

by

Kara Gournaris

A dissertation submitted in partial fulfillment of the requirements for the degree of

\author{
Doctor of Education \\ in \\ Educational Leadership: Curriculum and Instruction
}

\begin{abstract}
Dissertation Committee:
Micki M. Caskey, Chair

Anita Bright

Dot McElhone

Amy Donaldson
\end{abstract}

Portland State University

2019 
(C) 2019 Kara Gournaris 


\begin{abstract}
The number of students taking American Sign Language (ASL) at the postsecondary level continues to increase as more Deaf-related graduate programs and employment settings require fluent ASL skills. Western Oregon University (WOU) is one of the few existing programs in the United States that offers four years of ASL instruction; however, as a rural university it has limited access to a Deaf community. The problem of practice is that students often have little exposure to rich language models who are fluent in ASL, which impacts their legitimate peripheral participation (LPP) in the local Deaf communities of practice (CoPs) and reduces apprenticeship opportunities that might be beneficial for their language acquisition experiences. While research suggests that direct engagement with language models has a significant impact on language acquisition and fluency in general, there is scant literature in the field of ASL that addresses this connection. My dissertation research addressed this gap in the literature. To address my problem of practice, I used a qualitative research design with both descriptive and comparative research questions to complete three case studies. Using semi-structured interviews, this study explored participants' attitudes and beliefs about their experience in local Deaf CoPs. The three participants in my study were Western Oregon University students enrolled in ASL 8 course. Data analyses included inductive analysis of qualitative data, a within case analysis, and a cross-case analysis. Findings showed that participants were interested in moving past LPP in local Deaf CoPs, and were interested in identifying apprenticeship opportunities because they realized the importance of engaging with native language models.
\end{abstract}




\section{Dedication}

To my love, Heather Jane. You have always believed in me and told me I could do anything. Over the past three years, when everything in our world changed because I went back to school, you stayed by my side. You sat right next to me every night while I completed my assignments and stayed up with me until the wee hours of the morning as I worked on my dissertation. You knew your presence next to me made a difference. No matter how busy things got, you always made sure we kept our Saturday nights free for date night!

Throughout this entire journey, never once did you stop believing in me. When I became overwhelmed at the mountains ahead of me, you reminded me to take one step at a time. You have always been my rock. Thank you for your continued love and support. You will always be the love of my life. I love you! 


\section{Acknowledgements}

My journey through this doctoral program would not have been as transformative and life changing without the amazing people who have shared these moments with me. First, I would like to express my most sincere appreciation to the participants of my study. Your willingness to share your experiences with me, and your trust that I would take your stories to heart, means more to me than I can express. I will never forget your bravery, and your conscious choice to engage with me in these conversations. You will always have a place in our Deaf community.

Thank you to my dissertation committee members, Micki, Anita, Dot, and Amy for your open minds, loving hearts, and your contagious curiosity. Your questions inspired me to dig deeper and really focus on the story I wanted to tell. I am overcome with gratitude for the people at PSU who have become like family to me. Micki and Anita, you challenged my old frames and gave me the tools I needed to find my voice. You gave me the gift of research, and I promise to use that gift in service of my community. I am a better educator and person because of you both.

To the members of my cohort, thank you for the countless hours of deep connection and conversation, lots of laughter, tears, and love. I know you will all go on to change your corners of the world. I am so proud to know each of you and will always be in your corner.

This experience would not have been possible without the amazing team of interpreters who allowed me to bridge the hearing and Deaf worlds. Hal, Jake, and Jenna, 
I am grateful for your commitment for the past three years. You brought communication to life.

None of this work would have been possible, or meaningful, without my colleagues at Western Oregon University. To the ASL Studies team: Katie, Lyra, Tie, Brent, and Jolene, I could not have done this work without you. Your passion, persistence, and presence makes all the difference in the lives of the students we teach. Thank you for being the best colleagues I could ever ask for. To Dean Mark Girod, I am grateful that Hank Bersani led me to you. Your belief that I could complete a doctoral program led me to believe in myself. Dr. Denise Thew Hackett, thank you for always checking in and for the reminder to keep my eye on the prize.

A heart full of gratitude to my parents and brothers for being my first example of the power of the Deaf community. Thank you for the gift of our language, culture, and instilling in me the belief that I can do anything. Being part of our family has taught me the value of hard work, the importance of commitment, and helped shape me into the woman I am today. Thank you for the rest of my family and friends who never doubted me, and whose faith in me made all the difference.

To Julia Smith, you were my inspiration to start this journey, and every day I am grateful for the gift of your presence in my life. You are my mentor, my confidant, and my treasured friend. Thank you from the bottom of my heart.

To my kids, Sha and Trayshun, thank you for believing that what I do makes a difference. I know it was hard for me to be away and always busy, but you never complained because you knew this journey was important to me. 
All my love and gratitude to my fur babies who made sure I had plenty of love even on my most stressful days. 
Table of Contents

Abstract

Chapter 1: Introduction ............................................................................

Background of the Problem of Practice ............................................................ 7

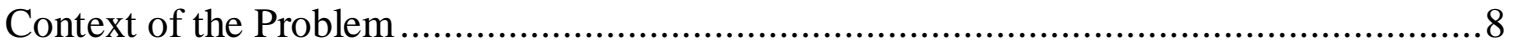

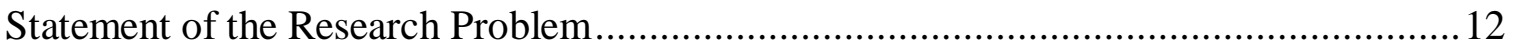

Significance of the Research Problem ............................................................. 15

Presentation of Methods and Research Question .............................................. 16

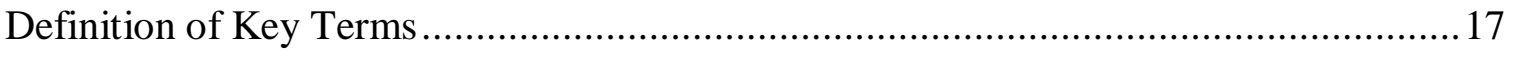

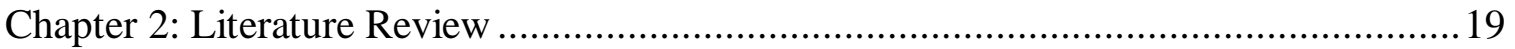

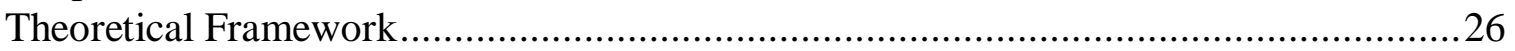

Review of the Research Literature ...................................................................... 45

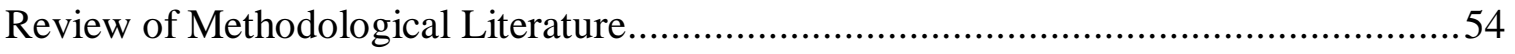

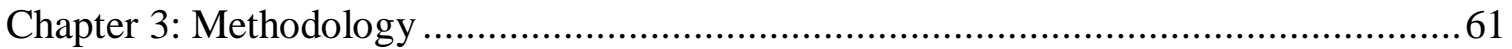

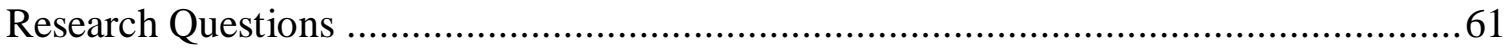

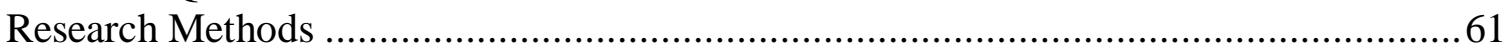

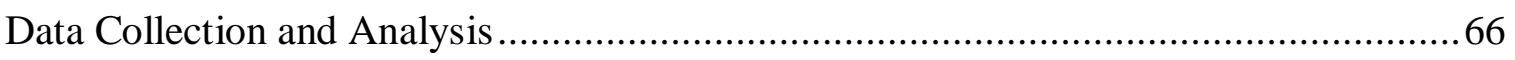

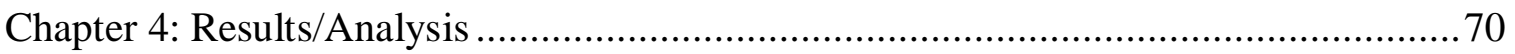

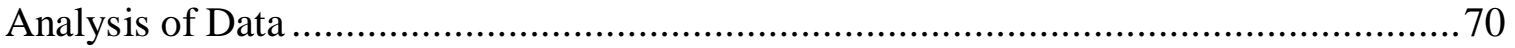

Findings Related to Research Questions ................................................................93

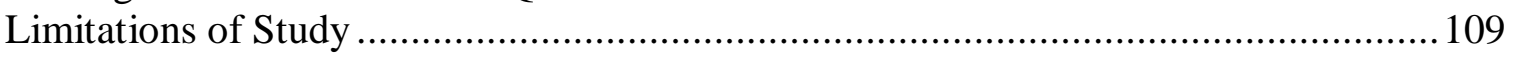

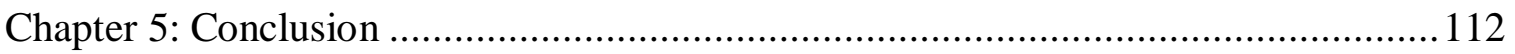

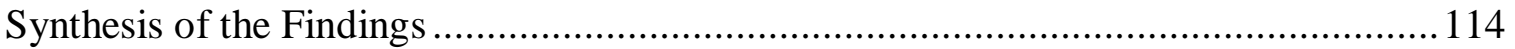

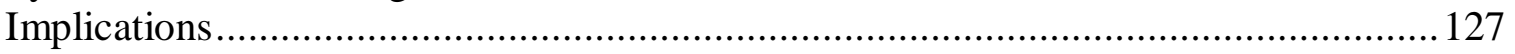

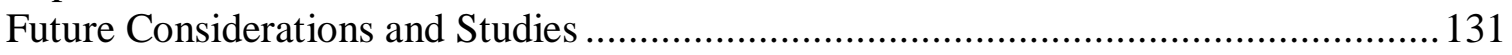

Research in Other Rural Communities .............................................................. 132

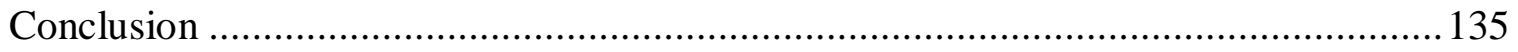

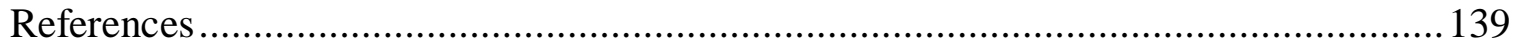




\section{Appendices}

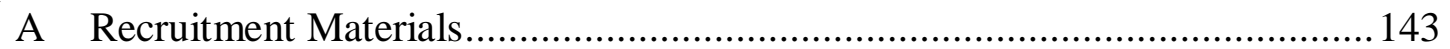

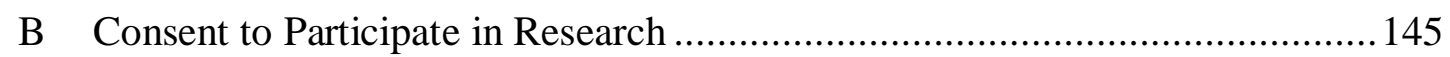

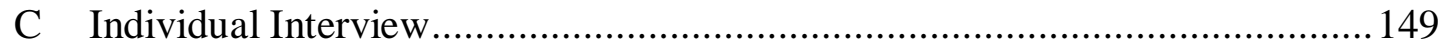


List of Figures

Figure $1 \quad$ Deaf CoP Model...................................................................... 14 


\section{Chapter 1: Introduction}

For centuries, Deaf people fought for the right to communicate in a language that was accessible, and to be part of a community that shared similar cultural norms and values. Due to a longstanding history of oppression from hearing people who held both power and privilege over the Deaf community, American Sign Language (ASL) was not recognized as an official language until 1960 (Stokoe, 2005), even though sign language has been used in some form in North America since at least the 1500s. William Stokoe, a researcher and linguist, demonstrated ASL was a complex language complete with grammar, syntax, semantics, and other linguistic properties (Armstrong, 2000). Prior to his work in the 1950s, the general population of hearing people believed ASL was nothing more than an English-based system of hand movements. Stokoe developed a transcription system to document that ASL was a complete language, and in 1960, his ideas appeared in a published monograph and linguists finally accepted his system. Stokoe's work had far-reaching effects in the field of Deaf Education and later in the instruction of ASL as a foreign language (Armstrong, 2000).

To put the advent of ASL into perspective, educators accepted ASL as a language 17 years before I was born. My parents, who are both Deaf, grew up using a language that others did not consider a real language until they were in their 20s. While ASL is common in schools and universities across the country now, this was not the case 50-60 years ago. Strong opposition to the use of ASL was rampant and generations of Deaf people are still feeling the impact in 2018. Hearing educators, physicians, and other 
professionals believed using ASL would prevent a Deaf person from learning to read, write, and speak English, so they forbid the use of ASL for many years. Not until Deaf people came into their own power, and fought for the right to use ASL, did this paradigm begin to shift, but there are still pockets of educators and physicians in 2018 who believe that ASL is inferior to English and that Deaf people need remediation. To illustrate this point further, we need only examine the Deaf faculty in the ASL Studies program at Western Oregon University (WOU). Of nine Deaf faculty at WOU, I am the only one who has an extended Deaf family (e.g., parents, aunts, and siblings) and who grew up with ASL as my native language. I attended a residential school for the Deaf and graduated from Gallaudet University; both settings provided a critical mass of educated Deaf peers and role models from whom I could learn.

While I had exposure to English growing up, my primary language of instruction and conversation was ASL. My colleagues grew up varying degrees of access to ASL because they grew up with hearing families and in mainstream school settings where their language models were most commonly hearing interpreters who learned ASL as a second language. Some of their families learned to communicate using ASL, but most never became fluent and therefore my peers seldom had access to fluent ASL language models. While all nine of us faculty members in in the ASL Studies program at WOU now identify as Deaf, our experiences and exposure to ASL and the Deaf community could not be more different. It is important to understand this nuance because personal experience and language fluency influences our practice as we teach ASL as a modern language to students at WOU. 
To live in a time where professionals accept ASL as a language, and where hearing people are eager to learn it, is a surreal experience for me. This was not my experience growing up from 1980-2000. During that time my family was often made fun of for being Deaf and using ASL, and we were often denied our rights to an interpreter or any other accommodation, and until the 1990s it was legally permissible for people to openly discriminate against Deaf people in employment, housing, educational settings, and business. While there has been so much improvement, there are still communities where Deaf people are denied the use of ASL. For example, oral school programs may not allow the use of ASL. This makes me wonder why hearing students' decision to learn ASL is acceptable and often commended, while many hearing educators and professional often deny Deaf people the right to an accessible language because they contend that the use of ASL will impede their learning. The exploration of first language acquisition is beyond the scope of this dissertation, but it is important to understand that hearing students have more opportunities to learn ASL than their Deaf counterparts in many instances do. While some high schools offer ASL classes for hearing students, many residential schools for the Deaf do not offer ASL classes. So, while Deaf students receive instruction in sign language, they never take courses to learn more about their own language, grammar, syntax, semantics, poetry, storytelling techniques, or other important features that make ASL different than English. This would be similar to hearing students growing up in the United States and never taking English classes. This affects the Deaf community and perpetuates the inequality that already exists. This limited level of access 
to their own language further perpetuates a system where hearing people have more opportunities than Deaf people do, even when it comes to learning and teaching ASL.

WOU is located in the small, unassuming town of Monmouth, Oregon. This rural university is home to approximately 6,000 students, with an average of 370 students enrolled in ASL courses each year. The ASL Studies faculty at WOU consists of five full-time and five part-time instructors, nine of whom are Deaf and use ASL as their primary means of communication. When visiting WOU's campus, you may see students and instructors using ASL to communicate. ASL is the largest modern language on campus, and classes taught in this program include ASL 1-9 courses: ASL Linguistics, Mental Health in the Deaf Community, Sociolinguistics of Deaf Communities, American Deaf Culture, and 14 other courses related to ASL and/or the Deaf community.

While most community colleges, colleges, and university programs offer two years of ASL instruction focused on the acquisition of ASL, WOU's program offers four years of coursework that encompasses more than only learning about the language. Due to the limited number of ASL Studies programs in North America that offer four years of ASL coursework, a large number of students start their education at other institutions and transfer to WOU to complete their ASL studies courses before enrolling in a graduate program or finding employment in a Deaf-related field.

WOU has a large population of students who study sign language due to the popularity of the ASL Studies, Deaf Education, Interpreting Studies Program, and the Mental Health and Rehabilitation Counseling programs. WOU is one of the few institutions across the country that offers a bachelor's degree in ASL Studies. The ASL 
Studies program adheres to an immersion-based philosophical approach, so instructors teach all courses in ASL. For students who enroll in ASL related courses (e.g., Deaf History, Mental Health in the Deaf Community) but who do not know ASL, interpreters are available to provide sign-to-voice interpretation. Otherwise, students are encouraged to refrain from communicating with one another in spoken English directly before, during, or directly after their ASL course. This is to encourage students to practice their conversational ASL skills in addition to their academic ASL skills. According to Vygotsky's (1978) socio-cultural theory, social interactionist theory examines how students acquire language. Drawing from socio-cultural theory, Lave and Wenger (1991) developed situated learning theory, which suggests that learning happens incidentally by interaction with others in natural and authentic settings and contexts called communities of practice (CoPs). Brown, Collins, and Duguid (1989) argued that incidental learning needs to be paired with classroom instruction in what they term "cognitive apprenticeship" (p. 32) and that students should have access to both informal and classroom-based instruction for optimal learning.

In efforts to achieve this cognitive apprenticeship and to simulate an immersion environment where students, faculty, and community members meet to practice ASL and develop ongoing relationships, WOU established a campus-sponsored ASL Club. The main goal of ASL Club is to introduce new language learners to native language users (Deaf and Hard of Hearing people) so students have an opportunity to use their new language skills and learn more about the Deaf community through interaction. This is a 
CoP model, where students interact and develop relationships with Deaf community members.

Since about 2016, the ASL program at WOU has seen a decrease in the number of students who voluntarily attend ASL Club regularly, because most students have other commitments that prevent them from spending extra time on campus each week. The students who regularly attend ASL Club tend to have fewer obligations and responsibilities than those who do not attend. The social opportunities to practice the language and interact with Deaf faculty, students, and peers, are essential for natural language acquisition, but many students are unable to attend. Faculty designed all of the ASL courses with the premise that students learn new language skills (knowledge and comprehension) during our face-to-face class time, and then practice applying those skills in CoPs-based settings outside of the classroom. Based on decreased participation over the past several years in these CoP-based settings, the ASL Studies department anticipates that this trend will continue and that our department will need to find alternative ways to provide language-rich interactions for students, because many graduate programs require students to have a minimum level of ASL fluency for admittance.

At WOU, three graduate programs currently require an advanced level of ASL proficiency: Deaf Education, the Rehabilitation Counseling and Mental Health Program, and the Interpreter Education Program. If students graduating from our undergraduate ASL program are not leaving with the fluency necessary for admission to graduate level programs, the number of WOU students entering these specific graduate level programs 
might decrease. As the majority of the faculty in the ASL Studies program is Deaf, another important consideration is that many of the students we teach continue on to become interpreters, counselors, teachers, and other professionals we must work with in our daily lives after they complete their respective programs. If we do not provide quality instruction to students, and opportunities for them to engage in a CoP within the Deaf community, we might see a decrease in the number of service providers in the field, or decreased skill level of those providers. Both of these situations will affect our faculty and the Deaf community, personally and professionally.

\section{Background of the Problem of Practice}

Students learn ASL during their ASL-specific coursework, but also need to complete a certain amount of contact hours (i.e., language use) outside of the classroom per week and attend several Deaf events each term for credit. While some areas of the United States have larger populations of Deaf people, other areas have smaller communities. Oregon does not have a large Deaf community, so the options for social events for students are far fewer than in bigger communities. For a student who is on a tight schedule, this participation with fluent users of ASL outside of class meeting times could mean the difference between getting full credit in the course and losing points for this required activity. For students attending programs in rural settings, such as WOU, this might mean students have to drive 60 miles or more to find a Deaf event with a critical mass of Deaf people. The problem of practice is that students often have little exposure to rich language models who are fluent in ASL, which impacts their LPP in the 
local Deaf CoPs, and reduces apprenticeship opportunities that might be beneficial for their language acquisition experiences.

This problem is not unique to WOU. Anecdotally, many ASL instructors from across the United States who work at various universities and colleges use social media and list serves to note the issue of limited language models and the need for greater ASL fluency. However, because most programs only offer two years of ASL coursework, a critical mass of people have not devoted time and energy to work on this problem. The problem of practice is that students often have little exposure to rich language models who are fluent in ASL, which impacts their LPP in the local Deaf CoPs and reduces apprenticeship opportunities that might be beneficial for their language acquisition experiences. While research suggests that direct engagement with language models has a significant impact on language acquisition and fluency in general, there is very little literature in the field of ASL that addresses this connection. My dissertation research addresses this gap in the literature.

\section{Context of the Problem}

According to the Modern Language Association's 2013 report, ASL is the fourth most studied language in the United States with nearly 110,000 undergraduate and graduate students enrolled in postsecondary education level ASL courses (Goldberg, Looney, \& Lusin, 2015). The number of students taking ASL as a modern language has increased significantly at WOU in recent years. Since 2010, the ASL Studies program grew from 173 students to approximately 370 students, for an increase of $113 \%$. While WOU's administration and ASL Studies department are excited to have a growing 
program, this growth puts pressure on the faculty to meet the needs of a large number of students who come from many different backgrounds in terms of their exposure to ASL and the Deaf community.

Curriculum for teaching ASL at the post-secondary level is limited. Most programs across the United States use one of two curriculums: ASL Signing Naturally (Smith, Lentz, \& Mikos, 2008) or The Green Book (Cokley \& Baker-Shenk, 1991). These authors designed both of these curricula for two-year programs because most students take ASL as a foreign language, which only requires two years of course work. To date, there is no compiled and published curriculum for the third and fourth year of ASL instruction, so most programs are on their own when selecting materials for third and fourth year students. For the majority of students take ASL to fulfill a language requirement, two years is sufficient. As the fields related to Deafness (Deaf Education, Deaf Studies, ASL Linguistics, Interpreting, Vocational Rehabilitation) continue to expand, more course work is needed to prepare students for graduate studies and work in Deaf-related fields.

From 2012-2018, the ASL Studies department at WOU has changed the materials used to teach the third and fourth year of ASL several times, even though the goals and objectives of the program remain the same. Due to the limited number of instructional materials for higher-level ASL courses, instructors at WOU have had to piece together materials to use each term. To this end, WOU faculty developed some materials and modified other existing materials to fit higher-level courses. Changing materials each term has some disadvantages in terms of consistency of the content and skills taught to 
students each year. This inconsistency can affect students' ASL fluency, and could lead to fewer opportunities to gain access into graduate programs or find employment in Deafrelated fields.

Unfortunately, there is no nationally recognized standard assessment for ASL proficiency across all fields. Some institutions, programs, and employers use the Sign Language Proficiency Interview (SLPI) administered by National Technical Institute for the Deaf, and others use the American Sign Language Proficiency Interview (ASLPI) administered by Gallaudet University. The goal of both interview assessments is to measure global ASL skills, but neither measure language specific to a field of study. ASLPI assigns interviewees a level of competency and does not provide any feedback to the participant or instructor on why the student received the assigned score. The SLPI assigns a score and gives detailed analysis on why the student received that score and instructors and/or students can use that feedback to focus on specific areas of improvement. Some states have their own independent ASL proficiency exams for high school teachers, but the majority of states do not. With different systems and measurements in place, it is difficult for institutions, programs, and employers to know which is the most reliable and accurate. If one nationally recognized assessment existed across all fields, it would help improve standardization across ASL programs, and could lead to easier transitions for students and instructors. For example, if a student was interested in attending a graduate program at WOU and that program had a minimum competency for admittance, the student could take the proficiency interview and know if his language skills were proficient enough to apply to the graduate program. If that same 
student wanted to apply to three different programs across the United States, all in the same field, all three programs might require a different assessment or score, and this can be difficult for students to try to navigate. These types of frustrations can lead students to feel overwhelmed and deter them from continuing their education.

Language proficiency is not the only barrier students' face when applying for continuing education programs at WOU. In addition to proficiency, many students at WOU have lamented that they have little real-life experience or hands-on application in the field they want to study. As many programs require students to have experience, students often struggle with meeting this expectation.

Historically, many of the students in the ASL Studies program at WOU took ASL as a modern language. These students were typically undergraduates who only took ASL 1-6 (first and second year ASL courses) to satisfy WOU's modern language requirement. As more jobs in the field of Deafness started requiring advanced degrees, students realized they needed additional coursework to meet the increased language requirements and proficiencies. In 2012, the third and fourth year ASL courses were small: three sections of 18 students each year. As of 2017, the ASL Studies Department offers four sections of 22 students (maximum) each year and often has a waiting list for these courses. WOU is one of the few institutions on the west coast that offers advanced ASL coursework, and we see an increase in the number of students who transfer to WOU for these courses. These transfer students make up half of the students enrolled in the ASL Studies program at WOU, and their unique needs are different from those of students who started their postsecondary education journey at WOU. 
Even though our ASL faculty members recognize that a large percentage of our students are transferring in from other institutions, we have not changed our instruction/pedagogy to meet their unique needs

\section{Statement of the Research Problem}

The purpose of this research was to explore attitudinal and situational barriers that make it difficult for students to engage actively in a CoP within the Deaf community. According to Cornell-Swanson (2001), building relationships and getting involved in activities/events are key components of learning ASL. For purposes of my research, interaction with fluent language models is called contact hours. If students do not have the opportunity to interact with language models during contact hours, this might have an impact on their fluency and proficiency. I explored this problem from two different lenses: learning (academic) and acquisition (incidental learning in a CoP) of ASL.

If a student wants to continue on to a graduate level program that has a specific level of ASL fluency as a prerequisite, admission might not be granted to a student who is not proficient in ASL. At WOU, three graduate programs currently require an advanced level of ASL proficiency: Deaf and Hard of Hearing Educator program, the Rehabilitation Counseling and Mental Health program, and the Masters in Interpreting Studies program. The Deaf and Hard of Hearing Educator program requires a minimum of ASLPI score of 3 or an SLPI score of Advanced. Both the Rehabilitation Counseling and Mental Health and Masters in Interpreting Studies programs require ASL proficiency, but they do not require students to take a specific assessment. Instead of using a standardized measure, faculty determine if students' language skills are sufficient 
during the interview process. Additionally, they consider students' grades and overall grade point average.

Historically, approximately half of the admitted students to these three graduate programs have completed their undergraduate degrees at WOU, so this approach to admitting students based on grade rather than a standardized assessment has not proven to be overly problematic because program coordinators know the level of fluency students typically have when completing ASL coursework at WOU. The other half of students who apply to these programs come from a variety of backgrounds, so faculty cannot assume their language proficiency and fluency. Without a standardized approach to measuring language proficiency, it can be difficult for students to know if their language skills are sufficient for admission to their program of choice.

Access to quality ASL instruction is not the only factor that influences students' language acquisition and fluency. Exposure to, and interaction with, a native Deaf CoP also plays an important role in language development. Lave and Wenger (1991) attest that students experience LPP as they begin to interact with a new CoP. When a student first learns ASL, they are encouraged to attend Deaf events that are open to the public and that allow students access only to what happens at the periphery (outermost circle) of the community. The more interaction the student has, the more integrated they become in the CoP. Students who want to interact with the Deaf community must be willing to work on their ASL skills in order to communicate with Deaf people effectively. If a student relies only on the information learned in ASL courses, they will miss opportunities for meaningful connection in this new CoP. The more involved a student (newcomer) 
becomes in the community, and the more connected the student becomes with a Deaf community member (old timer), the more access that student has to what happens within the community that is beyond the periphery. The old timer invites the newcomer into more intimate circles of the community.

Unlike taking ASL courses for credit, where the motivation to improve skill level may be related to receiving a good grade, involvement in a native Deaf CoP may depend more on intrinsic motivation, because this participation requires students to make time to engage with native language users. While ASL instructors do require students to complete a certain number of hours engaging in Deaf events each term, simply showing up to an event does not necessarily mean students' language skills will improve. Students must have an internal desire to connect with others in the Deaf community in order to progress through the stages (see Figure 1) of LPP (Lave \& Wenger, 1991).

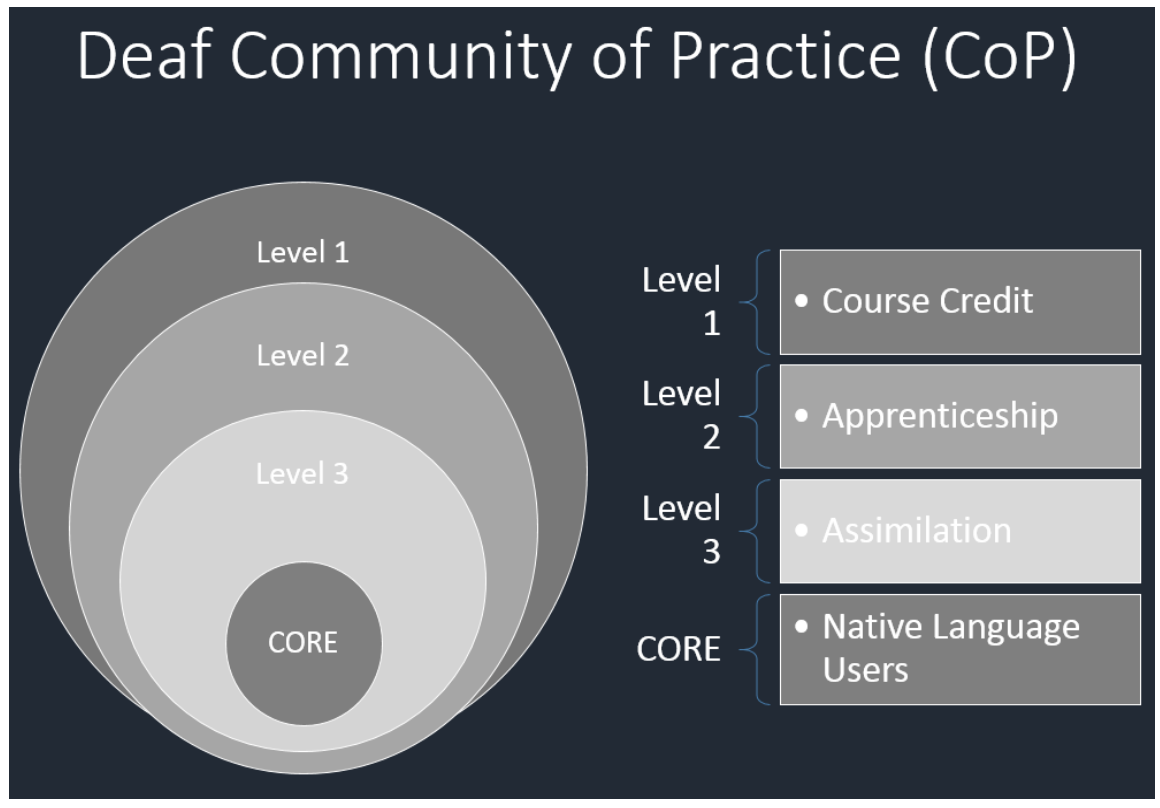

Figure 1. Deaf CoP model. This model illustrates the three levels of LPP into the Deaf community. 


\section{Significance of the Research Problem}

Rooted in the work of Vygotsky's (1978) socio-cultural theory, social interactionist theory looks at how students acquire language. Vygotsky (as cited in Schunk, 2008) thought that “... a critical component of psychological development was mastering the external process of transmitting cultural development and thinking through symbols such as language, counting, and writing" (p. 244). Exposure to native language users is essential when students are trying to learn a new language and culture. When students who are studying ASL interact with native users of the language, or other people who are fluent in ASL, they are able to develop their own skills further. The social opportunities to practice the language and interact with Deaf faculty, students, and peers, are essential for natural language acquisition (Krashen, 1988), but many students enrolled in ASL courses at WOU are unable to attend events where a critical mass of Deaf people are gathered, such as ASL Club or ASL community coffee chats. Ideally, every language learner would have the opportunity to socialize to practice their skills, but many students do not make time in their schedules to make this commitment feasible.

If students do not have the opportunity to interact with language models this might delay progress toward more advanced levels of fluency and proficiency in ASL. According to Krashen (1988), there are two types of learning that take place: acquisition and learning. Acquisition happens incidentally when surrounded by and engaged with native language users, and learning happens as a result of formal education and training in the language. Students need to have access to both types of learning to acquire a second language successfully. The incorporation of both types of learning 
(incidental/acquisition and purposeful/learning) is why it is important for the ASL Studies team at WOU to continue to evaluate the materials we use for third year ASL courses, to be sure that students are getting exposure to the information they need to help scaffold their language skills. Internal program evaluation and design is easier for instructors in the ASL Studies program to control than access to community events where students can interact with native language models. For this reason, I focus this dissertation on the attitudinal and situational barriers that impede on students' opportunities for language acquisition in Deaf CoPs.

\section{Presentation of Methods and Research Question}

During the 2017-2108 academic year, I conducted a pilot study in preparation for my dissertation research. My goal was to pilot my research questions and methods, to see if they would result in the type of data I wanted to collect for my dissertation. Based on my experience with this pilot research, and the additional information I learned in my doctorate program, I decided to modify my research questions and design. Conducting this pilot research was an effective way for me to gain valuable experience dealing with different types of data, external evaluators, and taught me the importance of continually reassessing what I want to learn from my research.

As the ASL Studies program continued to grow, and because many students continue to learn ASL with the intent to work with members of the Deaf community, I became interested in exploring the engagement of students with the Deaf community. I explored the following research questions:

1. How do students in an ASL program at a rural west coast university describe opportunities to engage with native ASL CoPs? 
2. In what ways do students learning ASL at a rural west coast university feel a part of the Deaf/native ASL CoP?

3. What experiences helped students apprentice into the Deaf community?

To address my problem of practice, I used a qualitative research design, using both descriptive and comparative research questions to complete a series of case studies involving students enrolled in ASL classes (Creswell, 2014).

I used a qualitative descriptive case study design for my research. This approach was effective for addressing my research questions. Data collected from this research helped me to identify common themes that students experience related to opportunities and barriers to interact with native ASL CoPs. The information shared by participants in this study helped inform my practice as an instructor, and understand the underlying reasons students might or might not be able to attend events, even if they know such interaction with language models may help in their language acquisition.

I used purposive sampling to select three participants for this case study research. Each participant completed a 1:1 interview with me where they shared their personal experiences, attitudes, and beliefs about participation in Deaf CoPs.

\section{Definition of Key Terms}

In this section, I provide definitions of key concepts related to my problem of practice. These terms include ASL, attitudinal barriers, situational barriers, and CoP. ASL (American Sign Language). ASL is a visual-spatial language with its own grammar, syntax, semantics, and other linguistic properties that differentiate it from English (Emmorey, 1993). Many people in the Deaf community choose to use ASL to 
communicate. The use of ASL often indicates a person ascribes to Deaf community cultural norms and practices.

Attitudinal barriers. Attitudinal barriers include the thoughts, beliefs, practices, and attitudes that cause resistance to assimilation into a CoP (National Collaborative on Workforce and Disability, 2016). In relation to hearing students learning ASL, this might be choosing to use spoken English in front of a Deaf person without signing, refusal to use ASL, or holding a belief that signed languages are inferior to spoken languages. Situational barriers. Situational barriers are the competing priorities that make it difficult for students to find time to engage with native language users in a Deaf CoP (e.g., work, family, school, income, availability of transportation, age) (Lave \& Wenger, 1991).

$\mathrm{CoP}$ (community of practice). A group of people engaging with one another for a shared purpose, goal, or desired outcome (Lave \& Wenger, 1991). 


\section{Chapter 2: Literature Review}

As more people become interested in learning about ASL and Deaf culture, CoPs within the larger Deaf community continue to grow. In this section, I provide a summary of how ASL came to exist in North America, and how it became the language of choice within the Deaf community. Research on ASL is still relatively new, with evidence to suggest that ASL is its own language appearing in the literature as recently as the 1960s. For this reason, the number of research studies that address topics similar to the focus of my dissertation research, is still small. As the field of ASL studies continues to grow, and more people become interested in this as a topic of study, more data will be available for future researchers and those interested in this field. It is my intention that my study focused on how students at a rural university apprentice into the local Deaf CoP will help to inform future practice in ASL studies.

For centuries, researchers, philosophers, medical communities, and society, believed that Deaf people were unintelligent. In 355 BC, Aristotle asserted that because Deaf people were unable to communicate using spoken languages, they did not have the ability to understand or reason and therefore had limited intelligence (Gannon, 1981). Not until $1500 \mathrm{AD}$ was this notion of limited intelligence challenged by a prominent physician named Girolamo Cardano (2018), who attested that hearing was not a requirement for understanding. Cardano contended that Deaf people could make their own decisions and possessed reasoning skills, and that despite their communication differences, they were as intelligent as their hearing counterparts (Gannon, 1981). During 
this same period, Pedro Ponce de Leon, began to teach Deaf children and adults in school type settings. He experienced great success when working with Deaf people; but unfortunately, it was not for another 260 years that others started to recognize that Deaf people had innate intelligence and could become fluent in a language if it was made accessible to them (Gannon, 1981). Once members of society afforded Deaf people with the right to use signed languages again, especially in the United States, the language and community flourished. As Deaf people had access to communication and education, they were able to successfully enter into gainful employment and be part of the larger society.

Signed Languages are complex visual-spatial languages that developed organically in communities across the world where Deaf people lived, worked, and interacted with one another (Smith et al., 2008). A common assumption is that sign language is universal, but that is incorrect. Similar to how different countries have their own spoken languages, many also have their own signed languages. Scholars can trace the origins of ASL to the early influence of a Frenchman, Charles Michel de l'Eppe, who established the first School for the Deaf in Paris (Lane, Hoffmeister, \& Bahan, 1996). What started as a school that instructed only six students quickly became a program that was responsible for teaching 60 students how to communicate using French Sign Language (LSF). During this time, l'Eppe recognized the linguistic differences between LSF and spoken French, and he documented that LSF had a complete system of grammar, semantics, and syntax separate but equal to that of spoken French (Lane et al., 1996). 
l'Eppe's first school was so successful that he opened additional schools in Rome, Amsterdam, Madrid, and Vienna. These schools became the first residential schools for the Deaf, where students lived on campus and had access to other peers and adults who could communicate with them effectively through sign language. It was in these schools that educators realized that they could teach Deaf students all subject materials using sign language. When l'Eppe died in 1789, his successor, Roch-Ambroise Cucurron Sicard continued to run the school programs for Deaf students. Two of Sicard's Deaf students, Jean Massieu and Laurent Clerc, quickly mastered LSF and became instrumental in bringing sign language to the United States (Lane et al., 1996).

Thomas Gallaudet, a minister from Hartford, Connecticut, heard about different educational approaches used to teach Deaf students in other countries. After realizing the schools in the United States were not equipped to teach Deaf students, he traveled overseas to learn more about effective educational models. He was primarily interested in learning from a school in London that taught students to use British Sign Language. The Braidwood family, who ran the school, did not allow Gallaudet to learn from their program or their approach; they refused to share any of their research or experience with him (Lane et al., 1996). Fortunately, before heading back to the United States, Gallaudet attended a lecture on LFS and met Sicard, Massieu, and Clerc. He was so impressed with their work, he asked to live with them so he could learn more about their educational approaches and study their language. His experience was so positive that he invited Massieu and Clerc to move to America with him to start a residential school for Deaf students. Massieu declined, but Clerc moved with Gallaudet and opened the Hartford 
Asylum for the Education and Instruction of the Deaf and Dumb in 1817. A few years later in 1821, it became the American School for the Deaf (ASD), which remains open as a fully functional residential school for the Deaf (Jankowski, 1997).

During the trip to America, Laurent Clerc learned to read and write English. He was already fluent in written French and LFS, and his ability to acquire written English was a testament to a Deaf person's ability to acquire language. Originally, the language of instruction at the ASD was LFS. As time passed, students started to learn written English and a new signed language emerged-ASL. ASL still borrows some signs and cognates from LFS, but scholars and educators now consider it an entirely separate language from LSF. It is also a separate language from spoken/written English, and ASL has its own grammar, syntax, semantics, and other linguistic markers (Jankowski, 1997).

Prior to the ASD opening, a local group of Deaf people lived on Martha's Vineyard Island. This Deaf community developed a form of language, communication protocols, and functioned in a larger hearing society. As documented in old journals dated from the 1500s, Native Americans had a form of signed language that existed prior to Gallaudet and Clerc opening ASD. These two contact languages likely had an influence on ASL in the early years of its development. Shortly after opening ASD, 24 additional residential schools for the Deaf opened across the United States. From 18171880, Deaf people had the opportunity to learn ASL, held positions of authority in schools and other businesses, and remained the primary educators of Deaf students. There was support for the use of ASL in residential schools and Deaf people functioned 
seamlessly as part of society (Jankowski, 1997). Additionally, Deaf people served as gatekeepers and protectors of the Deaf community, culture, and ASL.

While establishing the ASD, Thomas Gallaudet married a Deaf woman and had a Deaf son, Edward Miner Gallaudet. Thomas Gallaudet raised his son to support and advocate for Deaf rights and use of ASL. Unfortunately, while the Deaf community continued to develop their own sense of autonomy, hearing communities around the world were advocating against this development.

In 1880, many prominent Americans and Europeans were interested in the notion of eugenics (the belief and practice of promoting one race over another by controlling genetic composition), and people around the world started to talk about the promotion of a superior race. In response to the Deaf community growing in size and power, hearing educators and practitioners from around the world established a congress in Milan that addressed the use of signed languages by Deaf people (Edwards, 2007). During this congress, the leaders decided that Deaf people should be required to learn and use the native language of their country. Alexander Graham Bell (inventor of the telephone) was a prominent discourager of signed languages, and an advocate for mandating all Deaf people to use oral language. Feeling rather empowered after this congress, Alexander Graham Bell returned to the United States and was at the forefront of the movement to ban the use of ASL by the Deaf community. Edwards (2007) stated:

Bell was the most famous eugenicist specifically to attack the deaf community in his now infamous address, "Memoir Upon the Formation of a Deaf Variety of the Human Race," a paper originally delivered, to widespread acclaim in the scientific community, to the National Academy of Sciences in November 1883. Here, Bell publicly worried about the formation of a deaf variety of the human race and tried to pinpoint why such an outcome could occur. He told the gathered 
crowd that he would show that "sexual selection is at work among the deaf and dumb ... Those who believe as I do, that the production of a defective race of human beings would be a great calamity to the world, will examine carefully the causes that lead to the intermarriages of the deaf with the object of applying a remedy." (p. 90)

At the conclusion of the Milan Congress, Bell (as cited in Gannon, 1981) ordered every residential school for the Deaf in America to require students to learn to speak, read, and write English, and forbade students and educators from using ASL. According to Edwards (2007):

Though the hearing world might not remember, the Deaf world cannot forget that Bell was a staunch oralist. That is, he was a believer in and propagator of an educational philosophy that sought to ban the use of sign language in classrooms for the deaf, to conduct the education of all deaf children with speech and lipreading. More broadly, oralists attacked the use of sign language in classrooms because they wanted to secure the extinction of sign language outside the classroom. They hoped that by eliminating sign language from educational settings, by literally keeping it out of the hands of a new generation of users, they would over time succeed in driving sign language out of existence entirely. (p. 89)

This ban on sign language set in motion a long history of oppression, abuse, and a power shift away from the Deaf community. The ban on ASL devastated the Deaf community. Edward Miner Gallaudet hosted Deaf community events with the intent of saving their language. He originally proposed the use of a system called Simultaneous Communication, which required a person to speak and sign at the same time. He hoped this would appease Bell and would allow the Deaf community to continue to use some form of sign language. After learning this was still not an acceptable alternative, Edward Miner Gallaudet attended an International Congress in Paris (Jankowski, 1997) in 1900. Unlike the first congress that had only one Deaf participant, this congress has more than 200. The notion of embracing sign language easily passed because of the critical mass of Deaf people in attendance (Jankowski, 1997), but in opposition, the meeting Chairperson, 
De Lachariere, who was hearing, made a motion that Deaf people should not be allowed to vote. Hearing participants agreed with De Lachariere, and when they voted again after refusing to allow Deaf people to vote, they agreed to ban the use of all signed languages. From $1870-1890$, only $7.5 \%$ of schools for the Deaf used an oral approach to educating students, the remainder used ASL (Jankowski, 1997). After the International Congress in Paris in 1900, 80\% of residential schools no longer used ASL and followed an oral approach to educating Deaf students (Jankowski, 1997). Prior to the conference, nearly $50 \%$ of teachers were Deaf and all hearing teachers knew ASL. After the congress, only $14.5 \%$ of teachers were Deaf and those teachers were required to use oral communication (Jankowski, 1997). Communities of Deaf people continued to gather in private and use ASL because it was the most accessible language for them. This shared language, history, and culture often led Deaf people to marry one another. Bell (as cited in Lane et al., 1996) believed that if Deaf people continued to marry one another that they would have Deaf children, and so he proposed a ban that made it illegal for Deaf people to marry each other. He also mandated that Deaf students be educated in their local home school systems and not be allowed to attend one of the 24 residential schools for the Deaf. This ban on the use of ASL, marriage, forced integration, and mainstreaming, remained common practice until the 1960s (Jankowski, 1997). Decades later, schools and hearing professionals continued to advocate against the use of ASL for Deaf and Hard of Hearing individuals (Jankowski, 1997).

Researchers, medical practitioners, and others considered Deafness a low incidence minority population, and therefore Deaf people often lacked funding, access to 
advocacy resources, and support that are more prevalent in majority cultures. Until the 1960s, even though Deaf people inherently knew that ASL was a real language, they had no way of proving that to the larger community of hearing people. Not until William Stoke, a renowned linguist, proved the legitimacy of ASL, could the Deaf community begin to think about teaching others their language in a more formal, systematic way (Armstrong, 2000). Knowing about the history, oppression from majority communities, and organic nature and development of ASL in the Deaf community, is essential when trying to understand why so few resources exist in 2018 when compared to other modern languages. Recognizing how people teach ASL from person to person, in immersive environments, by fluent language models, also helps to frame the importance of access to fluent language models for students who are trying to learn ASL as a second language.

It is upon this foundational knowledge and understanding that I base the rest of this literature review. In the following section, I address research related to frameworks that affect my problem of practice.

\section{Theoretical Framework}

In this section, I explain the theoretical framework that informed my research related to my problem of practice. I base my framework on theories that have overlapping themes. None of these theories was sufficient on their own, so I intend this framework to drill down into each of the theories and to identify the most important elements as they related to second language acquisition of ASL and the impact exposure to native Deaf communities has on language learners at WOU. 
The foundation of this framework is Vygotsky's sociocultural theory (Vygotsky, 1978). In this chapter, I do not address sociocultural theory in depth because other theories that grew out of sociocultural theory are more applicable. Nevertheless, it is important to mention the sociocultural theory foundation because future researchers can consider how this theoretical framework works. One theory that grows out of sociocultural theory and connects to my problem of practice is social interactionist theory. This theory explains how students naturally acquire language (Gilardi \& Guglielmetti, 2011). A second influential theory based on sociocultural theory is social interactionalist theory. Social interactionalist theory focuses on building relationships and being in community with other language users. Both social interactionist theory and social interactionalist theory support the emersion-based ASL Studies Program offered at WOU.

A third theory that drew its original foundation from sociocultural theory is situated learning theory. Lave and Wenger (1991) developed situated learning theory, which suggests that learning happens incidentally by interaction with others in natural and authentic settings and contexts called CoPs. To take this idea one step further, Brown et al. (1989) argued that incidental learning needs to be paired with classroom instruction in what they term cognitive apprenticeship and that students should have access to both for optimal learning. CoPs are of particular interest to my current problem of practice because students cannot become fluent in the language in isolation. To learn ASL, students must engage in Deaf communities and interact with native language models. 
These language models serve as mentors and help students move from LPP to a more integrated role in the community.

Finally, the theory of second language acquisition proposed by Krashen (1982) provides a handful of key hypotheses that have further shaped my understanding of how students acquire and learn ASL as a second language. Most important to my current problem of practice are the following: acquisition learning distinction, the input hypothesis, and the affective filter hypothesis.

These four theories—-social interactionist, social interactionalist, situated learning, and second language acquisition — provide critical lenses for my research and serve as the theoretical framework underlying my examination of my problem of practice.

Social interactionist theory. Rooted in the work of Vygotsky's (1978) sociocultural theory, social interactionist theory looks at how students acquire language. Vygotsky (as cited in Schunk, 2008) believed, “. . . a critical component of psychological development was mastering the external process of transmitting cultural development and thinking through symbols such as language, counting, and writing” (p. 244). Students need to be around native language users as they start to develop their language skills. When students who are studying ASL interact with other native users of the language, or other people who are fluent in ASL, they are able to develop their own skills further. The social opportunities to practice the language and interact with Deaf faculty, students, and peers, are essential for natural language acquisition, but many students are unable to attend events where a critical mass of Deaf people are gathered, such as ASL Club or 
ASL community coffee chats. Ideally, every language learner would have the opportunity to socialize to practice their skills, but many students do not have the time in their schedules to make this commitment feasible.

Social interactionalist theory. Similar to the social interactionist theory, social interactionalist theory focuses on building relationships, interaction with peers and teachers, and involvement in activities or programs. According to Cornell-Swanson (2001), building relationships and getting involved in activities/events are key components of learning ASL. Part of learning the language includes learning about Deaf History and Deaf Culture and how they affect ASL. Students also learn about the historical role of Deaf people as gatekeepers of the Deaf community and ASL, particularly with regard to those people interested in becoming interpreters. According to Mathers and Witter-Merithew (2014):

Concern regarding the absence of Deaf heart in new practitioners is a common topic among Deaf people and seasoned interpreters ... [Historically] Deaf individuals often directly recruited individuals to serve as interpreters and invested personal time and energy guiding their acquisition and mastery of ASL, their immersion into the Deaf-World, and their induction into interpreting. (p. 159)

The opportunity to serve as gatekeepers lessened over time, as more interpreting programs hired hearing people and the selection of students became more about meeting entrance criteria, than about being invited into the community and language by a Deaf person. Mathers and Witter-Merithew continued:

Some of those who were recruited were CODAs [children of Deaf adults] and other family members. Some were individuals who worked with Deaf people in some professional capacity where their use of American Sign Language was a necessity. Others were individuals who demonstrated an interest in connecting to and communicating with Deaf people. The internal grapevine of the Deaf community was used to monitor which interpreters were most effective in 
advancing the interests of the Deaf Society and which interpreters should not be used. This is no longer the norm. (p. 159)

As more ASL courses and interpreting programs were established, the role of Deaf gatekeepers diminished. Deaf people no longer had the final say in who gained admittance into their community. According to Padden and Humphries (2005), Deaf people initially were surprised to learn that hearing people were showing an interest in learning ASL, because for so many years, Deaf people had to fight against the hearing majority for the right to use ASL. This was not an easy transition, and it led to Deaf community members questioning the intentions of hearing people who showed an interest in being part of the community. According the Mathers and Witter-Marithew (2014):

Padden and Humphries set forth several essential questions facing the Deaf community in deciding whether and with whom to share their language: "How did hearing people plan to use their knowledge of the language? Would they learn the language in order to communicate with Deaf people, or to dominate them?" (Padden \& Humphries, 2005, p. 198). Hence, the gatekeeping function points not only to language skills or communicative competence, but to the attitude and character of the outsider as well. (p. 161)

One way that educators try to ensure that hearing students learn the language (skills and competence) is by requiring students attend Deaf community events. This becomes problematic for several reasons, but most important to note is that most events happen outside of school hours so it is hard for students to make time to attend. With such a small Deaf community in Oregon, the number of Deaf events is limited. As Gilardi and Guglielmetti (2011) pointed out, finding time for these extra requirements, in addition to the hours students must spend in class during the week, can be difficult for students. In talking to colleagues across the United States, I learned that time is a common problem 
for many institutions in rural settings that offer ASL and require contact hours, but there is no common approach to address this issue to date.

Situated learning theory and CoP. According to Lave and Wenger (1991), learning happens most effectively when situated in an environment that allows for natural acquisition and not route memorization of knowledge. Historically, some communities and professions tried to meet the need for hands on learning through apprenticeship opportunities. Communities and professional originally intended for apprenticeships to be a means of teaching or acquiring a new skill, and early research on situated learning made use of this concept. As Lave and Wenger continued to explore the idea of how people learn, it became clear that there were problems with the traditional idea of apprenticeship, because it focused on repetition to master a skill, without involving other components of learning (e.g., social interaction or integration in a community). Traditionally, an environment that allows for practical application, hands-on practice, opportunities to demonstrate actively what students are learning, has been well suited for apprenticeships. Building on this idea, Lave and Wenger proposed a new theory of learning that incorporated the need for social interaction and integration called situated learning theory. Most educational systems operate counter to this idea, including our ASL Studies program at WOU, so this theory was of particular interest to me as I thought about ways to engage with students in my research. The concept of situated learning is not equivalent to simply having students engage in "hands on" activities. Situated learning also does not suggest that there should be no structure for learning, but rather that the educational approach used to guide students toward mastery is not only found in the classroom or 
during social interaction, but rather a combination of both. Lave and Wenger stated, "Learning, it seems to me, is neither wholly subjective nor fully encompassed in social interaction, and it is not constituted separately from the social world (with its own structures and meanings) of which it is part" (p. 64).

This is an important clarifying thought as I consider my problem of practice, because according to both interactionist theory and interactionalist theory, having access to native language users (Deaf people who are fluent in ASL) is more important than learning about ASL in the classroom. Lave and Wenger's (1991) assertion that mastery requires both educational and social input, lays the foundation for my dissertation research.

In situated learning, the idea of a CoP is of central importance. Lave and Wenger (1991) explained:

I propose to consider learning not as a process of socially shared cognition that results in the end in the internalization of knowledge by individuals, but as a process of becoming a member of a sustained community of practice. Developing an identity as a member of a community and becoming knowledgably skillful are part of the same process, with the former motivating, shaping, and giving meaning to the latter, which it subsumes. (p. 65)

A CoP, according to Lave and Wenger, can be any community that a person is involved in (e.g., work, school, church, sports, clubs). The community consists of members who are at different stages in their journey within the community, so at any given time people experience different levels of LPP. When a person enters into a CoP, they begin as a newcomer. Newcomers have limited knowledge about and access to the community when they first begin to engage. Old timers are people who have more experience, expertise, or who have been part of the community for longer periods. According to Lave and Wenger, 
learning happens by way of newcomers interacting and engaging with old timers. Through these relationships, old timers share knowledge, skill, and expertise with newcomers, and this allows newcomers to integrate more fully into the community. Eventually, most newcomers will become old timers, and will have opportunity to share with more newcomers. According to Lave and Wenger:

In short, investigations of situated learning focus attention on ways in which the increasing participation of newcomers in ongoing practice shapes their gradual transformation into old timers. Newcomers furnished with comprehensive goals, an initial view of the whole, improvising within the multiply structured field of mature practice with near peers and exemplars of mature practice-these are characteristic of communities of practice that reproduce themselves successfully. (p. 72)

It is important to note, however, that not all newcomers will experience the same LPP. If a newcomer is not willing to engage or is not willing to learn from an old timer, they will not continue to integrate into the community. Additionally, old timers have a lot of autonomy when it comes to deciding who becomes more central to the community and by what means.

For example, students taking ASL must attend a certain number of Deaf events each term. The Deaf community makes some events open and accessible to students, while others the Deaf community considers as Deaf space—only in rare situations will the Deaf community invite the hearing people to attend. If the first scenario, ASL students can freely engage with Deaf people at the event and can work on developing relationships within the community. The more events they attend, the more their language improves and they can communicate more fluently, the more successful they will be at becoming a more connected part of the Deaf community. Of 100 students, maybe only a handful have the right attitude, connect with a Deaf mentor, show up as allies or 
accomplices, and have exceptional language skills. This handful of students might then participate in the second scenario, Deaf space. Even if the Deaf community invited students into this additional community, they still would never become experts. They would move from newcomers to old timers, but because they are not Deaf and do not have native ASL skills, they will never be considered experts in that community. That same handful of students might be involved in another community on campus, such as ASL Club. In that community, they might serve as the experts as they interact with other students who are new to learning ASL as a second language. Therefore, LPP is a way of explaining a person's connection in a particular community. The idea of LPP within the Deaf community is central to my dissertation research (see Figure 1).

WOU is located in a geographic region of Oregon that does not have a large Deaf community. There are not a lot of ongoing Deaf events that ASL students can attend to engage with a native ASL CoP. This makes it difficult for students to integrate into the community and move from their original role as a newcomer. Several questions come to mind when considering how to offer students with the most effective opportunities to engage with the Deaf community. For example, what type of community events provide enough opportunity for students to engage in the community and feel more connected? How will the local Deaf community feel about engaging in more events with the goal of mentoring students in hopes of creating a stronger community of professionals and allies? What do students need to feel more comfortable in order to engage in community events? How might Deaf people welcome more second language learners into the community if 
they felt that students were not only attending because of ASL course requirements? All of these questions play into my problem of practice.

In their research about CoPs, Lave and Wenger (1991) stressed the importance of history, culture, and social engagement. These same three qualifiers are parallel in Deaf culture, which makes this theory easy to apply to second language learners of ASL. As noted earlier, the Deaf community has faced a long-standing history of oppression at the hands of hearing people. Professionals, steeped in practices of audism, tried to fix our community by forbidding us to sign and forcing us to try to learn to speak and read lips. This denied our community opportunity to develop a shared culture and language for many years, and served as a means to deconstruct many of the Deaf CoPs that were in existence prior to this shift in language philosophy (Jankowski, 1997). Deaf people are resilient and those who fought for linguistic access formed their own community, made up of only other Deaf people who supported the use of ASL and who believed in the value of Deaf culture. Nearly 140 years later, the Deaf community continues to fight for its rights to be part of society and respected for its language. In this way, the Deaf community remains in flux as old timers pass away and newcomers enter our community without a shared understanding of the oppression and discrimination our community faced over the years.

Something I continue to be mindful of is that the Deaf community is a minority group. When asking Deaf people to welcome hearing people into the community, it is a conscious request to allow members of the majority group into their sacred and safe space. I referenced Deaf space and the notion of only hand-selected members from the 
hearing community given entrance into that inner circle of the Deaf community. Still, the most central, internal community within the larger Deaf community is reserved for those who are culturally and linguistically Deaf. This space, no matter how fluent a second language user becomes; it is a space always reserved and held sacred as Deaf space.

Students who learn ASL as a second language have their first CoP experience in the classroom with their teacher and classmates. This is one reason that it is critical for ASL teachers to be Deaf, because only then can students experience their first steps into the Deaf community. Students must suspend their own affiliation with the majority community (hearing community), and assimilate with Deaf community norms in immersion-based classrooms. Students learn to drop English form, not use their voice in the presence of a Deaf person, and to adhere to other cultural values that are import to the Deaf community. Students then have the opportunity to build connections in the larger Deaf community, and their LPP in the larger Deaf CoP will depend on their attitude and willingness to respect the culture and participate in social engagement.

Critique of situated learning theory. According to Anderson, Reder, and Simon (1996), some of the claims made by Lave and Wenger (1991) related to situated learning theory could be inaccurate. They take exception to four specific claims. First, they disagreed with the notion that learning must take place in a specific environment for students to learn how to apply what is learned. They stated that while Lave and Wenger suggested that learning happens in applied settings (e.g., a person learns how to count change at the store but is unable to compute similar math equations on a math exam) 
differently than in a classroom, that what is learned in the classroom can often be applied in various settings.

Based on their research, Anderson et al. (1996) disagreed with a second idea that knowledge does not transfer between similar types of distinct skills. While Lave and Wenger (1991) stated there is little to no research that suggests that learning transfer is effective, Anderson et al. provided numerous examples of studies that address the transfer of knowledge and skill beginning almost 200 years ago.

Third, Lave and Wenger (1991) argued against the idea of successful abstraction of knowledge learned in schools when trying to apply that knowledge in the real world. Anderson et al. (1996) stated that abstract instruction could be effective if done in a wellplanned and meaningful way. They reported a need for a combination of concrete instruction and abstract instruction for real learning to occur that can be applied on the job.

Finally, Anderson et al. (1996) disagreed with the idea that all learning needs to happen in environments. Lave and Wenger's (1991) research supported learning while interacting in the community where the skill or knowledge applied. Anderson, et al. suggested that not all skills or knowledge learned while engaging with the community because this could cause cognitive overload. They asserted that people learn some tasks (e.g., learning tax codes) independently, outside of interaction with consumers who will benefit from your newly learned skill. 
Overall, while Anderson et al. (1996) disagreed with some points of situated learning based on their own experience and research, they did not present any substantial information that deters me from using this theory in my practice.

Second language acquisition theory. Learning a second language in the United States is often a very different experience than learning a second language in many other countries. In most European countries, children between the ages of six and nine years old are required to learn a foreign language (Devlin, 2015). According to Devlin (2015), there is no national foreign language mandate in the United States. Often, states give individual school districts and postsecondary institutions the power to determine what foreign language requirements they want to enforce. In the United States, schools do not specify language offerings or language requirements other than English. ASL is the fourth most studied language in the United States with nearly 110,000 students enrolled, according to the Modern Language Association's 2013 report (Goldberg et al., 2015). Even with such a large number of students enrolled in ASL courses, research on ASL is still in its infancy, due in large part to the hundred years of oralism with its intent to eradicate ASL. As the Deaf community continues to fight for rights to an accessible language, more research is emerging that will likely influence the way that instructors teach ASL in the future. As of 2018, the number of curricular materials available for teachers who teach ASL as a second/modern language remains very small. As more programs begin to require ASL fluency (e.g., teachers of the Deaf, rehabilitation counselors for the Deaf, interpreting) hopefully more focus will be placed on the evaluation of current curricular materials and the development of new materials. 
Acquiring a second language can be a complicated process. According the Krashen's (1982) second language acquisition theory, five key hypotheses need consideration for second language acquisition: the acquisition-learning distinction, natural order hypothesis, the monitor hypothesis, the input hypothesis, and the affective filter hypothesis.

In the first hypothesis, the acquisition-learning distinction, Krashen (1982) stated that there are two separate types of processes involved as a student studies a second language. The first is acquisition, and that is comparable to how children acquire their first language through exposure to the language, incidental learning, and access to native language users. The second is learning, and that is when someone receives formal instruction on components of the language (e.g., vocabulary, grammar).

In the second hypothesis, the natural order hypothesis, there is a natural progression to how most people acquire the grammar of a language. Interestingly, that order/progression is different for first language acquisition versus second language acquisition. The components of language that people pick up first if the language is their first language are not the same as the components that are picked up first if it is their second language (Krashen, 1982).

The third hypothesis, the monitor hypothesis, centers on the acquisition-learning distinction hypothesis. This third hypothesis also examines the roles of acquisition and learning, but from a slightly different perspective. According to Krashen (1982):

Normally, acquisition "initiates" our utterances in a second language and is responsible for our fluency. Learning has only one function, and that is as a Monitor, or editor. Learning comes into play only to make changes in the form of 
our utterance, after is has been "produced" by the acquired system. This can happen before we speak or write, or after (self-correction). (p. 15)

In this way, it seems that the monitor uses what students learned in the classroom (formal instruction) to make repairs, instead of using formally learning to express the utterance.

The fourth hypothesis, the input hypothesis, contradicts most other second language acquisition theories that assume that a person must first memorize vocabulary, grammar, and rules, and then learn to apply them in conversation. Instead, in his input hypothesis, Krashen (1982) asserted that through acquisition of language from other native language users, a person attempts to reciprocate that language, and in those attempts to create communication, they acquire the structure/grammar of the language. He noted that the input hypothesis only applies to acquisition (not learning), and that the level of language the student needs to be surrounded by should always be at a slightly higher level than their current skill level/competence. Additionally, Krashen stated, “. . speaking fluency cannot be taught directly. Rather, it 'emerges' over time, on its own. The best way, and perhaps the only way, to teach speaking, according to this view, is simply to provide comprehensible input" (p. 22). This is a significant point because it suggests that without access to a native language user, acquiring a second language will not be possible. While Krashen used the term "speak," this concept applies to ASL because the point is that the input (e.g., speech, sign) must be high quality.

Krashen's (1982) fourth hypothesis was fundamental to the research I conducted with ASL students at WOU. These were students learning ASL as a second/modern language, and according to this hypothesis, the key to fluency is interaction with native language users over time. Many students who take ASL for the first time often report that 
they think ASL will be easy to learn (Kemp, 1998). Later, they realize that ASL is not a form of gestural communication, but rather a language, complete with its own grammar, syntax, and other linguistic and sociolinguistic features. The implication of this hypothesis points to a critical issue with how we teach ASL within the university system. At WOU, each term is 10 weeks in duration, and students spend 4 hours per week in each ASL class, for a total of 40 hours of direct formal instruction (learning). Students are required to attend WOU's ASL Club, and one or two Deaf events each term, but there are no time requirements or official ways that instructors are able to monitor if students actually engaged in conversation with Deaf community members while at events.

According to the input hypothesis, if fluency cannot be taught, and must be acquired through interaction with quality inputs (Deaf language models), then ideally students would spend the majority of their time interacting with other native language users, and a smaller portion of their time in the classroom. Due to the university system, I was unable to decrease the amount of classroom hours students were required to attend during participation in my research, but this hypothesis did support my research design that required students to commit to attending as many Deaf and ASL events as possible during the course of the study.

An additional consideration is that Deaf ASL users often engage in code switching when communicating with second language learners. For example, if a Deaf person recognizes that a student is just learning ASL, they know immediately to change their register and adjust their output so students can understand what they are saying (Quinto-Pozos \& Mehta, 2010; Wilcox \& Wilcox, 1997). While I always assumed that 
this was positive, because it made students feel more comfortable and seemed to increase comprehension, according to Krashen's (1982) input hypothesis, the input should be slightly higher than the level of competence, so maybe code shifting to make it easier for students to understand actually is to their detriment. This was an important consideration as I thought about the types of interactions within the Deaf CoP that I requested research participants to attend.

In his fifth hypothesis, the affective filter hypothesis, Krashen (1982) described the role of affect and acceptance of input and feedback in second language acquisition. This is one of the biggest barriers instructors face when working with ASL students at WOU. According to Krashen:

The Affective Filter hypothesis captures the relationship between affective variables and the process of second language acquisition by positing that acquirers vary with respect to the strength or level of their Affective Filters. Those whose attitudes are not optimal for second language acquisition will not only tend to seek less input, but they will also have a high or strong Affective Filter-even if they understand the message, the input will not reach the part of the brain responsible for language acquisition, or the language acquisition device. Those with attitudes more conducive to second language acquisition will not only seek and obtain more input, they will also have a lower or weaker filter. (p. 31)

This hypothesis is consistent with what I often see in students who take ASL to fulfil the modern language requirement, as opposed to taking ASL because they plan to use it in a future profession. According to Jacobowitz (2005), on average it takes 10 years for a person to become fluent in ASL, and during that time there is some indication that some students become discouraged with feedback and input from instructors, causing them to activate more affective filters.

Krashen (1982) stated that it is important to create situations that perpetuate low affective filters. WOU students tend to be less nervous when attending ASL club on 
campus, but there are not usually any Deaf community members who attend ASL club. For the most part, this is a time set aside each week for students to practice ASL in an immersive environment with their peers, but without fluent input from Deaf language models, this is not an effective use of students' time. One key question posed during interviews with participants was what types of situations, settings, or environments they felt would cause them to have a higher filter, as opposed to those that would encourage a lower filter. I know that the answer differed for each student, but it was interesting and informative to collect feedback and see if there were any commonalities across participants.

Krashen's (1982) first three hypotheses are important and foundational, but the fourth and fifth hypotheses clearly identify the biggest obstacles I encounter with students at WOU. According to Krashen, there are specific:

... factors that have been thought to be related to second language acquisition success, including instruction, different measures of exposure to the second language, and the age of the acquirer. These factors, it will be claimed, are not really causative factors. While they seem to relate to success or failure to acquire second languages, the true causative variables in second language acquisition derive from the input hypothesis and the affective filter - the amount of comprehensible input the acquirer receives and understands, and the strength of the affective filter, or the degree to which the acquirer is "open" to the input. (p. 9)

Knowing that quality input and affective filters are so important, I was able to better plan for meaningful induction into the Deaf CoP for participants in my study. Recognizing that all students would enter with a different filter, and a different level of competence, I figured out how to ensure that students would have access to Deaf fluent language models who would be able to help them lower their filter while still engaging them at a level slightly higher than their current level of competence $(i+1)$. 
Critique of second language acquisition theory. A quick search of the literature yields many critiques of Krashen's (1982) work over the years. Most articles I read pointed to authors who first took offense to his claims. Rather than listing all of these authors, because there were many, I selected the most comprehensive article that I found that seemed to identify the most common critiques regarding Krashen's Input Hypothesis. Liu (2015) began by acknowledging that Krashen has made substantial contributions to the field of second language acquisition. Through his research of the literature, he identified common themes across all of Krashen's work. These same themes were consistent with critiques specifically targeted at Krashen’s Input Hypothesis.

According to Liu (2015), there are three major critiques (arguments) relative to the Input Hypothesis: vagueness, oversimplification of input, and overclaims. In terms of the input hypothesis, Krashen's (1982) major assertion was students need to have access to "comprehensible input" to acquire a second language. Krashen explained why this is important, and contrasts this input with formal instruction found in a classroom, but he never specifically defines what he means by comprehensible input. This vagueness, whether intentional or not, leaves the reader to define the meaning of comprehensible input on their own. This can lead to very different interpretations depending on a person's personal or theoretical perspective. This can be problematic when the most foundational element of the hypothesis leaves that much room for personal interpretation.

The next issue cited in the literature is related to Krashen's (1982) explanation that comprehensible input is similar to caretaker speech (Liu, 2015). While Krashen never clearly defined what comprehensible input is, he likened it to other types of 
communication, like caretaker speech or motherese, that altered to fit the level of the person who is participating in the conversation. Liu pointed to many people who disagree with this point (Gregg, 1984; McLaughlin, 1987; White, 1987) and suggested that caretaker speech is not always simplified, and that based on other studies done that measure language acquisition, specifically L1 acquisition, children often do pick up more sophisticated language that they are exposed to, which contradicts the need to simply the input.

The final issue that Liu (2015) addressed is Krashen's (1982) claim that the input hypothesis is the most important concept in second language acquisition theory. Liu pointed to the fact that while input hypothesis is important, it is not the most important, or the only important factor to consider when examining second language acquisition.

Despite the people who take fault with Krashen's (1982) theories and hypotheses, none of the critiques I read seemed to be sufficient reason to abandon second language acquisition theory as a fundamental part of my theoretical framework for this dissertation research. In fact, the issues of vagueness, simplification of input, and overclaims are not with the focus of my research because these are not issues that often see encounter in our ASL Studies program.

\section{Review of the Research Literature}

As ASL is still a relatively new language, there is limited research available that addresses topics similar to my problem of practice. This lack of research does not indicate that the problem does not exist, but rather points to how society has marginalized and overlooked the Deaf community and its language. Due to limited resources and 
funding, Deaf people have not historically been in positions of power that enable them to design and run research studies, or to participate in research about ASL. As more Deaf people have access to quality education and persist through levels of higher education, hopefully more research will delve into the core of teaching ASL as a second language, and how this process affects both Deaf and hearing communities. Still, there remains a lack of tenure track positions at universities where research is valued, and this creates missed opportunities for Deaf people to be part of the research process.

In a recent presentation at the Conference of Interpreter Trainers, Holcomb (2018), a renowned Deaf leader and educator, recently lamented this fact during a national conference where he shared that because he works for a community college, he has no ability to seek out research opportunities to push the field of ASL instruction forward. His campus does not have the capacity to do research, has no IRB process, and there are no grants that focus on research. This leaves him without the opportunity to contribute to the field via formal research, but his argument was that Deaf people around the world continue to do informal research and evaluation every day as they teach, talk with other educators, modify their instruction, and try again (Holcomb, 2018). This type of research is not valued in the same way as university-based research, which means a disproportionate amount of research happening at the grassroots level by Deaf educators goes without recognition or dissemination. With only a limited number of positions for Deaf educators, this lack of research will not be a fast or easy fix. That said, two studies addressed topics similar to the one I am explored in my research. 
California State University, at Northridge, (CSUN) has a long-standing history of being inclusive of Deaf students and faculty on their campus and in their programs (Morgan, 2014). For many years, the Deaf community considered CSUN as one of the most accessible options for Deaf students who are interested in attending college. For this reason, a critical mass of Deaf community members attend school, work on campus, or live in the surrounding community. In a descriptive study by McKee and McKee (1992), a total of 12 ASL instructors and 72 ASL students participated in a survey meant to explore perceived difficultly in learning ASL as a foreign language. Students selected to participate in this study were in upper level ASL courses (ASL 3, 4, or 5) and most were in pursuit of a Bachelor of Arts degree in Deaf Studies. Instructors were fluent in ASL and almost all had more than 14 years of teaching experience (McKee \& McKee, 1992). The study consisted of a slightly different design for instructors and students.

Some instructors participated in only the written survey, and others participated in the written survey and a follow-up interview. There were 12 instructors (six Deaf, six hearing) who completed the written survey. McKee and McKee (1992) then selected four of the instructors (2 Deaf, 2 hearing) for follow-up interviews. All responses from the surveys and the interviews were included in the results of this study. The process of creating the final survey for students occurred in two parts. The first part consisted of an open-ended questionnaire administered to 39 students. The researchers used answers from this questionnaire to create the closed questionnaire/survey given to 72 students to complete (McKee \& McKee, 1992). 
McKee and McKee (1992) found that instructors and students talked about both linguistic and affective difficulties in learning ASL. As the focus of my dissertation research relates to how students engage and apprentice in local Deaf CoPs, I focused on the affective portion of McKee and McKee's results. They found that even with a large Deaf population and many people using ASL on and around campus, hearing students who enrolled in ASL courses at CSUN still reported facing barriers to language acquisition. In their study, students reported facing barriers to increasing their ASL fluency. In general, students found it difficult to find time to attend events and practice their ASL skills. I found this interesting because with a larger number of Deaf events available, my assumption would be that students would have more options and could find events that fit into their schedules more readily, but in reality, students still reported that they were unable to make it to events. This is an example of what I refer to as a situational barrier students at WOU often describe, but even with increased opportunities, students at CSUN still faced similar experiences.

Students reported feeling as if their linguistic skills were not good enough to interact with Deaf people outside of their classroom environment (McKee \& McKee, 1992). They also stated feeling frustrated because some native ASL users switched to more English-like signing when interacting with them, or purposefully slowed down their communication because they thought the student would not understand. This made students feel a lack of confidence in their language fluency. Students also mentioned not being used to the amount of required eye contact (a must in ASL), being shy in front of new people, and feeling nervous to use their ASL skills outside of the classroom (McKee 
\& McKee, 1992). All of these remarks are similar to ones that WOU students expressed anecdotally, and it is interesting to note that McKee and McKee conducted their study in 1992; as of 2018, these same attitudes, thoughts, and beliefs still prevail.

A similar study conducted by Yang and Kim (2011) focused on second language learner beliefs and the ways those beliefs affected their study aboard experiences. This study was not about ASL, but the basic premise is the same. This study consisted of four students, but the authors only examined the stories of two students in the article. Yang and Kim's study was rooted in Vygotsky's sociocultural theory. Rather than adopting a traditional lens of how students acquire new knowledge by slowing adding to their knowledge base, they encouraged the process of remediation, which requires individuals to examine their own experiences and interactions in addition to the content they are learning. In this case, Yang and Kim used a qualitative approach to examine student's beliefs and attitudes about their language acquisition and their study abroad experience. To ensure good data triangulation, participants engaged in various forms of data collection (e.g., pre and post interviews, monthly journal entries, and language recall tests). At the conclusion of the study, Yang and Kim found that students' beliefs and attitudes must align with their learning experience in order to be successful. In this case, both students originally thought a study aboard experience would help them acquire a second language more effectively. The student who participated in a study aboard experience but who did not align his beliefs and attitudes with that experience did not notice significant improvement in his language acquisition. He left the program believing that study aboard was not as effective as he had anticipated. By contrast, the student who 
participated in a study aboard experience and when met with obstacles, was willing to change her attitude and beliefs about language acquisition and the experience of study aboard, felt her experience was effective.

Similarly, students learning ASL face barriers apprenticing into Deaf CoPs; Deaf professionals often face barriers that influence their professional lives. Houston (2018) conducted a mixed methods study that included a survey $(n=55)$ and phenomenological interviewing $(n=8)$, that focused on the experience of Deaf faculty members at five institutions of higher education across the United States. Houston's main interest was exploring why Deaf educators in higher education ended up teaching ASL rather than teaching classes in their own fields of study. Houston identified four main themes from her analyses: systemic factors, difference, elitism, and success and barriers.

Participants stated they often felt oppressed because their administration operated from an audist mentality, and people did not understand the differences between hearing and Deaf communities. An example Houston (2018) cited related to interpreter hiring practices through the human resources department at colleges and universities. Hiring unqualified interpreters (those who do not have language fluency) causes frustration and lack of access for Deaf educators, and can have an adverse impact on their professional lives. When administration is unaware of the importance of hiring qualified providers, rather than "warm bodies," Deaf educators suffer the consequences. This finding has implications for WOU's ASL Studies department, because as students complete their ASL coursework and move on to professional fields that require the use of ASL, it is 
important these students have fluent language skills. When they do not have fluent language skills, this directly affects Deaf professionals as found in Houston's study.

Another theme from Houston's (2018) research was elitism. Interestingly, though this topic of elitism in the Deaf community and professional field came up in her findings, participants did not elaborate on why these stated this was a common barrier for them related to employment. The concept of elitism in the Deaf community is not new or isolated to this study. In general, those Deaf individuals who have Deaf family members (multi-generation) most commonly are referred to as elite because they had access to fluent ASL models from birth. Whether this elitism actually has far-reaching impact on Deaf professionals and their ability or opportunity to teach in their preferred fields of study, remains uncertain, because participants were so reluctant to provide detailed information for fear of the researcher being able to identify the professionals they were describing. The Deaf community, especially those who work in higher education, is so small, that participants took extra precautions in their responses, which led to inconclusive findings in this area.

In general, Houston (2018) found that when Deaf educators had the appropriate supports in place (e.g., interpreters, support systems, working relationships with coworkers) they reported feeling more able to succeed in their work environments. Many noted that the addition of diversity training to hearing colleagues and administration (e.g., human resources) would be beneficial and have an impact on Deaf professionals' experiences in higher education environments. 
Both McKee and McKee (1992) and Houston's (2018) studies helped inform my thinking and pave the way for my research. McKee and McKee looked at a similar problem of practice, but one situated in a large Deaf community more than 25 years ago. It was fascinating to note that not much has changed from the time McKee and McKee conducted their study in 1992 to 2018 -19 - the year I conducted my study. I anticipated finding similar results to McKee and McKee, but I was curious about differences based on location of our university (rural vs. urban), and because WOU has so many graduate programs that require ASL fluency for admittance.

Houston's (2018) study focused on the opposite end of the spectrum — rather than on hearing people learning ASL, the focus was on how Deaf people are impacted by professionals who are not fluent in ASL (e.g., interpreters, administration, colleagues). This study gives credence to the concerns we have at WOU in the ASL Studies Department that students' fluency will eventually have an impact on Deaf professionals, particularly the Deaf instructors who work at WOU.

Similar to how it is important for students to apprentice into a Deaf CoP as they learn ASL, it is equally as important for Deaf professionals to have their own CoP for engagement and learning. While not directly related to the Deaf community and ASL, Hodkinson and Hodkinson (2004) conducted a qualitative case study of two participants who were teachers in a secondary school in England. This study focused on the dispositions and work habits of the two teachers. Both teachers were exceptional educators and they shared many of the same basic characteristics (e.g., race, age, status). Through a series of semi-structured interviews with the two participants, additional 
interviews and interactions with other teachers and staff, and through observation of each teacher's instructional approaches, Hodkinson and Hodkinson determined that many variables contributed to the success of each teacher. Their approaches to teaching and learning were very different from one another, but they both still encountered personal success by using their own philosophical and instructional approaches. In addition to general disposition and style of learning, CoPs played a role in the success of each teacher. One teacher worked with a mentor and received valuable modeling and feedback, while the other teacher preferred to take a leadership role and instruct others instead of learning from them. At the conclusion of the study, Hodkinson and Hodkinson (2004) determined that it was not possible to identify one specific factor that made both teachers successful, but rather that it was a combination of many factors (e.g., learning style, teaching style, $\mathrm{CoP}$ ) that played a role in their success. For Deaf professionals, the opportunity to be part of a CoP that includes other Deaf individuals at their place of employment might be a challenge.

At stated earlier, little research exists on my current problem of practice; however, Houston (2018) and McKee and McKee (1992) were able to identify important information that can be used when considering how to educate students learning ASL. Their findings also have implications for how students' skills ultimately have an impact on Deaf professionals who rely on them for access to communication.

As stated earlier in this chapter, research related to students' perceptions about learning ASL and apprenticing into a Deaf CoP is very limited. After a thorough review of the literature and reaching out to colleagues who are also conducting research in 
doctorate programs, I decided to broaden my review to include other language acquisition. As seen throughout this dissertation, research done in other areas may be applicable to the study of ASL, but there are some unique challenges that exist in only the Deaf community because in addition to using a minority language, we are a minority culture. It is my hope, and the consensus of the Deaf community, that additional research needs to focus on these topics by Deaf scholars in the very near future.

\section{Review of Methodological Literature}

To address my problem of practice, I used a qualitative research design, including both descriptive and comparative research questions to complete three case studies (Creswell, 2014). According to Yin (2018), case study research includes five components of research design: (a) questions, (b) prepositions, (c) defining the case/boundaries, (d) linking data and prepositions, and (e) criteria for interpretation. If any of these five components are absent, the researcher might not get the information needed to address their research questions. Generally, a case study is a good fit when answering "how" and "why" based questions. After identifying the research question, the researcher needs to make some prepositions. These prepositions direct the researcher's attention to specific areas of their research they want to focus on. Once a researcher has developed some prepositions, they need to define the case(s) to be included in their study. Researchers may choose to select one case or perform a multiple-case study, and they must clearly define participant boundaries (who is included and who is not). Developing good prepositions will also help researchers ensure they build in specific elements into their research (e.g., time, location) so that when it is time to interpret their data, they can use 
these elements as part of their analysis (Yin, 2018). Finally, the researcher must set up criteria for evaluation and interpretation.

The use of case studies as a teaching tool has been in practice for many years. However, according to Yin (2018), not until recently have researchers accepted case study design as a method of research — instead of just an exploratory measure embedded in another type of research method. Yin clarified,

. . . case study research comprises an all-encompassing mode of inquiry, with its own logic of design, data collection techniques, and specific approaches to data analysis . . . case studies are not limited to being a data collection tactic alone or even a design feature alone. (p. 16).

Yin (2018) explained that there are some critiques/concerns about case study research. The first concern questions the rigor of case studies. If the researcher does not have a clear plan, does not adhere to correct procedures, or applies their own bias when interpreting results from the case studies, this is problematic. Second, there are different types of case studies, and not all involve research. Specifically, there are three types of "nonresearch" case studies: teaching-practice case studies, popular case studies, and case records (Yin, 2018). These types of case studies do not adhere to research standards or practices; they are not the same as research-based case studies. It is critical to understand the difference between research and nonresearch case studies. A third concern about case studies relates to the generalizability of the results collected from the study. This type of research can lead to generalizable theories, but not statistical generalizations (Yin, 2018). A fourth concern relates to the amount of time and effort involved in case study research. As new ways to conduct case study research become clearer, this may become less of a concern. It is important that the researcher have a clear understanding of the type and 
amount of data that needs to be collected, and adheres to their design model when collecting data. This will keep the amount of time and work, in addition to the amount of data collected, more reasonable. A final concern Yin (2018) shared relates to why a researcher would choose a case study design rather than an experimental design. Whether or not case study data, or experimental data, is best fit depends on the goal of the research, and the research questions the researcher is trying to answer.

For my specific case study research, I conducted interviews to gather qualitative data. According to Seidman (2013), interviewing is not a way to test a hypothesis or do evaluation. Instead, interviewing is for collecting the stories and experiences of a specific group of identified individuals around a common phenomenon. There are various ways to conduct interviews, and they range from very structured (specific closed ended questions) to unstructured (all open-ended questions). Interviewing can be very labor intensive and time consuming, and the results can sometimes be difficult to analyze if the researcher is not clear and consistent on how they decide to interpret data. The overarching goal of interviewing is to analyze individual stories, compare those with other stories, and see if the researcher identifies enough patterns that form a new theory. Knapik's (2006) study is a strong example of a research study that used interviewing effectively.

Knapik's (2006) research examined four participants' experiences through interviews as a part of a qualitative research study. According to Knapik, early research findings about the use of interviews and their impact on participants, has led to a carefully prescribed way that most researchers conduct interviews. She asserted that this approach might not be the most effective for all participants. Knapik found that 
participants in her study favored an approach that allowed for engagement between the interviewer and interviewee (two-way dialogue and experience sharing). They also favored an environment that allowed for open conversation around a specific phenomenon. Participants shared that these approaches made the interaction feel more natural and like both participants and the interviewer had a shared interest in the topic and goal in discussing it. The approach that was least liked by all four participants was the one-way dialogue, which is the most common approach, because they felt that they were simply responding to questions with no opportunity for interaction or interplay with the interviewer. Knapik suggested that a combination of approaches would be effective when trying to collect complete responses from interviewees.

Knapik's (2006) exploration around the types of interviews and participants' preferences, made me think about my interactions with my participants. I planned to interview participants about their experience, attitudes, and beliefs; however, I also wanted to know about their language acquisition over the course of one academic year. Knowing that I can mix interview approaches seemed more feasible. I also appreciated that Knapik stance that a researcher needs to be flexible and meet the needs of the interviewee. With my personality, I like flexibility and being able to change things if I notice they are not effective. Within my study, I wanted to make sure that participants felt safe and supported so that I could get the information I needed from the interaction.

Pilot study. I think it is important to disclose that I conducted a pilot study related to my research topic during the 2017-2018 academic year. I received funding to evaluate students' ASL skill levels before and after completing ASL 7, 8, and 9. This project was 
meant to prepare me for my dissertation research; however, rather than confirming my intention to focus on measuring students' skills, it actually made me realize that I did not want to focus on skill acquisition. Instead, I focused my attention on the shift in attitude and belief as students assimilate into a Deaf CoP.

Nevertheless, the data I collected were valuable because they gave the WOU ASL Studies team a better idea of the skill level of students who take ASL 7-9. We have often changed our curriculum and our pedagogical approaches to how we teach these specific courses, and that has left us wondering about what students actually learn during the third year of ASL. To measure the change in skill level for students taking this course sequence (ASL 7-9), students participated in pre and post Sign Language Proficiency Interview assessments, proctored by raters employed by the National Technical Institute for the Deaf.

The original design accounted for 16 students, eight traditional and eight nontraditional, but due to extenuating circumstances, only 10 students completed the SLPI assessment prior to beginning ASL 7 fall term of 2017. The SLPI coordinator had a difficult time getting students to commit to video interviews and even with a local proctor to coordinate emails and schedules, we were unable to get all the original students who signed up to complete their interview in a timely manner. The same students were asked to complete a post interview after completing ASL 9, but only eight students participated in this interview assessment spring term of 2018. In an attempt to remain as impartial as possible, I did not know which students participated in the research study until after they 
completed both pre and post interview assessments because some of them took ASL 7-9 with me this academic year. Later, I received the summary of results from the SLPI team. Between fall 2017-spring 2018, three participants significantly improved their ASL fluency skills as evidenced by scoring in a higher diagnostic category on the SLPI interview assessment. Two students moved from intermediate to intermediate plus, and one student moved from intermediate to advanced category. Five participants retained the same level of fluency from fall 2017-spring 2018. I was not sure how to feel about these results because I was hoping to see major improvements across all students. However, when I consulted with the SLPI administrator, he said that once students near the advanced level, measurable changes within one academic term are uncommon. This was good information for me to know because otherwise I might have felt as if we were failing our students because they were not making significant progress during their third year of ASL course work.

I think many factors played into students' fluency, such as length of classes (term vs. semester), the size of the local Deaf community, number of Deaf events, the number of contact hours, or other variables that we are unsure of how to account for. It is worthwhile to continue to research the progress students make in upper division ASL courses, although this was not be the focus of my dissertation research. I remain more curious about how people come to feel accepted and embraced by the local Deaf CoP as opposed to fluency levels of students who complete our program. This shift in focus, to get to the heart of why a person feels they are able to engage with and be an active 
community member of, a Deaf CoP, helped solidify the use of case study design as my research methodology. 


\section{Chapter 3: Methodology}

WOU holds an exemplary reputation for its four-year ASL program. Students who continue on to the third and fourth year of ASL coursework often intend to continue their education or find employment in a Deaf-related field. One common barrier that continued to surface for students was limited meaningful interaction with a local and native Deaf/ASL CoP. The purpose of this study was to explore attitudinal and situational barriers that make it difficult for students to move past LPP in the Deaf community (Lave \& Wenger, 1991).

\section{Research Questions}

The following were the research questions that guided my dissertation project:

1. How do students in an ASL program at a rural west coast university describe opportunities to engaging with native ASL CoPs?

2. In what ways do students learning ASL at a rural west coast university feel a part of the Deaf/native ASL CoP?

3. What experiences helped students apprentice into the Deaf community?

\section{Research Methods}

To address my problem of practice, I used a qualitative research design with both descriptive and comparative research questions to complete three case studies (Creswell, 2014). This was an effective method to use to address my research questions because I was able to collect personal stories from individuals related to their experiences, frustrations, successes, and failures as they tried become more connected to the local Deaf community. 
According to Merriam and Tisdell (2016), qualitative research requires the completion of two different types of analysis. The first type of analysis focuses on the individual case. For this reason, I performed this type of analysis for every case I was studying (each individual student). The second type of analysis requires the researcher to look for patterns and compare findings between all of the cases (across students). Therefore, I conducted an analysis across all of my cases. While this provided me with good information, according to Merriam and Tisdell, the amount of data collected in this approach can be overwhelming and requires good organization, categorization, and coding, which I managed using online software: Smartsheets and GoReact.

Merriam and Tisdell (2016) described approaches to categorization and coding of data. As I developed the interview questions, I identified an initial set of codes based on years of experience teaching ASL and recognized common barriers that impede students' language acquisition. Possible codes included work, family, money, and transportation. These seemed to be the most common competing priorities for students, so while I had them on my radar and I was careful not to ask leading questions that encourage students to answer in any particular way when conducting the interviews. According to Merriam and Tisdell, researchers can use additional type of coding called analytical coding. Using this type of coding, I needed to group items together that made sense to combine. For example, if a student said they do not have a car, the bus does not run from Monmouth to Corvallis, or that it is too cold to ride their bike to events in the winter, I could code these examples as lack of transportation. I needed to code each case study individually, and then looked at the codes across all of the case studies and compared them to get my 
results. This coding process allowed me to analyze my data across all case studies and begin to develop possible explanations of what factors affect a student's fluency rate, and/or what competing priorities might make it difficult for a student to improve their fluency.

Participants. To qualify for this study, participants needed to be students enrolled in ASL 302 during the winter 2019 academic term and must have completed all previous ASL coursework in good standing. Students also needed to plan to complete ASL 302 and 303 during the 2019 academic year. My goal was to have three students participate: one student who never took ASL until enrolling at WOU, one student who took ASL at a community college or another university, and one student who had exposure to ASL in high school. The three participants needed to express an interest in becoming a more meaningful part of the Deaf community.

Procedures. My data collection and analysis occurred between January-May of 2019. During the beginning of winter 2019 term, I announced the opportunity to participate in this study to all three ASL 302 classes ( 65 students) and provided a form for students to fill out if they were interested in participating (see Appendix A). This form prompted students to provide basic demographic information and information about their prior ASL Studies coursework experience. From those who applied, I used purposive sampling to select three students who were part of this study from winter 2019-spring 2019. All three students signed an informed consent form to indicate their agreement to participate in this study (see Appendix B). 
At the beginning of the winter term, I set up individual appointments with each student to explain the purpose of my study. I provided each student with a copy of the upcoming Deaf events and encouraged them to participate, as they were able. These events were on campus and open to the public, so students were not required to attend to participate in this study.

During winter term, I scheduled interview sessions (see Appendix C) with each participant to learn more about their history, their experience with ASL prior to arriving at WOU, their experience with the Deaf community, and their interest in becoming an integral part of a Deaf CoP. I asked students about their perceptions related to the ease of accessing the Deaf community and the ways their experience might change (positively or negatively) if they had more opportunities to interact with native members of the Deaf/ASL community in Oregon. I also asked about whether or not they felt their experience helped them transition past the LPP they had experienced prior to involvement in the community. I also included questions related to how students plan to use ASL in the future, specifically in a graduate program or in employment.

Interview protocol. I developed an individual interview using a combination of structured and semi-structured items for this research study. For each interview, the structured portion focused on obtaining demographic information and identification of prior history and exposure to ASL. The semi-structured portion of the interviews helped address the three research questions. The interview protocol included 16 questions (see Appendix C) with the option to add or delete questions as necessary. My colleagues at WOU (full-time ASL Studies instructors) were vital in the process of reviewing my 
interview questions. They offered valuable feedback and provided input regarding how the interview questions addressed the research questions. Importantly, I included my colleagues in each step of my research, because I believe transparency is critical.

I conducted the interviews individually at WOU, and I used audio and video recording during these interviews to capture both English and ASL responses. I captured the spoken English responses of each student via audio recording, and sent those files for transcription. I also video recorded the interpreter, so I could review participants' comments in ASL as necessary.

Role of the researcher. As a second-generation Deaf woman, whose first language is ASL, I recognize that I brought a unique lens to the research that non-native ASL users could not bring. I also brought 15 years of experience teaching ASL as a second language to hearing students. Based on my experience, I believe interaction with native ASL users is one of the most effective ways students can improve their ASL skills and fluency, but I recognize that it is difficult for students to find the time to immerse themselves in Deaf/ASL CoPs.

As an ASL instructor, I have engaged with students at various points on their language acquisition journey. I have experienced teaching all levels of ASL ( 9 courses over the span of three years) and many elective courses related to ASL (e.g., Deaf Culture, Deaf History, Mental Health in the Deaf Community). This experience had afforded me the opportunity to notice patterns in language acquisition that tend to surface for students who were trying to learn ASL as a modern language. These patterns were what led to my initial interest in this topic for my research. 


\section{Data Collection and Analysis}

I used a qualitative descriptive case study design for my research. Yin (2002) defined a case as "a contemporary phenomenon within its real life context, especially when the boundaries between a phenomenon and context are not clear and the researcher has little control over the phenomenon and context” (p. 13). According to Yin (2018), not until recently were case studies accepted as a method of research instead of an exploratory measure embedded in another type of research method. He clarified, ... case study research comprises an all-encompassing mode of inquiry, with its own logic of design, data collection techniques, and specific approaches to data analysis ... case studies are not limited to being a data collection tactic alone or even a design feature alone. (p. 16).

A case study approach was an effective and appropriate design for addressing my research questions because it allowed me to (a) evaluate students in context, (b) have ongoing access to these students during the 2019 academic year, and (c) explore what students were thinking and feeling relative to their experience in the Deaf community. Based on my analysis of the interview data, I identified common themes students experienced related to barriers and opportunities to interact with native ASL CoPs. Participants shared additional data about competing priorities during interviews that helped me understand the underlying reasons students might not be able to make the time to attend events, even if they knew such interaction with language models helped in their language acquisition.

In this study, I used purposive sampling to select three students for participation.

First, students enrolled in ASL 8 during the winter 2019 ( $N=65$ students) received an introductory email inviting them to apply to participate in this research study. To 
participate, students had to be taking ASL courses during the 2019 academic year and willing to participate in my dissertation research; this limited the pool of participants. From the group of students who expressed an interest in participating, only three met all of the criteria to participate in my case studies. To view my problem of practice through as many lenses as possible, I selected one student with no prior exposure to ASL before entering WOU, one student who took ASL during high school, and one transfer student with prior experience in ASL for another community college/college/university. I chose these categories because they were representative of the three different types of students that commonly enroll in our ASL Studies program. During the past 10 years, the number of transfer students who take ASL at WOU gradually increased, and I wanted to understand how their prior experiences in the Deaf community might influence students' perceptions and experiences apprenticing into a Deaf CoP. I used purposive sampling (Seidman, 2013; Yin, 2018) rather than random sampling to ensure I recruited students to participate who were willing and able to participate in the interview process; ones who and could provide honest reflections of their experience as part of Deaf CoPs.

I conducted one interview with each participant in a small classroom. These interviews lasted approximately one hour, and I did both video and audio recording on them for data analysis purposes. I uploaded these videos to a software program called Go React that allowed me to review the videos and make English notes or record ASL notes at specific moments during the interview for coding purposes.

Data analysis. I used an inductive analysis approach to review the interview data (Merriam \& Tisdell, 2016). According to Merriam and Tisdell (2016), the objective of an 
inductive approach to data analysis in multi-case studies is to find patterns or similarities (units) across cases that can lead to answering the research questions posed in my study. Because I had three students, I needed to conduct within case analysis and cross-case analysis (Merriam \& Tisdell, 2016). First, I examined each case (student) to find relevant themes, and then I compared those themes across cases to identify where similarities across all cases exist. I used these themes in my final findings report.

Validity and reliability. Validity and reliability in qualitative research are more difficult to ascertain than in quantitative research (Maxwell, 2013). Originally, I planned to conduct all interviews in ASL, but I realized that this would limit the students' abilities to share as much information as possible, due to them not being fluent in ASL. Instead, I hired an ASL interpreter to facilitate communication during all interviews. Students had a copy of my interview questions, and when I asked follow up questions, the interpreter voiced those questions to the students. Students used spoken English for their responses, and the interpreter communicated their answers to me into ASL. After each interview, I sent the audio recording to Rev (an online transcription service) to be transcribed. I engaged in member checks with students to ensure the accuracy of their transcribed comments. According to Merriam and Tisdell (2016):

The purpose of member checks is to take your preliminary analysis back to some of the participants and ask whether your interpretation "rings true." Although you may have used different words (it is your interpretation, after all, but derived directly from their experience), participants should be able to recognize their experience in your interpretation or suggest some fine-tuning to better capture their perspectives. (p. 246)

I also engaged in data triangulation by comparing my findings across the three cases, and with comparable studies in the literature and asking for feedback from other 
faculty in the ASL Studies department during this process. According to Merriam and Tisdell (2016), “Triangulation using multiple sources of data means comparing and crosschecking data collected through observations at different times or in different places ... or from follow-up interviews with the same people" (p. 245).

To increase the credibility or validity my results, I engaged in data triangulation by comparing the three sets of interview data. I also had other ASL faculty members review the data sets and my analysis. According to Merriam and Tisdell (2016), "Triangulation using multiple sources of data means comparing and cross-checking data collected through observations at different times or in different places . . or from followup interviews with the same people" (p. 245). My goal was to ensure the trustworthiness of the data and the credibility of my results. 


\section{Chapter 4: Results/Analysis}

In March 2019, while writing this dissertation, The Chronicle of Higher

Education listed WOU as the second leading university to confer ASL Studies degrees in the United States ("Which Colleges Grant," 2019). Because WOU is in a small rural town, it has limited access to a large or nearby Deaf community, so students have few opportunities to engage with native users of ASL. The purpose of this study was to explore whether attitudinal and situational barriers make it difficult for students to move past LPP (Lave \& Wenger, 1991) in the Deaf community and consider possible ways to support students on their language acquisition journey.

While a plethora of general research exists on second language acquisition, as presented in Chapter 2 of this dissertation, little research focuses on students' perceptions on the importance of engaging with native language models when acquiring ASL. The purpose of this study was to add to the body of research in this field and to identify some of the opportunities and barriers that students perceive influence their experience apprenticing into the Deaf community. My research study followed a qualitative case study design and my analysis included both comparative and descriptive lenses for understanding the stories of three participants shared during the interview process. All three participants were students studying ASL at WOU.

\section{Analysis of Data}

In my original research design, I planned to interview each participant using ASL. I also planned to have an ASL interpreter watch the video interview of each participant, 
and provide a spoken English voice translation for each. I intended to send those voice translations to a transcription service to make English transcripts for me to review. The participants all agreed to conduct the interviews in ASL; however, a few days before the first interview, I decided it would be more effective to collect participant perceptions and stories in their first language, instead of trying to use a second language (ASL). I reached out to all three participants, and they were amenable to changing the language of the interview to spoken English. This change did seem to create a slight bit of incongruence for each of us because as part of Deaf culture, students learn to use ASL in the presence of a Deaf person, not to revert to spoken English, which is inaccessible. After the initial newness of this experience wore off, the participants were able to move forward with the interviews in spoken English without any further issue. It was a moving experience for me to see each of these participants initially hesitate when asked to change from using ASL to spoken English. Further validation for me was their desire to be part of the Deaf community and their sincere interest to be involved in my research study to inform our practice.

I chose to include three participants in my study because each represented a cross section of students who attend WOU. The three most common types of students who enter the ASL Studies program at WOU are:

- Students who took ASL classes starting in high school and continued taking classes at the university

- Students who took ASL classes starting in community college and transferred to the university for additional classes

- Students who had no prior exposure to ASL classes before arriving to the university 
Overall, students across the three cross sections shared many similarities; however, I also noted key differences as I learned more from each participant.

In this chapter, I introduce each participant and highlight key points from their interview narratives. Then, I share my findings relative to my research questions, and finally, I address the limitations of my study. The three research questions that guided my study were:

1. How do students in an ASL program at a rural west coast university describe opportunities to engaging with native ASL CoPs?

2. In what ways do students learning ASL at a rural west coast university feel a part of the Deaf/native ASL CoP?

3. What experiences helped students apprentice into the Deaf community? With these research questions in mind, I journeyed into the lives of three ASL studentsparticipants in my study — who were brave enough to share their story. The first interview was with Cameron.

Cameron. Cameron is a 19-year-old, white female, who is currently in her junior year at WOU. She appeared to be energetic, talkative and had an outgoing personality. During the interview, she was open to answering questions, happy to provide detailed information, and told of her experiences from a positive lens. Growing up, Cameron had some exposure to ASL and knew about the Deaf community through her great aunt who was an ASL interpreter. Cameron's formal ASL instruction began when she took ASL classes during her junior and senior years of high school. Her high school was located near a midsize Deaf community on the outskirts of Seattle, Washington. After graduating from high school, she attended community college where she took an additional two years of ASL course work before transferring to WOU. At WOU, Cameron has been 
taking ASL 9, the last core ASL course offered in the ASL Studies program. She disclosed that she lives in a dorm on campus that has an ASL hall where students are in ASL courses and use ASL to communicate with one another as a means of practice and immersion.

During high school, Cameron attended as many Deaf events as she could, but these events were often located far distances from her home, leaving her to rely on her parents for transportation to these events or for permission to attend. She revealed that her parents were supportive of her desire to learn ASL. Cameron shared:

A lot of the Deaf events were an hour away, so my commute as a high school student was limited with my parents' permission for me to drive that far, who's going with me, how am I getting that access. That was more of an issue, but I definitely made it work because my parents knew how invested I was. The more effort that I put in, the more opportunities they'd allow me to have because it definitely started as an interest. I took Spanish and that was a disaster, and so I tried ASL and knew that it was an interest from actually attending an event where there were Deaf individuals. I didn't quite realize it was a deaf event or a deaffriendly event at the time.

During these events, Cameron often struggled with the decision of whether to disclose that she was an ASL student because she felt like the Deaf community members would view her attendance as a course requirement, rather than her genuine interest in participating in the activity. In general, when she attended events during high school, she felt that Deaf people viewed her as only attending because she had to fulfill a course requirement.

Cameron recalled the first time she knew she wanted to be an interpreter was after meeting a Deaf man who was homeless at a holiday event when she was young. Nobody at the event knew ASL, and Cameron stated that it was a defining moment for her 
because in that moment she realized she could bridge a communication gap by knowing

ASL. She recounted:

My first experience, I feel like everybody who wants to become an interpreter has their first experience, but mine was, I was working at a homeless shelter with my mom around the holidays and we were taking Santa pictures and I had a Deaf man come up to me and wanted his picture with Santa. And, probably in his 60s, so he is very excited about this. Not an opportunity that happens frequently and he immediately pulled out a pen and pencil knowing that I'm a hearing person and I can't sign. And realizing how stuck he felt and realizing that, "Okay, I have to do this because other people don't know." It was very frustrating for me as a hearing person because I realized that I could be learning a language, I could be doing other things, and made me want to have that communication. And, so I got involved because of that.

I thought Cameron's story would end with her sharing about her experience meeting that

Deaf man at the shelter, but I was wrong. She went on to explain that her true reason for learning ASL had more to do with her own story and the experience of her siblings than the experience of the man she met at the shelter that day. She explained:

I really want[ed] to get involved because I have younger siblings who are both in elementary school and the further that I got into Deaf culture and things that happen in the Deaf community now, I realize that Deaf education in mainstream schools is not ideal, and that a lot of interpreters are not certified or qualified enough to be interpreting in a school district. And I took it to a personal level to compare with my siblings, because if my little sister were to come to me and tell me that her interpreter at school is not allowing her to ask questions or isn't giving her that opportunity to learn when I know that that's what she wants, it was heartbreaking.

This lens was particularly interesting because rather than imaging what life might be like if she was Deaf, Cameron started to imagine what the experience of her younger siblings would be like if they were Deaf. Young children are often inquisitive and love to learn, but without access to fluent language models or people who understand their language (e.g., interpreters, teachers who know ASL), students are limited in their capacity to participate in school, sports, clubs, or with their peers. Because Cameron had experience 
watching her siblings learn and grow, she saw the power of language, which may have informed her thoughts about her future career and how ASL as a language fit into those plans. She commented:

I want to be able to be an educational interpreter who's qualified, who can work to not take over, because I think that it is Deaf people who run and who can fight their own battles, but to assist in that. I want to be someone who can offer these kids a brighter education to have them have full and equal access. I don't need to help. I think the Deaf people can do it on their own, but I think that if I could be involved in any way that it just makes me feel like I can make a little bit of a difference as a hearing person. It's not necessarily a negative label that I'm a hearing person who is clueless, but I'm taking the time, I'm learning these things about the culture, I'm learning these things about the people, and how can I, as a hearing person support [them]?

Cameron's explanation about supporting the Deaf community and not "helping" seems indicative of her exposure Deaf mentors who have exposed her to this frame. Even with all of this exposure to ASL and the Deaf community—beginning in high school, continuing on in community college, and at the university, with hopes of getting accepted into the interpreting program - Cameron still questions her place in the Deaf community. When asked about where she fits within the larger Deaf CoP, she indicated that she still sits on the outside of the most central circle within the Deaf CoP. When I asked her to talk about her own experience of trying to apprentice into the Deaf community, she explained:

I went to a Deaf art gallery where all of the artists were Deaf. And one artist made a periodic table where different elements were different colors and mentioned that the interpreters aren't in the Deaf community, and that was a perspective shift for me. I had assumed that interpreters were in the Deaf community, and they're not. They're supporters and they're there, but they're not Deaf themselves. They don't have the Deaf culture and so it's not their community to be in.

Apprenticing into a community often requires a steep learning curve. Trying to figure out how one fits into this new community can be a delicate process. Cameron's experience at 
the art gallery made her realize that just because she intends to work in a Deaf related field (as an interpreter), does not automatically mean she is part of the most intimate circle of the Deaf community. If Cameron had this same experience and left feeling insulted or devalued after learning she would not be part of the most intimate circle, she might have changed her mind about her future career. Having an open mind, and understanding that within marginalized communities there are often barriers that cannot be crossed by members of the majority community, allowed Cameron to respect the artist's comments and still value the ways she could fit into the Deaf community. I asked her how she felt about being on the outside of the most intimate level of the Deaf community, and she responded:

Okay, so I don't think that I will ever make it into the core level. I think that the Deaf community isn't meant for hearing people. I think that it's a fine line and can I definitely sit on the board and be supportive? Of course, but it's not my community to take over, or my community to fill. But, I think that I am on the line, but I think that I'll sit on the outside, which is a perfect and an okay place for me to be as a hearing interpreter.

As a follow up question, I asked her she pictured fitting into the core level of the Deaf community, and she explained:

Someone who is capital "D" Deaf and has Deaf-Heart is who is in the community. I learned about Deaf-Heart in ASL 6. That it's not a mental thing, it's a heart thing and it's how you yourself are identified and how that person decides to be active in that community and continue the language and . . . culture and saying, "I'm involved with you and you're involved with me, and we're in it together."

I pressed a little more, and asked her to try to differentiate between those on the outside and those on the inside of this most intimate core level. She took a minute to contemplate and added:

I think that a Deaf person who identifies as Deaf, who is proud of being Deaf, who is just really there to be supportive of other Deaf people, to realize that it's 
not a disability that, sure for paper I have to put it down if I want an interpreter, but I have my own group of people. I'm still learning. I think that maybe my opinion could totally change, and maybe I'm biased or maybe I'm skewed from other teachers because I've realized here at Western that some of things I learned before maybe aren't true or maybe don't fit.

It was interesting to see her change in stance as the interview progressed because it was clear that Cameron was trying to sort through all she had learned over the years regarding who is part of the Deaf community. At the same time, she remained open to the possibility that her ideas and thoughts might need to shift to align more closely with the Deaf community here in Oregon, specifically at WOU. One factor that that seems to have contributed to this is the fact that Cameron has had exposure to Deaf instructors who have hearing spouses. She shared:

I was taught that hearing people tend to sit on the edge, that you could be close but maybe not in, but maybe I'm wrong and maybe there's some hearing people that can go in. Because listening to some of my teachers talk about their spouses and how they might be included even if they're a hearing person, if they're involved and they're living a Deaf culture filled life.

This flexibility in her thinking is a strong indicator that she will be able to learn and grow as she continues to interact with more members of the Deaf community.

One important factor for language acquisition is access to fluent language models (Krashen, 1988; Vygotsky, 1978). Cameron shared that coming to WOU brought her more opportunity to engage with native language models on campus because all of her ASL instructors are Deaf and that she felt that was a huge benefit because she was immersed in Deaf culture and the Deaf community every day. She noted that this experience made it easier for her because she did not have to fit going to events into her schedule. She mentioned that when she first arrived to WOU, however, she felt that her skills regressed because her ASL classes did not require her to attend Deaf events. This 
meant that she no longer had to push herself to go out into the community and meet members of the Deaf community who might sign differently than those she was used to interacting with at WOU.

When asked about anything else that I should know as the researcher of this project, Cameron offered two key sentiments for my consideration. First, she disclosed that she had been accepted to Gallaudet University, which is the only liberal arts university for Deaf people in the world. In general, most of the students who attend Gallaudet are Deaf, and so being accepted at Gallaudet if you are a hearing student is a big honor. I was surprised to learn that she turned down this opportunity, and when I asked for her rationale, she explained:

I applied to Gallaudet University and got accepted and I chose not to go. I went, it was a beautiful campus. I have a Deaf friend who goes and absolutely loves it, and I knew the opportunity that Gallaudet would allow me to have, that their program is phenomenal and it is only Deaf college in the country, so if I really was to go to a Harvard of interpreting programs, that would be my choice.

Cameron expressed her goal of wanting to go into the field of interpreting, so her decision not to attend the interpreting program at Gallaudet was not one she took lightly. She went on to explain that she chose WOU because in addition to the interpreting program, there were many other positive factors that drew her to this university. She explained:

I think that as a student, a big part of my decision about coming to WOU was the community base. I really fell in love with WOU and the opportunity that it gave me. And, I know that my skills could take me to Gallaudet but I knew that here at WOU I was getting a lot of other experiences. I also I am a small-town person so D.C. was a little overwhelming. But I chose to come to WOU and its benefited me in every way possible. Even though coming to WOU was a risk because I had an extra year of prereqs here, and I am applying for the interpreting program this year, versus at Gallaudet I'd go right in. And so, I personally have to apply for the program, but I feel as though it's paying off. But from someone who had the 
opportunity to go to the top as a hearing person in that I read a lot, and it's a $10 \%$ hearing community for the college, I think that it would been a great experience, but very happy with the opportunities that WOU has given me.

Cameron had applied for the interpreting program at WOU right before our interview.

She was not sure if she would be accepted into the program, which made me wonder if her experience at WOU had been worth the risk, even if she was not accepted. In response, she said:

I have definitely had conversations with my parents. They asked me for about a month if I was sure about my decision for WOU and I told them that I was, and they checked in with me this weekend at family weekend and asked me if I'm still sure about my decision. And, they told me that I was glowing as I was describing to them my experiences and that I absolutely enjoy my teachers and that I feel so blessed to have the opportunity [to be here].

I am excited to keep an eye on Cameron to see if she gets accepted into the interpreting program and how that continues to shape her language and LPP into the Deaf community.

Alex. Alex is a 26-year-old, White/Mexican American female, who is currently in her junior year at WOU. During the interview, she appeared to be confident and comfortable sharing her experiences, and seemed to enjoy our interaction. Alex presented herself as a down to earth, calm, and reflective person as well as one with critical thinking skills and a social justice lens. Alex's initial exposure to ASL began during community college. Located near CSUN, the community college she attended was surrounded by a vital Deaf community. CSUN is home to a thriving Deaf Studies program and boasts more than 200 Deaf and Hard of Hearing students. Many of the faculty who teach in the Deaf Studies program, and most of those who work in the National Center on Deafness at CSUN, are Deaf. Alex stated she believed that having so 
much exposure to a diverse group of Deaf and Hard of Hearing people had a huge impact on her language acquisition and her desire to apprentice into the Deaf community.

Alex took her first ASL courses in 2012 during community college. Then she took a three-year break and did not take another ASL course until coming to WOU in 2016. Her first ASL course at WOU was ASL 5 given her transfer to WOU during winter term. ASL 5 is the second core language class in the second year of the ASL Studies program. At the time of our interview, Alex was completing ASL 8, was registered for ASL 9, and was a pre-interpreting major. Alex recently contacted me to let me know that she was accepted into the interpreting program at WOU and will begin in the fall 2019.

Unlike Cameron who had limited opportunity to interact with the Deaf community when she first started taking ASL classes, Alex got involved immediately in local Deaf events when she started learning ASL. A close friend of hers, who originally encouraged her to take ASL courses, often went with her to various events. In the beginning, attending Deaf events was not something Cameron chose to do voluntarily. She was required to attend three Deaf events per semester, so her involvement in the Deaf community started because her grade was contingent upon her attendance.

I asked Alex to tell me a little about her experience taking ASL during community colleges. She shared:

My experience was, when I first took up ASL, I had a hearing teacher. She was actually amazing. She really encouraged us to learn ASL, and to be a part of the Deaf community, and I loved it. She actually is what got me into ASL. And then the next time I had a Deaf teacher, she was also fantastic. They really, really pushed going to Deaf events. More so than I think here. But there, I feel like the Deaf community, there's more Deaf events in general, so I felt like I had more of an exposure to the community, as opposed to when coming here. 
Importantly, over the years, the ASL Studies department at WOU has gone back and forth on the number of Deaf events that are required for students to attend each term. Originally, students were required to attend three to five events, but because there is such a small Deaf community in our rural area, it was difficult for students to find events to attend. Additionally, when the Deaf community hosted events meant as Deaf socials, students would often attend and this meant the loss of meaningful Deaf space. For the past several years, students are only required to attend two Deaf events each term.

When I asked Alex about the ways she gets involved in the Deaf community here, she listed several experiences: coffee chats, Deaf Night Out (DNO), and ASL Club. On most Fridays, a coffee shop in Salem holds a Deaf coffee chat, about 20 minutes from WOU's campus. Second language learners often fill this event, and Deaf people do not always attend. This is a popular option for some students because Friday night is at the end of their school/work week, which makes it easier for them to attend this event. Because there are not always native language users at this event, the opportunities for language acquisition or apprenticeship are limited. In contrast, DNO, is a monthly event hosted by Deaf professionals in attempt to bring together the Deaf community for a chance to unwind and connect. A long-standing DNO takes place in Portland, but summer 2018 brought a local DNO to Salem. The first event was mostly Deaf people, yet once students learned about this event, many started coming and the opportunity for Deaf space decreased. Deaf programs face a fine balance between making sure students have opportunities to engage with native language users, and hold sacred space for the Deaf community to come together without the presence of others from the majority culture. 
Alex reported her active role in the ASL Club is a WOU sponsored club that takes place each week on campus. The club leadership appointed Alex as the secretary for the 2018-2019 academic year. This means that she has been s involved in most of the activities that ASL Club sponsors, and most of the ASL Club events. She was instrumental in hosting a campus-wide event to raise awareness about the Deaf community called ASL City. According to Alex:

ASL city is a day in a Deaf life. So, you have tables out where you could set up to get coffee, but you have to use sign language for it. It's really just exposure to the Deaf culture. You know, we have different tables for Deaf history, the old technology that Deaf people would have to use to communicate with each other. This year we're going to have a DeafBlind table. And so, just basically things that will expose other people who may not know very much about the Deaf community.

ASL City is not new to WOU, in fact, the original idea started in 2011 with a student I was working with for her practicum. The event ran every year until a few years ago. Eventually ASL Club decided to host this event, and it is often a favorite for students, faculty/staff, and the local community. The crowd it draws to WOU has varied from year to year, sometimes consisting of high school students learning ASL, local community college and college students, and sometimes Deaf students from the Oregon School for the Deaf (OSD). This event was meant to expose people to Deaf Culture and bridge the gap between the hearing and Deaf communities, but there are often more hearing people who attend than Deaf people. While this event has provided great exposure and an opportunity for people to learn about ASL and the Deaf community, in terms of developing language fluency and second language acquisition, students must engage with native language users for this process to be effective (Gilardi \& Guglielmetti, 2011; Krashen, 1988; Vygotsky, 1978). 
Based on Alex's explanation of her limited opportunities to engage with Deaf community members in the WOU rural area, I asked her follow up questions about her experience with the Deaf community in Los Angeles, particularly the community that was close to her community college. She noted that one of the most significant differences, aside from the number of people, was the demographic of people who tended to show up to events. In Los Angeles, she shared that events tended to bring in a younger crowd. According to Alex:

I would say the Deaf community, from where I'm at, is a lot larger, and has a lot more younger Deaf people who would go. So that made it a little bit easier, wasn't just older adults who you had to talk to, and didn't make that kind of real connection with. But, with younger students, or younger people there, it was easier. Like, "Oh, well let's go hang out and do something, because we're the same age." So it's a little bit more of a connectedness there. They didn't necessarily have, from my knowledge, a DNO specifically. It was always like, "Oh meet us at a pizza place. Let's go to a Starbucks. Let's go somewhere, all of us, and just meet and chat." And, that's where I felt like it was a little bit more inclusive.

The idea of really connecting with a community, or someone from the community, gets to the heart of the idea of apprenticeship and moving toward LPP (e.g., authentic engagement with the Deaf community). Instead of showing up to events to get credit for an assignment, the idea of interacting with fluent language models who actually share something tends to lead to more natural conversation and connection. Finding an old timer to help guide the apprentice experience is essential. Alex told me a little about a friend who filled this role for her, saying:

I would say the most [connected I felt] would be in the Deaf community when I was back in California. I also worked with, closely, a hard of hearing individual who, him, and I would sign all the time while we were at work. But, since I've gotten here, the most interaction I have with ASL users, Deaf or hearing, is here at school. 
Alex did not explain why there was a difference between interacting with someone at school versus at work, however, her tone seemed a bit remorseful at the missed opportunity to have someone to engage with in that meaningful way. When I asked her if she was willing to attend Deaf events on her own, she noted that while she would be willing, she would much rather attend with someone she knows. She shared, "I think I would go with at least one friend, but if I go by myself, I'd go by myself, and hope that I'd be able to build enough confidence to start conversing with another person, instead of just watching everybody." I was interested in learning about the types of people Alex felt like she would connect with at Deaf events, and I was not surprised to learn that she was more interested in finding a younger crowd of people who shared similar interests and experiences. Throughout the interview, a common theme that came up in Alex's responses related to her desire to build connections and relationships organically, not forced because of a class requirement. In other words, Alex wanted authentic experiences with members of the Deaf community. She made it very clear that the idea of striking up a conversation with a Deaf person with the purpose of "practicing" ASL was not her interest. She wanted to find ways to interact with Deaf community members in ways that led to lasting connection and relationships. She shared:

I guess I don't want to feel like it's my place to just assume that Deaf people need to talk to me because I'm a student who wants to learn. I definitely feel like it is my place to go up and meet somebody, but at the same time I don't want it to feel fake like, "Oh, this is just another student coming in and meeting Deaf people just to say that they've met a Deaf person and they practiced signing." You know I would want it to be a little bit more organic than that. So that's why having younger people in Deaf events I feel more comfortable, because I can have relatable topics to talk about and stuff. 
That desire for relatable topics is an important point to consider, because when trying to learn a new language, it is important to interact with people who are talking about content that is of interest, because there will be more motivation to remember what is learned. Alex reiterated that she did not want to impose on Deaf community members just for getting to practice her language skills. She said, "I'd rather it just kind of be like, I'm introduced to that person, we have a few shared interests, and then continue the relationship on from there."

For Alex, the issue of having authentic experiences with the Deaf community related to the small size of the community in rural Oregon, especially one consisting of older members, and to the limited opportunities to engage with peers with similar interests. She also shared that she would rather connect with a group of Deaf people who were just going to hang out somewhere, and not necessarily attend a planned or purposefully hosted event with the intention of bringing people together.

As we were winding up our interview, I asked Alex where she felt she fit in the Deaf community relative to the model (see Figure 1) that I shared with her on the board. After careful consideration, she admitted that she currently is in the outer circle, which indicates someone taking ASL for credit. She lamented about this being the case and expressed that she would rather be closer to the heart of the Deaf community. She explained her goal for her time at WOU:

I would say, after completing here at Western, I would like to have basically the confidence through my ASL learning through all the classes, with the language, learning more about the culture, the community. But at the same time having that confidence to go to the Deaf community. Creating those relationships with those people, I would hope at the end of it I would be in the last [most inner] circle. You 
know inside the Deaf community, have friends and people that I just go and just be a part of the Deaf community with. That's what I would hope for my

When I asked her what it would take to be able to move from Level One, taking ASL for credit, to Level Three, the core or most intimate level of the Deaf community, she seemed confident she could make that transition easily. Her response to my inquiry was:

I honestly don't think it would take much time, it just is me pushing myself to go out to those Deaf events. I was thinking about maybe going up to Portland for a Deaf event. Because maybe they would have more of a crowd that I would be more relatable to, younger even. And it also seems like they might have a little bit more different variety of Deaf events. Not so much just meeting up at a restaurant and hanging out. So I don't think it would take much time at all, it's just me pushing myself to go out to Portland and be a part of that community.

The two themes that stood out in my interview with Alex were (a) the desire for real connection in the Deaf community, and (b) the challenge of finding that person in Oregon given competing priorities (e.g., Portland is far, the local crowd is older, lack of common interests, and so on). What became clear was that while Alex has the desire to want to connect authentically with the Deaf community more, she identified barriers that seemed to make it difficult for her to make this a reality. I found these same barriers echoed throughout the interviews with both other participants.

Blair. Blair is a 19-year-old, Asian, Pacific Islander (Hawaiian), and White female in her junior year at WOU. Her major is business with a focus in accounting and finance with a minor in ASL Studies. She felt a little nervous during the beginning of the interview, but as we talked, she warmed up and was able to share a lot about her experience journeying into the Deaf community. Blair was born and raised in Maui. During elementary school years, Blair started to learn to read and write Japanese and grew up speaking Japanese with her grandfather. In middle and high school, Blair 
attended a private Hawaiian school and had to learn Hawaiian language and culture. Later, in high school, Blair took additional Japanese language courses, but she does not consider herself fluent in Japanese or Hawaiian language. In total, she has had exposure to four languages (the other two being English and ASL). What became clear right away is that Blair had an appreciation for language, culture, and connection with others.

What makes Blair unique is that she had no exposure to the Deaf community before coming to WOU. Growing up, her mother was the director of the special needs program in the local elementary schools, and Blair often volunteered in the schools and worked with students who were non-verbal. She learned some basic signs in order to communicate with the students, but never learned ASL or interacted with anyone who was Deaf, until taking courses at WOU. Having come to WOU without prior exposure to ASL or the Deaf community, Blair was unable to speak to whether her experience with the local Deaf community in rural Oregon was similar to what others experience in areas with larger Deaf populations. Compared to the other two participants, the focus of my interview with Blair was solely about her LPP into the Deaf community based on her interaction with local Deaf community members.

Due to a more limited scope of experience in other Deaf communities, I decided to frame the remainder of the questions in the interview around the concept of being part of a Deaf CoP at WOU. I was curious if Blair had any prior knowledge of what the term CoP meant, and to my surprise, she was able to articulate a definition of a CoP, and explain her role in the local Deaf CoP. She shared:

[A CoP is] the concept of learning with a group of people who are interested in the same thing but I don't have like specific research or any knowledge of it. I'd 
say most of the friends that I've made here at WOU have been within the ASL community or within my ASL classes and the program. So, like I've built friendships through that and I feel like we're a lot closer because, especially at the 300 level of ASL classes, people are in them for a career and they're in them not just because it's for their degree credit, so they're a lot more passionate about it and a lot more willing to help each other and support each other in learning.

Blair seemed to reframe her idea of a CoP based on experience with other hearing second language learners. She followed up her definition with a description of her experience as part of CoP of students learning ASL, noting how difficult it was to attend Deaf events alone when first getting started and while still not confident in her language skills. I thought it was fascinating that Blair automatically reframed the idea of a CoP to be about her experience learning with other hearing second language learners; as opposed to thinking about her journey into a Deaf CoP. I think this is particularly interesting because she followed up her definition of her experience as part of CoP of students learning ASL, by sharing how difficult it is to attend Deaf events alone when first getting started and while still not confident in her language skills. This is a lament that students often share when asked about their reservations about attending Deaf events. I found these same sentiments echoed across all three participants during our interviews together.

The idea of a community of hearing students all working together to support one another in their language acquisition is a beautiful one, yet it is different from a CoP in which a newcomer apprentices into the Deaf community by way of an old timer. This idea of students banding together and supporting one another was not surprising, but it left me with more questions. I was curious about Blair's apprehension around attending events on her own, especially since she moved from Maui on her own and had to develop a new community for herself here at WOU. She shared: 
I think before one of the main barriers, even if I did have the transportation, it was feeling comfortable especially if you didn't have friends and you weren't able to communicate as well. That's something that's very nerve-wracking to go into a situation where you know peoples' experience and their abilities are a lot higher. Now, I'd say it's still the same like going to events, it's definitely a lot more comfortable with friends than going by yourself but now I feel like that barrier is not there as much.

This led me to question whether Blair had ever had negative interactions with members of the Deaf community, particularly during a Deaf event that might have caused her apprehension. I was a little nervous to ask this question because the community around WOU is so small. Yet, at the same time, I know our community to be inviting and supportive, so I did not $t$ anticipate that Blair would have had a bad experience. She explained:

Pretty much everyone I've met within the deaf community have been extremely welcoming, like extremely patient especially when I couldn't sign at a conversational level and I was still very awkward with signing, they were patient and would like explain signs that I didn't know or would fingerspell things multiple, multiple times. And that made it a lot more encouraging to go. I think if my first experience going to a deaf event, especially at a low level, was bad, or if I was met with like just a snobbiness or a strictness, I probably wouldn't be interested like I am now.

Blair shared that while she had not personally experienced any negative interactions with people in the Deaf community, some of her friends had shared negative experiences with her. She mentioned that the negative comments she had heard had come from students taking ASL at other schools, and not from anyone taking ASL from WOU or interacting with the local Deaf community. While she did not elaborate, she did say that there were complaints from students who were trying to learn ASL online and then going out to engage in the community. 
Blair's motivation to continue to learn the language is of particular interest to me because she does not plan to go into a field that requires her to use ASL to communicate. Her career goal ties to her dedication to attending events, challenging herself to become more fluent, and her commitment to engaging with members of the Deaf community. Her intention also seems to come from a sense of wanting to be part of the community, and not necessarily from a frame of how she might be able to benefit long-term by engaging with Deaf people. Blair disclosed her interest in being part of the Deaf community; she told me:

I think it just comes from the fact that I, like I grew up with no experience with the Deaf community, never really seen ASL as a language and to come here to a place where it's so prevalent and it's such a big thing here it's been pretty eye opening and it's been a language and like a culture that I've fallen in love with and it's something that I wish a lot more people had the experience.

This same idea was brought up in my conversation with Alex, because even though she grew up in an area that has a large population of Deaf people (Los Angeles), she never met a Deaf person or interacted with the Deaf community until taking an ASL class during community college. She shared that it was like finding out that there was this huge community of people who had their own language and culture living in her backyard, but that she had lived for so long without ever realizing it. Once a person becomes familiar with the Deaf community, they often can take two paths. The first is to get involved on the periphery and learn ASL as a second language for credit, and the second is to get involved in the Deaf community because of a genuine desire to share, work, play, and experience life with Deaf people. Those who are interested only in taking ASL to fulfill their modern/foreign language requirement do not often stay involved in the Deaf community once they complete their coursework. This is significant because it means 
that Deaf community members are investing in people (e.g., mentoring or giving feedback to ASL students who attend community events) who are only going to be involved in the Deaf community temporarily. This can lead to Deaf community members feeling used or feeling as if they are being seen as a token.

Blair shared that she had originally attended Deaf events because they were required for her ASL courses; however, she was only required to attend one event each term, she still found herself attending three or four events, simply because she loves the language and the culture. The types of events she attended each term were similar to those of the other two participants. Over the past several years, Blair had attended events ranging from ASL coffee chats, events hosted at the OSD, interpreted plays (locally and in Portland), ASL Club, and the community events now hosted at WOU. She shared that it is now much easier to attend events because she has a car and a community of friends who tend to go to events together. When I asked what type of event she felt she benefited from the most, she shared that they really enjoyed the community events hosted on WOU's campus that we started winter 2019 term. She said she felt like these events brought a large number of Deaf community members together, ones who were interested in connecting with WOU students, and how this felt different from many of the other events in which she had participated.

Knowing that Blair does not plan to work in a Deaf related field, I was curious where she felt she currently was in terms of connection in the Deaf community as indicated by my model. She stated that she felt like she was in the second ring, which meant that she was not just taking ASL for credit, but that she was also not in the core 
layer/intimate layer of the Deaf community. She explained that she felt like with all of her interaction and engagement with friends and at events, that she was journeying more toward the center of the community, but she recognized that if she did not keep taking courses that required her to stay current with the language; she might not stay at that level in the community. She disclosed:

If it's something that I don't keep up with linguistic-wise, I don't think I'd be able to move towards number three [the core level of the Deaf community]. I think I'd stay towards two. I've done a few internships and stuff like that for business specifically and I've gotten to see how they use language or working with people who don't speak English.

Notably, during the interview, Blair was able to reframe our discussion in terms of her own experience and consider how my questions related to her personal experience. While I did not ask her about her interaction with others who work with consumers who are not native English speakers, she was quickly able to use this frame a reference for why she knows she wants to integrate ASL into her practice so Deaf people feel more accommodated. She shared:

When I was doing my past internship at a brokerage firm, they were aware that I knew ASL, Japanese, and Hawaiian but they legally told me I'm not allowed to use any of those. Say a Deaf person were to walk in, I would just have to let them know that I have to call an interpreting service or just not be able to help them at all so that kind of sparked an interest to kind of make ASL a little more available in business services or financial services because in all the offices I've worked in I've never seen anyone who knows ASL or even like just basic things like, "How are you?" Or, "What can I do for you?" Or don't know how to interact with someone who's Deaf.

This idea of incorporating the use of ASL into her practice is noteworthy, but it could also be problematic if she is not fluent and tries to offer help or advice in ASL. I appreciate that Blair recognizes the importance of staying current in the language and 
community, and realizes how her willingness to do so would make a long-lasting impact on the community.

Blair also made another point that I found interesting related to acquiring more than one language. She explained that a friend of hers grew up speaking Spanish and that when he took ASL he found it was much easier for him to learn than for his peers. Blair suggested she believed this was because her friend was familiar with linguistic differences between English and Spanish, and that he could use that information to help scaffold his learning experience of ASL. She noted that because she already knew three other languages (Japanese, Hawaiian, and English) before learning ASL, she felt she had an easier time acquiring ASL than many of her peers.

As we started to conclude the interview, I was curious to know if Blair intended to stay involved in the Deaf community after completing her ASL Studies minor requirements. She stated that she was interested in staying involved and felt she would do so because so many of her closest friends were going into Deaf-related fields. She also shared, "I know that career-wise it's not a focus for me, ASL or the Deaf community, but it's something that I've opened myself up to and I'm interested in learning more about it and being more involved in it."

It is my belief that Blair's intrinsic motivation will be what keeps her moving past LPP and toward the heart of the Deaf community.

\section{Findings Related to Research Questions}

My intention when selecting the three participants for this research study was to explore how second language learners of ASL explained their experience apprenticing 
into the Deaf community. All three participants came from different backgrounds in terms of their exposure to ASL, the number of years they have been learning the language, and their reasons for getting involved in the Deaf community. In this section, I report on my findings relative to my research questions.

Research question 1. How do students in an ASL program at a rural west coast university describe opportunities to engage with native ASL CoPs?

During my analyses of the participants' narratives, I identified two overarching themes in terms of engagement with local Deaf CoPs. The first theme was engagement with opportunities to interact in the Deaf community. The second theme was barriers to engagement with the Deaf community.

Opportunities. All three participants shared emphatically that interaction with Deaf community members is essential when trying to acquire ASL as a second additional language. The opportunities each participant took advantage of and engaged in varied, but the underlying belief that learning language in both the classroom and at events was evident in all their stories. The two main types of interactions that participants believed presented opportunities for engagement were the ASL classes and the ASL/Deaf events. ASL classes. Involvement in ASL classes at WOU was an important factor for all three participants. Specially, Cameron and Blair mentioned the importance of having Deaf/native language models at WOU. All core teachers in the ASL Studies program are Deaf, and participants agreed that having access to Deaf professors at the university, especially during class time, made it easier for them to engage with fluent language models without the added stress of relying on only Deaf events for this exposure. Blair 
stated, "So, I mean in classes, obviously like all our professors are deaf and that class was my first exposure to the deaf community [in Oregon]," and Cameron shared, "I have Deaf teachers . . . so I feel like I definitely have stepped way farther past where I was. I have a lot more one-on-one interactions, I have face-to-face conversations, I have found out more about deaf culture ..."

Blair stressed the importance of having good friends who are also taking ASL classes. According to Blair:

Most of the friends are really good friends that I've made here at WOU have been within the ASL community or within my ASL classes and like the program. So, like I've built friendships through that and I feel like we're a lot closer because, especially at the 300 level of ASL classes, people are in them for a career and they're in them not just because it's for their degree credit so they're a lot more passionate about it and a lot more willing to help each other and support each other in learning.

The connections that students make with one another and the opportunities to practice the language together were positive experiences. Alex and Cameron expressed similar ideas when they shared their interactions with other classmates in the halls, dorms, and on campus.

ASL/Deaf events. Deaf events have been a long-standing requirement for most ASL courses, with the purpose being engagement between native language models and students learning ASL. The benefits of attending Deaf events often were countered by the barriers that exist, making it difficult to attend.

Deaf events range in location, number of participants, purpose, and community base. Sometimes Deaf events are held at coffee shops, restaurants, theater venues, or educational settings. Cameron shared some of the reasons she feels she benefits from attending ASL/Deaf events, "I have deaf events here on campus . . . I have found out 
more about deaf culture and found out about controversial issues that as a hearing person

I would have never realized." She also stated:

I've been to the ASL community event, which was fantastic. I really enjoyed going because there was such a large group of people. I've not gone to a deaf event that is that big, so that was fantastic. The same amount of things that I'm learning at the ASL community event, I learn in class, and it's a very hands-on experience.

Alex agreed that Deaf events are important, but she had a slightly different frame than Cameron. Alex was very aware of her hearing status and student identity when attending events. While she stated she benefited from attending Deaf events, she explained that they were a little more organic. She described her experience:

... having younger people in Deaf events I feel more comfortable, because I can have relatable topics to talk about and stuff. So for me, when I go into a Deaf event, I don't like to just walk up to people and be like, "Hi, I'm this person, I'm a student, let's sign." And it's like, "Okay, well, I'm over here talking to these other people, that's cool. Like glad you're learning, but you know." That's it basically. So I don't like to impose myself in that kind of way. I'd rather it just kind of be like, I'm introduced to that person, we have a few shared interests, and then continue the relationship on from there.

Blair agreed about having events that were less structured and more open to the community. She shared her thoughts about the ASL community event we hosted on campus for the first time right before our interview together. She said:

The [event] that we had this past month that was just like a big group of people from the community coming and just socializing, I think that was probably the most enjoyable Deaf event I've been to. I wish we had more things like that because while I do like ASL club I don't feel like it's a big Deaf event, it's not like we really do much. It's usually the same like four people who sign, the four people who interact or if you go with your friends you only interact with them. So that doesn't feel like a good socializing. I feel like the event that we had last month was probably a better idea.

Since the interviews, the ASL Studies department has hosted three ASL community events and plans to have one more before the end of the academic term. Cameron and 
Alex mentioned they really enjoyed engaging in this type of Deaf/ASL community event on campus.

These two themes also align with situated learning theory. Participants' narratives suggested that having both formal ASL instruction (ASL class) and opportunities for incidental learning (ASL/Deaf events) were equally as important for their language acquisition and their LPP into Deaf CoP.

Barriers. Interestingly, some of the examples of opportunities that encourage engagement between Deaf community members and students (e.g., Deaf events), can also present barriers that makes it difficult for students to apprentice into the Deaf CoP. I noted two main themes in the narratives shared by the participants in my study: acceptance at Deaf events/in the Deaf community, and transportation/resources to attend Deaf events.

Acceptance at Deaf events/in the Deaf community. All three participants expressed apprehension about attending Deaf events for various reasons, and expressed the feeling of taking up space in a community where they were unsure if they belonged. The Deaf community is a minority group, and as such, there is still a desire to have sacred spaces reserved for Deaf community members only. Limiting Deaf events to only Deaf people creates a barrier for students who have to find Deaf events to attend for class credit. Then, the only local option is to attend an event that is not meant to be open to members of the majority culture, specifically not to students who still have emerging language skills. For example, Deaf professionals in Salem host an event termed DNO with the goal of bringing together the Deaf community for a night of conversation and 
fun each month. Due to limited Deaf events in this rural area of Oregon, students started to attend the DNO events that led to several Deaf professionals not attending. These Deaf professionals are teachers, counselors, or other professionals who do not always want to engage with their students or clients during their time off. Cameron shared her experience learning about this sensitive topic during our interview. She disclosed:

Took me a lot of time to understand. I heard one teacher explain to us about the history of having Deaf Club in that it's a place that Deaf people get to meet and they get to communicate and you have that face-to-face prior [time]. And realizing that a lot of time those ASL students do overpopulate that group, so then it turns into an ASL student group where it's all these hearing people and it's no longer a Deaf event. It's a, "I'm here for credit because my teacher told me to," kind of meeting.

While Cameron's focus was on not taking up space, during her interview, Blair seemed to be more concerned about feeling comfortable when attending events. She had some apprehension about attending events because she was nervous about her language skills, and initially she did not have a group of peers to attend with her.

Alex had an interesting experience that she shared about being invited by a Deaf community member to attend a DNO event. She had known this Deaf person for a while and interacted with him regularly, so when he invited her to attend an event with him, she accepted. Her experience did not go as she had imagined it would. Instead, she recounts:

I have gone to a DNO one time. And it was a good time. I know one Deaf person, he invited me out there. So, we hung out. But I noticed it really was just for the Deaf community. It wasn't necessarily something that students were coming in and trying to practice, or anything like that. Which made me feel like I was imposing myself. Like I know I was invited, but I definitely felt like I couldn't just walk up to a person and be like, "Hey, how's it going? I'm just a student, you know, trying to learn and integrate myself." So, I definitely felt kind of out.

Though Alex was invited by someone in the central circle of the Deaf community and had the opportunity to apprentice with him during their times together (old timer 
engaging with the new comer), Alex still sensed that she did not belong at that Deafcentered event. She did not disclose, however, whether her feelings of not being accepted related to her own language fluency or her being one of the only hearing people in a Deaf space.

While Deaf community events present opportunity for engagement, they may also pose sticky cultural and situational barriers that are difficult to navigate for students new to the language and community.

Transportation/resources to attend Deaf events. The other resounding barriers that all three participants experienced were transportation issues and the ability to attend Deaf events not hosted on WOU's campus. All three participants mentioned how difficult it is to find local Deaf events in areas surrounding WOU. They suggested that one must often travel to Salem (30 minutes) or Portland (60 minutes) to find an event to attend. This becomes problematic when a student does not have access to a car or when there is no public transportation to the event the need to attend. To help situate the issue, it is important to note that as of 2019, ride-sharing services (e.g., Lyft, Uber) are unavailable in Monmouth. Bigger towns such as Salem and Corvallis that are within 30 minutes of Monmouth do not have many public transportations options available. Without access to a car, students are dependent on finding a ride with a friend or finding a local event that might be within their community, as was the case with Alex. She shared that in her hometown of Los Angeles, she often found community events she could attend and that the groups she joined were often full of younger people and a more diverse group. Her 
experience with the local Deaf community near WOU was with an older and less diverse crowd.

Alex noted traveling to Portland on the weekends and trying to attend Deaf events, but she lamented that work and school often prevent her from making the trip to Portland, and she misses Deaf events at which she might form stronger connections. Cameron did not have a car when she first came to WOU, so even though her parents no longer decided if she could attend an event or not, she still had to contend with transportation barriers that made it difficult for her to attend events. She was unable to attend any events until she got a car, her second year after coming to WOU. Blair also noted the difficulty of getting to Deaf events, saying:

I'd say for WOU and the ASL classes that it is required to go to deaf events, like at a certain level you have to interact and that's a part of learning the language itself. I thought like your thesis topic was interesting just because I do know that like freshman year it was difficult to get to Deaf events because we're so far away from the city and we're so far away from a lot of these events that are going on. So I think, like in location-wise, it might be difficult to go to bigger events but we have such a strong community here of people who use ASL and such a strong community with the Deaf community as well, so that makes it easier if that makes sense.

Blair did not have any formal exposure to ASL or the Deaf community prior to coming to WOU, so her thoughts about the size and strength of the local Deaf community was dependent on her experience at WOU over the past couple years. Still, she made an astute observation, that while most Deaf events are far away, the local community of Deaf people, mainly the ASL instructors at WOU do bring the Deaf community to WOU's campus.

Research question 2. In what ways do students learning ASL at a rural west coast university feel a part of the Deaf/native ASL CoP? 
A challenge that students encounter when taking ASL as a second language at WOU is the size of the local Deaf community. The size of the community often means that there are not a lot of regularly held Deaf events. While there are pockets of Deaf community members in some of the larger cites around Monmouth, there are not many events available to students for the purpose of incidental learning with native language models. Participants agreed that the available events are welcoming and warm. WOU also serves as its own Deaf community because of the number of Deaf instructors who teach in the ASL Studies program and who engage regularly with students, during class and other less structured interactions. These two themes — warm welcome by the Deaf community and the Deaf community at WOU_-were evident throughout the participants' interviews.

Warm welcome by the Deaf community. All three participants talked about their experiences going to different Deaf events. They made a few references to others who had bad experiences when trying to engage with the Deaf community in other locations, but none of the participants I interviewed had negative experiences at Deaf events. In contrast, both Blair and Alex opened up about their impressions of the local Deaf community. During Blair's interview, she commented on feeling welcome at Deaf events, and stated how this made her more at ease. This feeling of comfort is consistent with the hypothesis proposed by Krashen, relative to affective filter (Krashen, 1982). When a person (student) feels like they can trust the person they are engaging with, in this case, the Deaf community members, they are more open to receiving feedback. If Blair is open to feedback, and the Deaf community members see her intent is to engage in 
relationships, not just satisfy course requirements, they will be more likely to provide feedback and engage with her.

Alex shared about an experience she had at a Deaf event held at the OSD. There was a mix of Deaf, hard of hearing, and hearing people who attended this event, but the prevalent language was ASL. Alex explained:

I would say yes in the fundraiser I felt welcome. I saw a lot of teachers there, interpreting people that I've seen around school, so there was a lot more people that I already felt comfortable with. So going up and speaking to others and meeting even new teachers, like Rian, I met him for the first time there. And it was really nice. It was more comfortable because I already knew the people round, and they were able to introduce me to other people in the community.

What Alex described is the idea of apprenticing into the Deaf community via a CoP. In this example, when Alex met with someone she already knew, who is already part of the Deaf CoP (old timer), she engaged in LPP (Lave \& Wenger, 1991) and was able to establish her place as a newcomer in the community. Alex shared about a few other events, but most of those events were in other locations (her former hometown) that are very different from WOU. Her former hometown was a big city with a very diverse Deaf community, and she was able to find more opportunities to engage with people at Deaf events than here in Monmouth, but she reported still having good experiences at events she has attended here.

Deaf community at WOU. Blair and Cameron both referenced the ASL Studies program at WOU. The program consists of five full-time ASL instructors, and several part-time ASL instructors, all of whom are Deaf. ASL is used in all ASL courses, and Deaf instructors use ASL in classrooms, hallways, bathrooms, the library, and every other area of campus. Five instructors might not be a large number, but participants still felt 
that their strongest connection to the local Deaf community is through their instructors and their ties at WOU. The idea of having a Deaf community nestled in a larger hearing community is not unique to WOU. Many programs have a primarily hearing population, with a subgroup of Deaf community members who live, work, or play in that community. In the case of WOU, all Deaf instructors are actively involved in teaching ASL courses, and participate in events outside of the classroom. For example, often instructors attend sporting events, campus lunch and learn sessions, seminars, plays, and other events. This presence of Deaf people using ASL creates a visibility on campus and exposes students to natural settings where Deaf people engage. Blair told me more about her experience upon arriving to WOU:

So, I mean in classes, obviously like all our professors are Deaf and that [first ASL] class was my first exposure to the Deaf community. So I think, like in location-wise, it might be difficult to go to bigger events but we have such a strong community here of people who use ASL and such a strong connection with the Deaf community as well [through these professors], so that makes it easier [to engage] if that makes sense.

Having her first exposure to the Deaf community come from instructors she met during her first ASL course at WOU, seemed to be favorable for Blair. All five of the full-time Deaf instructors, and many of those who teach part-time, are members of larger Deaf CoPs throughout Oregon, and they actively help students apprentice into those communities as appropriate.

Cameron also shared her experience of engaging with Deaf instructors at WOU. Prior to coming to WOU, she lived in a larger city that had Deaf community members, but the Deaf events were still not easily accessible to her because of transportation. When reflecting upon coming to WOU, Cameron stated: 
All of my previous classes have required me to go [to Deaf events]. And, it took me about a month to realize that it's not required [as much here at WOU] because Western is the Deaf community. I have Deaf teachers; I have Deaf events here on campus, so I feel like I definitely have stepped way farther past where I was [in my ASL skills]. A lot more improvement.

The idea of having easy, consistent, and ongoing access to Deaf community members, via the instructors who teach at WOU, is a motivating factor toward language acquisition. Because there are so many people who use ASL around campus, there is a lot of exposure to incidental learning opportunities that would not otherwise be possible in other communities without a concentration of Deaf professors, and other Deaf professionals on campus. In addition to the five full-time and five part-time ASL instructors, four other Deaf people work full-time at WOU. One person is the program coordinator of the Deaf and Hard of Hearing Educator program. Another is the program coordinator for the Rehabilitation Mental Health Counseling program. The third works as the director for the Regional Resource Center on Deafness, and the fourth works in the Office of Disability Services. There are also many other faculty and staff who know ASL and use it on a regular basis (interpreting instructors, interpreters, Deaf education instructors, office staff, various grant personnel). Having a large number of people who use ASL on a regular basis provides exposure to students that they would not otherwise have if they only had exposure to Deaf people at community events.

Research question 3. What experiences helped students apprentice into the Deaf community?

Participants in this study were not familiar with ways that one might apprentice into Deaf CoPs. In fact, none of the participants had developed plans to seek actively relationship building with members of the local Deaf community. While all three 
participants expressed an interest in continuing their experience in the Deaf community post-graduation, none of them had developed a plan of action for how to make this possible. Apprenticeship into the Deaf community has changed over time. Before the widespread research and teaching of ASL and the introduction of interpreter training/education programs, ASL instruction was person to person. Events at local Deaf clubs brought Deaf community members together, and many states had residential schools for the Deaf where language (ASL), culture, and community flourished. The Deaf clubs served as a meeting place for community members to share news, stories, jokes, and to pass down traditions to new members. Most times, members of the Deaf club were Deaf, but there were times when a Deaf member invited hearing people into the sacred space.

Colloquially, we — members of the Deaf community — called ourselves gatekeepers. We held on to the space that was ours, but when we saw someone who had potential, or showed the right attitude/heart, we would invite him or her into our community. With the advent of technology, Deaf clubs have become less prevalent because people can stay in touch through video calls, email, text, or video messages.

Many states have also closed their residential schools for the Deaf in efforts to send Deaf and hard of hearing students to mainstream programs in their local school districts. As this shift occurred, and people started teaching ASL and interpreting programs opened, there was marked shift in how one apprentices into the community. In the past, the gatekeepers were responsible for brokering this connection, but now that 
students are required to attend events and need to practice, the role of the gatekeeper is no longer as strong as it once was.

This historical frame is important when considering the findings from this research, particularly in regards to participants experience attending Deaf events. Historically, it is important to note that not all locations have large Deaf communities, and even those cities with large communities do not always have many Deaf events. WOU is located in a small town without a large Deaf community nearby. Having a small community limits the number of Deaf events that are feasible for students to attend, and often necessitates that students travel 60 miles or more one-way to find an event. All three participants in my study shared the challenges of finding and attending Deaf events here in Oregon.

Deaf events. As noted previously, Alex came from a large city that had many very active Deaf communities, so she was able to engage with Deaf people easily in her hometown. She explained that even though she had many opportunities, she still often felt a barrier to participation because she was an ASL student, rather than someone who wanted to engage in the community. To combat this, she found smaller groups of Deaf people who enjoyed similar activities as she did, and often spent her time in those communities. She felt this organic and authentic interaction was key to her involvement in the Deaf community in California. After moving to WOU, she shared that she had much less direct interaction with Deaf people or Deaf communities here, because most of the local Deaf community members are older and have different interests. This lack of authentic connection with Deaf community members has made it difficult for her to 
continue apprenticing into the Deaf community since arriving to WOU. She stated that she believes she will be able to find a community in Oregon, but that she recognizes that she will need to extend herself to find one that is a good match for her.

Cameron also had experience interacting with Deaf communities before coming to WOU, and shared a similar sentiment as Alex, about how it is difficult to shake the stigma of being an ASL student when attending events. Even though she did not attend the events just for credit, many of her interactions and those of her friends were awkward because she was a student. This initial awkwardness did not stop Cameron from attending events, and actually was one of the reasons she was inclined to apply to Gallaudet University so she could have a more immersive experience in the Deaf community. When she researched interpreting programs, she learned about WOU, and after visiting both WOU and Gallaudet, she decided that WOU would be the best fit. In contrast to Alex, Cameron shared that she feels more connected to the Deaf community here in Oregon, than her home community in Washington did. Cameron explained:

As a student it's actually quite nice [to have a Deaf community at WOU], I was commuting so much before, so in a way I feel a little lazy because I just have to be here. But it's fantastic because I've also had the opportunity to expand [my experience] to the DeafBlind community which I realize now was very large in Seattle and that's where I was going for my Deaf events, but didn't know about it. And so, now I'm realizing more about these communities, that as a hearing person I was unaware of.

Cameron also told me that her great-aunt is an interpreter in Washington, so she had some initial reservations about how to engage in the community. Her aunt did not attend an interpreter training/preparation program, and many of her comments left Cameron a little leery. When prompted, she elaborated: 
... [my aunt] had expressed to me all of these issues I would have with getting involved into the community and so I very much was concerned about transferring to Western if other people had met her, my last my name, because I know how much her name means in the Deaf community. I definitely have been more worried than I've actually experienced.

This is an interesting point that surfaced during the interview, because as Cameron mentioned, reputations are important in the Deaf community. Often times, when a Deaf person is impressed with an interpreter/student/person learning ASL, they share that knowledge with others in the community; then, the Deaf community accepts that person. By contrast, if a Deaf person has a negative interaction with an interpreter/student/person learning ASL, the Deaf community also shares this knowledge with others. This negative interaction could be the person's skill level or stem from the person's attitude. Cameron's concern about some backlash in the community because of her aunt's reputation is not unrealistic; yet, I have often found that Deaf community members often give people the benefit of the doubt, and genuinely hope that those who want to become part of the community are doing so with open minds and hearts. I was pleased to learn that Cameron had not experienced any negative consequences or encounters in our community at WOU.

Blair's circumstances were different, because she had not engaged with members of the Deaf community, or attended any Deaf events, before coming to WOU. She enrolled in her first ASL class, and it was difficult for her to attend required events because she had not made many friends and did not have a car her first year at WOU. By her second year, she connected with other students in her classes, and the deeper those connections grew, the safer she felt attending Deaf events with her group of peers. Now, even though her professional career is not in a Deaf field, she is committed to staying 
involved in the Deaf community and interacting with her friends who are going into careers that will make them active members of the community (e.g., interpreters, educators).

Based on feedback from WOU students about the difficulty in finding Deaf events to attend, the ASL Studies department committed to hosting ASL/Deaf events on campus beginning January 2019. The department designed these events to be at WOU, open to the public, and a way to flip the frame of the traditional course requirement of attendance at a Deaf event in the community. The intention of these WOU events was to draw the Deaf community to campus, provide community members with opportunity to share ASL stories, lead games, and engage with students learning ASL. The intentionality behind this flipped frame was to take the stigma away from students who felt their presence at Deaf events was not welcomed, and to also allow more Deaf events to stay centered in Deaf space. Prior to these interviews for this study, WOU hosted one ASL/Deaf event on campus and had more than 70 students and 10 Deaf community members in attendance. Both Cameron and Blair attended the first ASL/Deaf event on campus before completing their interview with me.

\section{Limitations of Study}

A qualitative study, specifically a case study design, was an effective and appropriate way to capture the experiences and stories of the three participants in my research study. A case study is a research method for an empirical inquiry that explores a phenomenon within its real-life context. A case study is a descriptive and exploratory analysis of a person, group, or event. In my case study, the case is the phenomenon of 
becoming a member of a Deaf CoP. Through my cross-case analysis, I noted similarities among three individual cases. I contend that the findings from this study will not only inform the teaching practices at WOU, but may also help to guide future research. Nevertheless, the small sample size and interview data set were limitations of my case study

Another limitation to this study was the lack of research examining how a secondlanguage learner apprentices into the Deaf community. The majority of my literature review was comprised of research concentrated on hearing communities. At a 2018 ASL Studies conference held in Washington, DC, the consensus among participants and presenters was the need for an increase in the research focused on ASL Studies as well as an increase in the number of Deaf people who conduct the research. My research study may contribute, even in a small way, toward addressing this gaping hole in the research of our field.

An additional limitation I want to acknowledge is that WOU is not representative of all universities that have ASL Studies programs. While WOU has a large ASL Studies program and its students come from many different backgrounds in terms of how they first acquired access to ASL, WOU is not a very diverse campus. All the students who agreed to participate in my study, and the three participants I chose ultimately to participate in my research, were similar in terms of demographics (race/ethnicity, gender, social class, hearing/hard of hearing/Deaf/DeafBlind). The lack of diversity in my sample may have had an influence on the experiences students had while trying to move through LPP and find apprenticeship opportunities into Deaf CoP. 
While I hesitate to consider my positionality as a Deaf researcher to be a limitation, I have to take into consideration that participants might have answered some of the questions in a particular way because I am Deaf and a member of the Deaf community. All three participants have an interest in continued involvement in the Deaf community, so they might have been more careful when answering some of the questions than they would have been if the interviewer/researcher was a hearing individual. I did my best to assure participants that I wanted their honest answers and that I respected their experiences. It might also be possible that I possess some internal biases as a Deaf person that I am unaware of at this time. Notably, I tried to be mindful of my biases, ground my assertions in research, and check with others when making decisions about research design, questions, and implications.

Despite these limitations, I believe the stories shared in this dissertation are a valuable snapshot of the current trends facing the ASL/Deaf community in terms of how hearing students are acquiring ASL as a second language, and the ways that access to native language models (or lack thereof) plays a role in this acquisition process. 


\section{Chapter 5: Conclusion}

The ASL Studies program at WOU is foundational in the preparation of students who are interested in being part of the Deaf community, but direct classroom instruction is not enough. Access to native language models is a key component to language acquisition, but WOU is located in a small rural area that does not have a large Deaf community. Lack of exposure to native language models directly impacts the problem of practice we face at WOU. The problem of practice is that students often have little exposure to rich language models who are fluent in ASL, which impacts their LPP in the local Deaf CoPs and reduces apprenticeship opportunities that might be beneficial for their language acquisition experiences. All three participants in my study were interested in being part of the Deaf community and building relationships with Deaf community members, but felt limited in their ability to make this happen, due to lack of opportunity and the presence of barriers.

The following research questions guided my study:

1. How do students in an ASL program at a rural west coast university describe opportunities to engage with native ASL CoPs?

2. In what ways do students learning ASL at a rural west coast university feel a part of the Deaf/native ASL CoP?

3. What experiences helped students apprentice into the Deaf community?

It was not an easy task to decide on these research questions. Thankfully, my colleagues at WOU and my instructors at PSU helped me develop questions that got to the heart of what I wanted to learn. In 2018, I ran a pilot study with students in preparation for this 
dissertation research. My questions were much less defined, and the data I collected from that study, addressed students' ASL skills and their experience in the third year ASL courses at WOU. At the conclusion of the pilot study, I realized that I was less interested in fluency (as measured by a pre and post assessment), and more interested in students' experiences and emotions relative to connecting with members of the Deaf community.

Throughout my research over the past three years, it became even more apparent that there is a need for language opportunities for students, both in the classroom and with members of the Deaf community. Learning a language only in the classroom, without opportunity to engage in incidental learning (Deaf events or engaging outside of the classroom) is contrary to the research presented in situated learning theory (Lave \& Wenger, 1991). The need for both types of learning was a main theme that became apparent throughout my study of the literature and in my own research. Additionally, the idea of engaging with members of the Deaf community is important because this allows students to engage in LPP as they work toward connecting with members of Deaf CoPs. Deaf instructors at WOU, and other local Deaf community members, provide apprenticeship opportunities for students that foster language development and help students acquire ASL and knowledge of the Deaf community and culture. In this way, the Deaf instructors at WOU serve in the role of gatekeepers to the larger Deaf community. This role is limited, because ultimately students could be admitted into the program even when they lack the appropriate skills, given the underlying need to keep the ASL Studies program growing. As mentioned in a previous chapter, the role of Deaf community members as gatekeepers has become almost extinct, with the institutionalization of 
teaching ASL. The Deaf instructors at WOU, and I would venture some other programs across the United States, are still trying to hold tight to the gatekeeper role to protect Deaf community members, Deaf culture, and ASL.

I found two themes, varied learning environments and CoP/apprenticeship, continually reinforced in my interpretation of the data and analysis of my findings. The first theme, the importance of situated learning, revealed the need for students to be actively engaged in two types of learning: explicit instruction and incidental learning. The second theme, CoP, provided a good rationale for hosting more ASL events at WOU, bringing in Deaf community members to engage with students. Fortunately, the instructors who teach in the ASL Studies program are Deaf and work to establish a thriving Deaf community at WOU. With nine full-time Deaf professionals who work on campus, WOU has a critical mass of people who use ASL, and this allows students to have opportunities to engage with members of the Deaf community, without having to travel to events. Increasing opportunities for engagement, while decreasing barriers, was the key outcome I hoped to address with my dissertation research.

\section{Synthesis of the Findings}

In this section, I offer a synthesis of the two major findings of my study: varied learning environments and $\mathrm{CoP}$. With regard to varied learning, I specify the importance of explicit instruction and incidental learning. Then, I discuss the critical nature that CoP plays in second language acquisition of ASL.

Varied learning environments. Students can learn in a variety of environments. These varied learning environments make it possible for students to learn different types 
of ASL that they might not otherwise had exposure (e.g., medical jargon, sports terminology, teenage slang). These environments are central to the success of ASL students because they include a balance of explicit instruction by qualified instructors and incidental learning by engagement in a CoP.

Direct/explicit instruction. Learning ASL in the classroom provides a foundation for students to learn more about what makes ASL a distinct language separate from English. It also provides an opportunity for students to learn more about Deaf history, culture, and the Deaf community. Classroom instruction allows course content to be developed, revised, added, deleted, repeated, or thrown out, depending on how students are progressing in their lessons. In other words, instructors frame their classroom instruction to respond to the specific needs of their students. As of 2019, only a handful of reputable ASL curriculum resources are available for use by high schools and colleges/universities across the United States. Instructors often need to supplement the curriculum materials with their own ideas, activities, and assessments, which means that each course varies a little. The benefit of having multiple Deaf instructors at WOU is that each time instructors teach the content, students are exposed to new nuances of the language, regional dialect, and other intricacies of the language and culture.

All three participants in my study experienced a combination of Deaf instructors at WOU. While we did not talk about the differences among teaching styles or why they chose to take various instructors, rather than staying with the same instructor (if their schedule permitted), I wish we had. I think it would have been interesting to explore if students could identify differences in teaching style, content knowledge, language 
fluency, or adherence to Deaf cultural norms of various Deaf instructors, but that was beyond the scope of this study. For this study, all three participants spoke of the value of having all Deaf instructors in their ASL courses here at WOU, and stated that the Deaf instructors/faculty/staff at WOU formed their own mini community in an area lacking other larger Deaf community groups.

By the time students complete ASL 9 at WOU, the last course in the three-year ASL core course sequence, they have had over 360 hours of direct ASL instruction. This number does not include any ASL elective courses they might have taken in addition to their required coursework. This is a large number of hours spent learning ASL, but the need for interaction with Deaf community members and incidental learning is still imperative (Lave \& Wenger, 1991).

Taking an ASL course is sometimes the first interaction or engagement a hearing person has with a member of the Deaf community. This was Alex's experience as she shared:

Honestly, when I first even heard about the Deaf community, I was surprised that I didn't even think about that community in America. And I love learning about different languages and cultures, so that's really why I want to immerse myself into it, so I can become a better signer, and really actually get to know this culture that is basically in our own back yard and had no idea it had even existed before I took a class.

When I asked about her what she hoped to learn at the completion of her ASL courses at WOU, she added:

I would say, after completing [my degree] here at Western, I would like to have basically the confidence through my ASL learning through all my classes, my language [skills], and learning more about the culture, and the community. 
Alex had exposure to other ASL classroom environments before coming to WOU, but she still felt that she wanted to learn more about language, culture, and community. This seems to suggest that even after taking three to five years of ASL courses, confidence in language production likely comes from repeated engagement with native language users.

Blair also talked about her experience learning ASL in the classroom. With no prior exposure to the Deaf community or ASL courses before coming to WOU, her lens was unique as compared to other the other two participants. According to Blair:

... most of the really good friends that I've made here at WOU have been within ... my ASL classes and the program. So, like I've built friendships through that and I feel like we're a lot closer because, especially at the 300 level of ASL classes, people are in them for a career and they're in them not just because it's for their degree credit, so they're a lot more passionate about it and a lot more willing to help each other and support each other in learning.

Blair is correct - most students who persist through the third year of ASL coursework intend to go on to a graduate program or work in a field related to the Deaf community. Students who take ASL simply to satisfy their language requirement (stop after the second year), may have very different views on the importance of direct instruction in the classroom as opposed to incidental learning from community members.

Hearing instructors. Hearing people teaching ASL is a sensitive subject for many of us in the Deaf community. While there are hearing teachers who are fluent in ASL, taking a position teaching ASL in some cases means taking an employment opportunity from a Deaf person. Many jobs require licensure, certification, or an advanced degree to teach, and to qualify for these credentials, one must have a certain command of higher order English. For a person who is Deaf, raised with ASL as their first language and English as their second language, this lack of fluency in English often poses a barrier to 
attaining employment and presents an unfair advantage to hearing peers who were raised with English as their first language, and who can more readily pass the tests for these credentials. While it is important to have a good command of English, having this be the prerequisite to being able to teach ASL, perpetuates the belief that English is a superior language and one must be fluent in English to be qualified to teach ASL. For many years, professionals thought the best way to help the Deaf community was to make us more like the hearing majority, and this meant giving preference to English over ASL (Jankowski, 1997).

This frame is troublesome, because a hearing student could study ASL for 2 years, graduate from college with a Bachelor of Arts degree, pass a teacher licensure exam and get a job teaching ASL with only two years of the language. In contrast, a Deaf person raised with ASL as their first language, is part of the Deaf community, ascribes to Deaf culture, but struggles to pass a test that often measures English mechanics and understanding of trick questions, not actual content, might be turned away from that same position and be deemed not qualified. This is not to say that all Deaf people are qualified to teach ASL, nor does it suggest that no hearing people should teach ASL. It is my stance that there are some exceptional circumstances where hearing people have aligned themselves with the Deaf community, and after mentoring, apprenticing, and assimilating into the Deaf community, those people make wonderful allies and teachers. That said, it is still my belief that, in general, Deaf people who are fluent in ASL, should be teaching ASL courses when possible. 
Historically, the majority culture oppressed Deaf people, and this oppression led to fewer opportunities for employment. Specifically, Houston (2018) identified four main themes of oppression: systemic factors, difference, elitism, and success and barriers. Due to this oppression, many Deaf people have missed opportunities to teach or obtain other gainful employment. It is important to note, the converse is also true. Houston found that when Deaf educators had the appropriate supports in place (e.g., interpreters, support systems, working relationships with coworkers) they reported feeling more able to succeed in their work environments. If Deaf people had the appropriate supports, perhaps there would be fewer hearing people teaching ASL, because more Deaf people would be qualified to fill those positions.

Before coming to WOU, both Alex and Cameron each took at least one ASL course with a hearing instructor, and felt that it benefited them because the instructor was a good model of a hearing person who was part of the Deaf community, but they both agreed that it was not the same experience as having a Deaf instructor. Alex's experience learning from a hearing instructor was a positive one. The instructor provided a good example of how hearing people can be allies for our community, by encouraging students to learn our language and be active in our community. Our community is always grateful when we learn about hearing allies who instill the importance of engagement with Deaf community members.

Incidental learning. Engaging with members of the Deaf community is an essential component to learning ASL (McKee \& McKee, 1992). This concept is grounded in research that predates the instruction of ASL for course credit. Both social 
interactionist theory and social interactionalist theory support the emersion-based ASL Studies Program offered at WOU. Social interactionalist theory focuses on building relationships and being in community with other language users and this is a fundamental reason our program requires students to engage in Deaf events and participate in incidental learning opportunities. In contrast to direct instruction, incidental learning takes place in informal settings and through unplanned interactions (DeafTEC) with others. Specific to second language acquisition for ASL students, incidental learning occurs when hearing students engage with Deaf native language models outside of the classroom environment.

Incidental learning occurs in a myriad of ways. At WOU, our ASL instructors often attend sporting events, celebrations (e.g., Martin Luther King Day of Service and commemorative banquet, Coming Out Monologues), plays, ASL Club, and other events on campus. These types of opportunities to engage with native language models in informal settings and environments provide students with the opportunity to learn through interaction and start to build relationships outside of the classroom. These relationships lead to increased trust and a sense of connection that can help students be more apt to accept feedback about their language skills. Krashen (1988) spoke of this process in his fifth hypothesis, Affective Filter. In this hypothesis, the higher the filter, the less likely a student is to take feedback or correction from the person with whom they are engaging. The reverse is also true, the lower the filter, the more likely a student is to take feedback or correction. A person's affective filter is influenced by their psychological response to a given situation. Students' affective filter in the classroom is 
generally lower once they become familiar with their instructor's teaching style, requirements, and expectations. Lowering an affective filter in incidental learning situations can also occur, but it can be difficult depending on the situation. For example, if a student attends a Deaf event and meets a Deaf person one time, and does not have a relationship with that person, according the Krashen's hypothesis, the student will have a higher affective filter and will be less likely to be receptive to feedback. In contrast to this scenario, however, imagine a student attends a Deaf event and immediately connects with a Deaf person and they continue to meet up for coffee or meet at other Deaf events. As they develop a relationship with this person and establish rapport, they will be more likely to accept feedback relative to their language skills from this person.

One of the advantages of hosting ASL/Deaf community events at WOU is that so many of the WOU Deaf instructors and staff attend these events. This means that students have opportunities to engage with the WOU Deaf community both in the classroom (direct/explicit instruction) and at these informal events (i.e., incidental experience). One important factor for language acquisition is access to fluent language models (Krashen, 1988; Vygotsky, 1978). In May 2019, WOU hosted its fourth event of the year with a total of 65 students and 25 Deaf people (five WOU instructors) in attendance. Many students approached me after this event and asked if the community nights would continue fall term. These students shared that they believed these events were powerful experiences and that they could not wait for the next academic year, so they could attend more of them. Their anecdotal feedback confirmed for the ASL Studies team that 
informal models (i.e., incidental experience) work for our students, and we are committed to continuing to host these events ongoing at WOU.

The most exciting part about these informal events at WOU is that it flips the frame on the concept of students attending Deaf events. In the old frame, students (e.g., participants in this study, other ASL students) expressed feelings unwelcome because they were going into a community event that was not designed for them. In this new frame, the informal ASL/Deaf events at WOU were designed intentionally to allow for students to develop relationships with the Deaf community. Deaf community members are invited to attend these events with the understanding that they are doing so to help foster students' language acquisition. When students know that Deaf community members are there to support them, this could help lower their affective filter and allow them to have more interaction and engagement without the fear of judgement.

Knowing how students feel about these events is important, but we as instructors also need to consider the experience of Deaf community members to ensure they are benefiting from these events and interactions with students as well. Under the old frame, where students are encouraged to attend Deaf events to engage with Deaf community members, there is not always equal benefit for both parties (Deaf person and hearing student). The goal of this flipped frame ASL/Deaf event is to encourage reciprocity between students and community members, so all benefit from their interaction together. It is important to note, Deaf people are not simply tokens at these events. They play key roles in planning the event, leading games and activities, and engaging with students. One of the most exciting partnerships that developed from these community events is with the 
OSD, specifically, their Adult Transition Program. Deaf students from the Adult Transition Program, and their instructors, have attended all of our monthly events. One of their instructors recently shared with me that students look forward to these events and have already asked about the ability to attend again in the fall.

Bringing Deaf people to WOU's campus has so many benefits beyond simply serving as native language models for ASL students. These events provide real opportunities for students and Deaf community members to engage in relationship building and connection, which are precursors to apprenticeship into more intimate parts of the Deaf community. As students continue to engage with Deaf community members, they have the opportunity to move pastLPP, and into more meaningful relationships as active participants in Deaf CoPs. While these events provide great exposure and an opportunity for people to learn about ASL and the Deaf community, in terms of developing language fluency and second language acquisition, students must engage with native language users for this process to be effective (Krashen, 1988; Vygotsky, 1978). This means that simply attending the events each month is not enough; students must be actively engaged to benefit from their attendance.

CoPs. Situated learning and CoPs (Lave \& Wenger, 2001) are theoretical frames that provided me critical new lenses with which to view students' assimilation process into the Deaf community. I have taught ASL for 15 years, and prior to my doctoral work at Portland State University, I was not aware of these fields of research. As noted previously, the importance of situated learning is essential for second language acquisition. The significance of native language models, particularly those who are 
willing to provide feedback and quality language input (Krashen citation) cannot be overstated.

CoPs come in many different shapes and sizes. Not all CoPs share a similar structure, number of participants, goals for the community, or types of activity. Some CoPs are formal, while others are more informal. The Deaf community is no exception. There are many different intersectionalities within the Deaf community, and therefore, many different types of Deaf CoPs (e.g., educators, artists, actors, runners, writers, Deaf people of color, Deaf LGBTQ members). When I first started my research and came across the idea of CoPs, I could not find any research that applied this frame to the Deaf community. I was curious if the participants in my study were familiar with the concept of CoPs. Blair shared:

I've learned about it in some education classes and from my mom as well because she works in the DOE, Department of Education, so the concept of learning with a group of people who are interested in the same thing but I don't have like specific research or any knowledge of it.

I was impressed that Blair had an underlying idea of what CoP meant, and I found that it was easy to engage with her about this topic because she already had a prior understanding the most basic meaning of a CoP meant that she was able to transfer that knowledge and apply it to her positionality within a Deaf CoP.

In contrast, Cameron shared that she was unfamiliar with the concept of CoPs, though she tried to make an informed guess about its meaning. She said:

Not sure if it's culture-based or language-based, because if you're a CoP of a language, then meaning the language is continuing generationally and that it's progressing and you're getting more vocabulary. Or is it culturally where you're continuing a culture where you're spreading the knowledge? I guess I don't know what kind of practice. 
I loved that Cameron felt safe enough to explore possible ideas of what the term CoP meant and that she was able to come up with two thoughts, one related to language and the other to culture. CoPs definitely have elements of both language and culture, so in this way, she was not entirely off base in her thoughts. This is especially true for Deaf, because as a minority group with a shared language, preservation of ASL is one of our top priorities.

Similar to Cameroon, Alex was not familiar with the term CoP; however, she also grasped its meaning based on the context of our conversation. She offered:

I can't say that I've heard that exact phrase, but I definitely think I have the idea of where you know, you're always pushed to be active with going to the Deaf events, or meeting other Deaf people to sign in real life conversations, not just formal school settings.

Similar to the other two participants, Alex had a vague understanding of what CoP meant and how it related to her experience in the Deaf community. When combining all three of their definitions, it captures the essence of a CoP: group of people learning together (Blair), through language and culture (Cameron), through real life conversations (Alex). I think it is fascinating that each of the participants keyed in on a different component of a CoP. When I talk about CoPs in the future, I will start with this co-constructed definition because it captures the heart of how I use CoP in my research about students connecting with members of the Deaf community.

How people gain admittance into these communities varies, so there is not a clear path one must follow to be accepted. As hearing students learn ASL and become interested in the Deaf community, trying to find a CoP that is of interest to them can be 
difficult. Initially, they might seek to find a CoP of hearing peers because they feel more comfortable.

Sometimes when they do identify a community they want to be part of, it becomes clear that the community mainly consists of Deaf people. Gaining admittance into the community requires an invitation from one of the community members - an apprenticeship. A student interested in acquiring language will likely be more successful in their language acquisition if they interact with native language models, and if they engage in both explicit and incidental learning. Becoming involved in a Deaf CoP is one way to increase opportunities to use ASL skills and receive feedback from community members.

Apprenticeship opportunities at WOU range from formal (program requirements) to informal. Some students are required to complete internships that necessitate their involvement in Deaf CoPs (e.g., student teaching at the residential school for the Deaf), while others choose to get involved in Deaf CoPs because they want to form lasting connections and relationships (e.g., church, sports, music). Finding an apprentice is not always easy, because it requires commitment on the part of the student and the Deaf community member. When the apprenticeship is part of a formal arrangement (e.g., student teacher paired with a supervising Deaf teacher), there might be more consistency in terms of involvement, but less authentic connection.

By engaging in this case study research, I surmised a few additional findings. First, diversity (e.g., age, race, gender, interests) matters. This seems like a simple enough concept, but I had never considered how diversity might play a big role in a 
student's motivation to continue to learn more about the language and culture of the Deaf community. As a Deaf person who teaches ASL, I do not often have the option to engage only with people with who I feel an authentic connection. People look to me for feedback on their language or their engagement in the Deaf community. I have to make an effort to connect with everyone, so learning that these three participants engaged more when they found Deaf individuals with who they had an authentic connection was a little surprising to me.

The location of the Deaf events also seemed to play a role in participants' ability and/or willingness to further engage in local Deaf CoPs. For example, if the event took place at a bar or location that only admits patrons who are over 21 , this automatically excludes students and community members who are under 21. Additionally, if a student has an aversion to alcohol for some reason (e.g., religion, sobriety, stigma), even if they are of age, they might not choose to attend this event. Another factor that comes into play is finances. If a student is of age, and has no problem with attending an event at a bar, there still is a possibility that the cost might be prohibitive. These privileges (e.g., preferential connection, age, financial means/social class) were not among the factors I considered prior to my research and interviews with my three participants.

\section{Implications}

Research on second language acquisition makes it clear that exposure to native language models is essential (Krashen, 1988; Lave \& Wenger, 1991). This is challenging for rural areas that do not have large communities where students can easily find events to attend or connect with an apprentice to help guide their journey into the community. 
This is particularly true for students at WOU, who need to engage with members of the Deaf community, but often have a difficult time finding events that are appropriate for them to attend. This has been a long-standing challenge for students in our Deaf studies program, and while we have known about this for many years, it was not until recently that we started to flip the frame on community engagement.

The three participants in my study all experienced challenges and barriers when trying to locate Deaf events in Oregon to satisfy their class assignment/requirements. Transportation, distance/location, age of local community members, and feeling nervous about meeting new people, were all common experiences participants faced after coming to WOU. Historically at WOU, there have always been Deaf instructors in the ASL Studies program, and the number of Deaf students fluctuates from year to year. Even though this is the case, WOU did not have any structured events designed to bring the Deaf community and hearing student community together for the purpose of language and connection.

The idea to hold monthly events on campus came from a presentation by Thomas Holcomb at the ASL Teachers Association conference in 2017. After pondering this idea for a bit, I reached out to other Deaf community leaders and asked how they were ensuring students had opportunities to engage with their local Deaf CoPs. Following this conversation, the idea of hosting monthly ASL/Deaf events at WOU was born. Initially, the idea prompted some apprehension and resistance, because it meant that our ASL faculty at WOU would need to put in more time and work with ASL students in addition 
to their classroom hours. Once the faculty realized that there was no requirement for them to attend, the initial reluctance dissipated.

From the very beginning, students were excited about the idea of having an event on campus that brought the Deaf community to them, and that challenged the traditional frame that felt so restricting and difficult to navigate. The first event of the year included 75 students and five Deaf community members (WOU instructors). The second event, cohosted with the local DeafBlind community, drew more than 100 students, and 23 Deaf/DeafBlind community members (including WOU instructors). The third event occurred right after spring break, and 55 students and 8 Deaf community members attended. The final event of this year consisted of 75 students and 25 Deaf community members. Long after the ending time of the event, people were still milling about connecting with one another and using ASL. It was bittersweet to know that this event was the last one for many of the senior students who would not be returning to WOU in the fall.

As students left the final event, many stopped by and asked me about plans to continue these events next academic year. I assured them that this new flipped community experience would continue fall 2019, and students were relieved and excited to get this information. Many Deaf community members also asked about plans to continue these events because they enjoyed the opportunity to engage and connect with students at WOU.

Perhaps one of my favorite unintended outcomes of these events was our new connection with the OSD, in particular, their Adult Transition Program. Students from 
OSD are learning valuable new skills by attending our events. Our WOU ASL students get to engage with native language models, and OSD (Deaf) students, have the opportunity to learn how to engage with hearing people who are not fluent in ASL. In this way, these events provide growth opportunity for both communities. One of the challenges in recent years has been to recruit Deaf students to attend WOU. At one time, nearly 15 years ago, there was a large student body of Deaf students at WOU. As of 2019, there are only a handful of Deaf and hard of hearing students at WOU. By bringing in Deaf community members, especially those Deaf youth who attend the OSD Adult Transition Program, we expose new generations of Deaf people to WOU, and we hope this will lead to an increased number of Deaf students who enroll at WOU. An increased number of Deaf students, whether from OSD or other surrounding schools, would provide more native language models for our students to engage with on an ongoing basis, and could lead to more authentic apprenticeship opportunities for those who are interested.

At WOU, many of the students who complete the third year of ASL coursework, go on to complete graduate programs in Deaf related fields, or work in Deaf related employment settings (e.g., interpreting, teaching, mental health counseling, vocational rehabilitation). This desire to continue in a field using ASL makes it even more essential for students to have as many opportunities as possible to engage in Deaf CoPs and apprenticeship. Not only could this lead to more opportunities for language acquisition, it could also provide students with stronger ties to the Deaf community and prepare them to be better allies in our community. Additionally, the more people who are fluent in ASL at 
WOU and in the surrounding areas, the more opportunities there will be for Deaf community members to engage in activities and events that might have historically not been accessible to them. As we continue to build connections and networks of CoPs (Deaf and hearing), both communities are likely to experience benefits from the increased connection.

I would like to share an example of community growth here in the towns surrounding WOU. In most of the coffee shops, grocery stores, restaurants, and bars, at least one employee knows ASL. I can often walk in to one of these places and engage, even if just peripherally, with someone who knows a little bit of ASL. This makes a huge difference to me, personally, because it is an indicator of human connection, through language, that makes me feel more engaged and accepted in our local community. It also has an impact on me professionally, because I know the work we are doing in the ASL studies program is working. Students learn ASL and are confident enough to use their skills to engage with Deaf community members.

\section{Future Considerations and Studies}

My opportunity to engage in research that directly addressed the problem of practice faced by ASL students at WOU led to a reframing of required attendance at Deaf events. While instructors will still encourage students to look for opportunities to engage with various Deaf CoPs, students will have increased capacity to attend and engage in LPP given the monthly ASL/Deaf community events. The ASL Studies team has hosted four well-attended events thus far, so there is a lot of anticipation from students and community members for these events to continue. 


\section{Research in Other Rural Communities}

The findings of my research study have implications for next steps to take as a community at WOU to increase language acquisition opportunities between hearing students and local Deaf community members. My sample population was small, with only three participants. Therefore, I am unable to generalize the results of my study and assume that other rural communities face the same barriers identified in my study. I also do not want to assume that other institutions would be able to replicate the ASL/Deaf community events on their campuses. Nevertheless, I would like to continue my research about LPP and apprenticeship in the Deaf community. For example, I could conduct research with other rural programs to see if their students and Deaf community members have encountered similar opportunities and barriers as those students at WOU face. In addition, in the next round of research, I would like to include participants from community colleges, technical/training programs, private college/universities, and community groups.

If the results of that research show that other communities experience the same types of barriers, this data would be a good indicator that a national task force should be established to address the needs of second language learners, and how local Deaf CoPs might be able to support language instruction and acquisition in more incidental learning environments.

I would also like to conduct similar research on programs that have large Deaf communities that are more actively involved in their program. I would like to examine compare the data from rural locations (with limited access to Deaf CoPs) with that of 
programs that have a larger and stronger Deaf presence. I am curious to see if the attitudes, opportunities, and barriers disclosed by participants in my study would be the similar for students in in programs with a larger Deaf presence.

Additionally, I want to do an ethnographic study with Deaf community members about their experience engaging with hearing students who are interested in becoming part of the Deaf CoP. I believe it is important to not only gather feedback and document the experience of students who are trying to apprentice into Deaf CoPs, but also consider the experiences of Deaf people who are dealing continually with members of the majority culture (i.e., hearing people) who are trying to gain access to their community. Having narratives from both communities is an important next step in my continued research on this topic.

DeafBlind communities and protactile language. I would also like to broaden my research to include members of the DeafBlind community. As I mentioned earlier, local DeafBlind community members cohosted one of our ASL/Deaf events. In September of 2018, Jelica Nuccio, one of the national leaders of the Protactile Movement within the DeafBlind community, moved to Monmouth, Oregon. Protactile is a general term that encompasses the history, attitude, language, and philosophy of the DeafBlind community. Protactile language is an emerging language - one that is separate and distinct from ASL.

Jelica runs a training and education center (Tactile Communications, LCC) and works at WOU on a federal grant project, and teaches in the ASL Studies department. Jelica recently taught the first DeafBlind Studies course in protactile language, a 
historical event because no one has taught such a course. Jelica is interested in working with the ASL Studies team to develop additional courses in the field of DeafBlind Studies and protactile language. We recognize students' who are interested in learning protactile language, and working in DeafBlind communities, will need opportunity to engage with local DeafBlind CoPs. Prior to Jelica's move to WOU, there was not a sizable or active DeafBlind community in the area. Now that Jelica's training center is located close to campus, there is an increased number of DeafBlind community members and various DeafBlind CoPs (e.g., gardening, woodworking, protactile theater, cooking).

Having a growing DeafBlind community close to WOU is important, because just as the ASL Studies program encourages students to engage with native language users, students who wish to learn protactile language will also need to engage with native protactile language users. Protactile language is an emerging language that is separate and distinct from visual ASL, and the types of events that students attend for exposure to protactile language will be different from those for visual ASL. The first time Jelica and I cohosted an ASL/Protactile event at WOU was a good experience, but we need to consider many barriers and opportunities before hosting additional events. ASL is a visual language, and protactile language is a tactile language, so how one must communicate is different. Setting up clear expectations for students on how to adhere to Deaf and/or DeafBlind social, cultural, and linguistic norms, will be essential as we move forward. To our knowledge, no other events combine both communities and promote the use of both a visual and a tactile language. More research and exploration is needed to identify how best to run events that combine both communities and languages, without 
negating the autonomy of either community. At WOU, a large number of students want to learn either ASL and protactile language, so having events, whether combined or separate that bring in both communities (Deaf and DeafBlind) will provide students with incidental learning opportunities to engage in second/third language acquisition.

Recruitment of Deaf students. Another important consideration is how to recruit more Deaf students to attend WOU. According to Cawthon, Garberoglio, and Bond (2016), unemployment rates are higher in the Deaf community than in the hearing community. Currently, the employment rates are uneven with $72 \%$ of hearing people employed and only $48 \%$ of Deaf people employed. Systemic issues related to access might also contribute to this discrepancy (e.g., unqualified interpreters in the classroom, at job interviews, in training programs). Other possible contributing factors might be lack of allies (e.g., less hearing people apprenticing into Deaf CoPs), language barriers, and educational systems that put Deaf students at a disadvantage. Increasing the number of Deaf students at WOU has the potential to increase the number of Deaf people who are ready to join the workforce. As more Deaf people join the workforce, new Deaf CoPs could emerge, and students could learn from these communities.

\section{Conclusion}

During the course of the past three years, I had the opportunity to reacquaint myself with the history and evolution of my (Deaf) community. At times while writing of this dissertation, I felt the negative influence of history on my community and language. When I read about the oppression of people in the Deaf community in the 1800s, and I realized the same oppression still exists today in many cases, it was difficult to continue 
moving forward with my studies. On those difficult days, I would take my place in front of a classroom of hearing students who enrolled in my class because they wanted to learn more about my language, my culture, and my community; they gave me the motivation and inspiration to continue to do my research.

I have worked at WOU in the ASL Studies department for 10 years, and I remember when I first started, there were no other full-time Deaf instructors. The only time I saw other Deaf people on campus was when part-time adjunct instructors came to teach class. Though I am Deaf and grew up in the Deaf community, I had a difficult time finding a local Deaf community at or near WOU. It was difficult for me, a native member of the Deaf community, to find connections and CoPs, which means it is that much more difficult for students to find connections, language acquisition, and apprenticeship opportunities.

I realized immediately, that for students to improve their language skills, they needed to have exposure to native language models more often. Fortunately, the division chair and the dean (at that time) were supportive of my request to hire more full-time Deaf instructors. Now, 10 years later, students report that the full-time Deaf instructors at WOU seem to be their own Deaf CoP, and in so many ways, that portrays an accurate frame. My colleagues have become my community, and my connection with them has made WOU a safe place for me to try new ideas, support students in their LPP and apprenticeship opportunities, and to continue to challenge old frames in the interest of finding new ones that work better. 
I am excited to build on my dissertation research, and I am anxious to see if there are parallels in other locations that have small Deaf populations/communities. I truly believe that with concentrated effort, we can find a way for both communities to benefit one another. I cannot stress enough the importance of exposure to native language models for language acquisition. While it is too soon to know whether the new flipped frame model of ASL/Deaf community events will be effective at WOU, my hope is that these events will help address some of the underlying issues that have plagued our program and its students for so many years.

As a generationally Deaf woman, whose first language is ASL, I am beyond proud to complete this dissertation and my doctoral degree. For many years, I believed I would be unable to complete a doctoral program because English was my second language. That fear led me to believe that hearing people were better able to capture research (e.g., articles, book chapters, dissertations) about ASL and the Deaf community than I was. It was not until I faced the reality that a doctorate was essential if I wanted to invest in the ASL Studies program at WOU (or any university) and help make decisions about the program's growth and trajectory.

The number of Deaf researchers, scholars, and doctoral students and candidates continues to grow, but this number is still much lower than our hearing counterparts. I am proud to stand with fellow Deaf colleagues, family members, and friends, who have completed their degrees and who continue to engage in teaching, research, and scholarship about our language, community, and culture. It is my hope that Deaf people 
will read this research and will realize that they too have a unique lens and story to tell, and that this realization will be enough to push them forward. 


\section{References}

Anderson, J., Reader, L., \& Simon, H. (1996). Situated learning and education. Educational Researcher, 25(4), 5-11. doi:10.3102/0013189X025004005

Armstrong, D. (2000). William C. Stokoe, Jr. founder of sign language linguistics 19192000. Retrieved from http://gupress.gallaudet.edu/stokoe.html

Brown, J. S., Collins, A., \& Duguid, S. (1989). Situated cognition and the culture of learning. Educational Researcher, 18(1), 32-42.

Cawthon, S., Garberoglio, C., \& Bond, M. (2016). Deaf people and employment in the United States: 2016. Retrieved from https://www.nationaldeafcenter.org/sites/ default/files/Deaf\%20Employment\%20Report_final.pdf

Cokley, D., \& Baker-Shenk, C. L. (1991). American Sign Language: A student text, units 1-9. Washington, DC: Clerc Books.

Cornell-Swanson, L.V. (2001). The transmission of culture in an American Sign Language (ASL) immersion classroom (Doctoral dissertation). Retrieved from ProQuest Digital Dissertations. (3008802)

Creswell, J. W. (2014). Research design: Qualitative, quantitative, and mixed methods approaches ( $4^{\text {th }}$ ed.). Los Angeles, CA: Sage.

Devlin, K. (2015). Learning a foreign language a "must" in Europe, not so in America. Pew Research Center, 13. Retrieved from https://www.pewresearch.org/ fact-tank/ 2015/07/13/learning-a-foreign-language-a-must-in-europe-not-so-inamerica/

Edwards, R. (2007). Chasing Aleck: The story of a dorm. The Public Historian, 29(3), 87-104. doi:10.1525/tph.2007.29.3.87

Emmorey, K. (1993). Processing a dynamic visual—Spatial language: Psycholinguistic Studies of American Sign Language. Journal of Psycholinguistic Research, 22(2), $153-187$.

Gannon, J. R. (1981). Deaf heritage. Silver Spring, MD: National Association of the Deaf.

Gilardi, S., \& Guglielmetti, C. (2011). University life of non-traditional students: Engagement styles and impact on attrition. Journal of Higher Education, 82, $33-53$. 
Girolamo Cardano. (2018). Encylopedia Britannica. Retrieved from https://www.britannica.com/biography/Girolamo-Cardano

Goldberg, D., Looney, D., \& Lusin, N. (2015). Enrollments in languages other than English in United States institutions of higher education, Fall 2013. Retrieved from https://www.mla.org/content/download/31180/1452509/ EMB_enrllmnts_nonEngl_2013.pdf

Gregg, K. R. (1984). Krashen's monitor and Occam's razor, Applied Linguistics, 5, 79-100.

Hodkinson, P., \& Hodkinson, H. (2004). The significance of individuals' dispositions in workplace learning: A case study of two teachers. Journal of Education and Work, 17(2), 167-182.

Holcomb, T. (2017, June). Required Deaf event attendance: A burden on the Deaf community? Paper presented at the annual conference of the American Sign Language Teachers Association, Salt City, UT.

Holcomb, T. (2018). Endnote address. Presentation at the Conference of Interpreter Trainers (CIT). [Video stream]. Retrieved from https://streetleverage.com/tomholcomb-endnote-address-cit-2018/

Houston, T. J. (2018). Defining academia influences on mobility, identity, and culture of Deaf scholars in higher education (Doctoral dissertation). Retrieved from ProQuest Digital Dissertations. (10830314)

Jacobowitz, E. L. (2005). American Sign Language teacher preparation programs in the United States. Sign Language Studies, 8(1), 76-110.

Jankowski, K. A. (1997). Deaf empowerment. Washington, DC: Gallaudet University Press.

Kemp, M. (1998). Why is learning American sign language a challenge? American Annuals of the Deaf, 143(3), 255-259. Retrieved from https://muse.jhu.edu/ login?auth=0\&type=summary\&url=/journals/american_annals_of_the_deaf/v143/ 143.3kemp.html

Knapik, M. (2006). The qualitative research interview: Participants' responsive participation in knowledge making. International Journal of Qualitative Methods, 5(3), 77-93.

Krashen, S. D. (1982). Principles and practice in second language acquisition. New York, NY: Pergamon. 
Krashen, S. D. (1988). Second language acquisition and second language learning. Upper Saddle River, NJ: Prentice-Hall International.

Lane, H., Hoffmeister, R., \& Bahan, B. (1996). A journey into the Deaf-world. San Diego, CA: Dawn Sign Press.

Lave, J., \& Wenger, E. (1991). Situated learning: Legitimate peripheral participation. Cambridge, UK: Cambridge University Press.

Liu, D. (2015). A critical review of Krashen's Input Hypothesis: Three major arguments. Journal of Education and Human Development, 4(4), 139-146.

Mathers, C. M., \& Witter-Merithew, A. (2014). The contribution of Deaf interpreters to GATEKEEPING within the interpreting profession: Reconnecting with our roots. Proceedings from the Biennial Conference-Our Roots: The Essence of Our Future Conference (pp. 158-173), Portland, OR.

Maxwell, J. A. (2013). Qualitative research design: An interactive approach ( $\left.3^{\text {rd }} \mathrm{ed}.\right)$. Thousand Oaks, CA: Sage.

McKee, D., \& McKee, R. L. (1992). What's so hard about learning ASL?: Students' \& teachers' perceptions. Sign Language Studies, 75(1), 129-157.

McLaughlin, B. (1987). Theories of second-language learning. London, England: Edward Arnold.

Merriam, S. B., \& Tisdell, E. J. (2016). Qualitative research: A guide to design and implementation ( $4^{\text {th }}$ ed.). San Francisco, CA: Jossey-Bass.

Morgan, S. (2014, December 8). National Center on Deafness celebrates 50 years of exemplary service. CSUN Today. Retrieved from https://csunshinetoday.csun.edu/ university-news/national-center-on-deafness-celebrates-50-years-of-exemplaryservice/

National Collaborative on Workforce and Disability. (2016). Attitudinal barriers for people with disabilities. Retrieved from http://www.ncwd-youth.info/ publications/attitudinal-barriers-for-people-with-disabilities/

Quinto-Pozos, D., \& Mehta, S. (2010). Register variation in mimetic gestural complements to signed language. Journal of Pragmatics, 42(3), 557-584.

Padden, C., \& Humphries, T. (2005). Inside deaf culture. Cambridge, MA: Harvard University Press.

Schunk, D. H. (2008). Learning theories: An educational perspective. Upper Saddle River, NJ: Pearson Prentice Hall. 
Seidman, I. (2013). Interviewing as qualitative research: A guide for researchers in the social sciences $\left(4^{\text {th }}\right.$ ed.). New York, NY: Teachers College Press.

Smith, C., Lentz, E. M., \& Mikos, K. (2008). Signing naturally. San Diego, CA: Dawn Sign Press.

Stokoe, W. C., Jr. (2005). Sign language structure: An outline of the visual communication systems of the American deaf. Journal of Deaf Studies and Deaf Education, 10(1), 3-37.

Vygotsky, L. (1978). Interaction between learning and development. In M. Gauvin \& M. Cole (Eds.), Readings on the development of children (pp. 34-41). New York, NY: Scientific American Books.

Which colleges grant the most bachelor's degrees in foreign languages? (2019).

Chronicle of Higher Education, 65(21). Retrieved from https://www.chronicle. com/article/Which-Colleges-Grant-the-Most/245567

White, L. (1987). Against comprehensible input: The input hypothesis and the development of second language competence, Applied Linguistics, 8, 95-110.

Wilcox, S., \& Wilcox, P. P. (1997). Learning to see: Teaching American Sign Language as a second language. Washington DC: Gallaudet University Press.

Yang, S. K., \& Kim, T. Y. (2011). Sociocultural analysis of second language learner. A qualitative case study of two study-abroad ESL learners. System: An International Journal of Educational Technology and Applied Linguistics, 39(3), 325-334.

Yin, R, K. (2018). Case study research and applications: Design and methods (6 $^{\text {th }}$ ed.). Thousand Oaks, CA: Sage. 
Appendix A

Recruitment Materials 


\section{Engaging In a Rural Deaf Community of Practice}

\section{Hello Students,}

My name is Kara Gournaris and I am an ASL instructor in the ASL Studies program at Western Oregon University (WOU). I am currently a doctoral student at Portland State University (PSU), and my area of research is focused on how hearing students interact with local Deaf communities of practice. If you are receiving this email, it means you are enrolled in ASL 8 during the winter 2019 term, and you are eligible to apply for participation in my dissertation research study. Participation consists of a 1:1 interview session with me (approximately 60minutes) that explores your experience learning ASL and engaging with Deaf community members. If you prefer to participate in this interview using spoken English, an ASL interpreter will be provided.

Please let me know if you are interested in learning more or participating in my dissertation research study. You can return this paper to me or contact me at gournark@wou.edu. Thank you for considering participation in my study. Your input would be so valuable.

Thank you!

Kara Gournaris

I am interested in learning more or participating in a study about engaging in a rural Deaf community of practice. 


\section{Appendix B}

Consent to Participate in Research 


\section{The Portland State University Consent to Participate in Research \\ Engaging In a Rural Deaf Community of Practice}

January 21, 2019

\section{Introduction}

You are being asked to participate in a research study that is being done by Micki Caskey who is the Principal Investigator and Kara Gournaris, from the Department of Curriculum and Instruction in the College of Education (COE) at Portland State University in Portland, Oregon. This research is studying students' beliefs and attitudes about participation in Deaf Communities of Practice (CoP).

You are being asked to participate in this study because you are enrolled in third year ASL courses at Western Oregon University, and have expressed interest in working in a Deaf-related field in the future. This form will explain the research study, and will also explain the possible risks as well as the possible benefits to you. We encourage you to talk with your family and friends before you decide to take part in this research study. If you have any questions, please ask one of the study investigators.

\section{What will happen if I decide to participate?}

If you agree to participate, the following things will happen:

Your participation in this study will be in the form of an interview. This interview is expected to last 60 minutes and will be conducted in American Sign Language. If you prefer to participate in this interview using spoken English, an ASL interpreter will be provided. This interview will consist of 16 questions, and you may refuse to answer any question without penalty. Your answers will be recorded for data analysis purposes, but your name and identity will not be disclosed in the final research/dissertation report.

How long will I be in this study?

Participation in this study will take a total of 1 hour over a period of interview session.

What are the risks or side effects of being in this study?

There are risks of stress, emotional distress, inconvenience and possible loss of privacy and confidentiality associated with participating in a research study.

This study does not pose any unusual risks for the participants. Participants may be at minimal risk of emotional and psychological stress associated with participating in an interview. If they experience any stress during the interview, they can stop the interview at any time. Participants will also be assured that their responses will be used exclusively for study purpose and will not be shared with their instructors or peers. 
For more information about risks and discomforts, ask the investigator.

What are the benefits to being in this study?

Participants may benefit from having the opportunity to share their voices about their experiences with learning ASL. Higher education instructional and research faculty as well as other instructors of ASL may benefit from an exploration of university students' experiences with second language acquisition — in this case ASL_-including the supports and pedagogical practices that improve their learning.

How will my information be kept confidential?

We will take measures to protect the security of all your personal information, but we cannot guarantee confidentiality of all study data. Data will not linked to the participants' identity. The participants' identity will not be disclosed at any time or in any part of the dissertation research. The participants' responses will be used only for the dissertation research. The data will be de-identified prior to any publication including the dissertation. The participants' responses will not be shared with their instructors or other students. They will be used exclusively by the researcher for the purpose of the dissertation research. All data files will be saved and stored in an electronic program called Smartsheets. This program is a password protected site that is designed similarly to Excel, and all content will be uploaded to this site during the project. Students' real names will not be used, and no other identifying information will be saved that might compromise participants' identities. This account and all data stored in it will be deleted upon the completion of this dissertation research.

Information contained in your study records is used by study staff and, in some cases it will be shared with the sponsor of the study. The Portland State University Institutional Review Board (IRB) that oversees human subject research and/or other entities may be permitted to access your records, and there may be times when we are required by law to share your information. It is the investigator's legal obligation to report child abuse, child neglect, elder abuse, harm to self or others or any life-threatening situation to the appropriate authorities, and; therefore, your confidentiality will not be maintained.

Your name will not be used in any published reports about this study.

Will I be paid for taking part in this study?

Participants will receive no compensation.

Can I stop being in the study once I begin?

Your participation in this study is completely voluntary. You have the right to choose not to participate or to withdraw your participation at any point in this study without penalty or loss of benefits to which you are otherwise entitled.

Whom can I call with questions or complaints about this study? 
If you have any questions, concerns or complaints at any time about the research study, Micki Caskey will be glad to answer. You can reach Micki at 503-725-4749 or caskeym@pdx.edu.

If you need to contact someone after business hours or on weekends, please call 503807-7814 and ask for Micki Caskey.

Whom can I call with questions about my rights as a research participant?

If you have questions regarding your rights as a research participant, you may call the PSU Office for Research Integrity at (503) 725-2227 or 1(877) 480-4400. The ORI is the office that supports the PSU Institutional Review Board (IRB). The IRB is a group of people from PSU and the community who provide independent oversight of safety and ethical issues related to research involving human participants. For more information, you may also access the IRB website at https://sites.google.com/a/pdx.edu/research/integrity.

\section{CONSENT}

You are making a decision whether to participate in this study. Your signature below indicates that you have read the information provided (or the information was read to you). By signing this consent form, you are not waiving any of your legal rights as a research participant.

You have had an opportunity to ask questions and all questions have been answered to your satisfaction. By signing this consent form, you agree to participate in this study. A copy of this consent form will be provided to you.

Name of Adult Subject (print)

Signature of

\section{INVESTIGATOR SIGNATURE}

This research study has been explained to the participant and all of his/her questions have been answered. The participant understands the information described in this consent form and freely consents to participate.

Name of Investigator/ Research Team Member (type or print)

(Signature of Investigator/ Research Team Member) Date 
Appendix C

Individual Interview 
Interview Introduction Script

Thank you for agreeing to participate in this research study. I designed the following interview questions to gather information about your experiences and interactions in the Deaf community. I also want to learn more about your experience during this study. There are no right or wrong answers to any of the questions we discuss. You can choose not to answer any question you don't feel comfortable answering. You may also choose to withdraw from this interview or research study at any time with no penalty or consequence.

I will be using a semi-structured interview protocol. After answering questions, I may decide to ask you a follow-up question to collect more information. At the conclusion of this research study, I will use this data as part of my dissertation research. Your name and all personal identifying information will be removed and will not be shared in any of my research findings.

I will conduct the interview in American Sign Language (ASL). You will respond to my questions in spoken English. Your answers will be interpreted to me in ASL. The interview will be recorded, and the audio file will be transcribed. You will be given a written transcription of the interview to review. During this review, you may clarify or change your responses. I will analyze the information you share and develop a case report. Your name and other personal identifying information will never be shared with anyone during this study. Let's begin with the following questions. 


\section{Demographic information:}

1. How long have you been using American Sign Language (ASL)?

2. What is your year in the ASL program?

3. Tell me about your prior experience with ASL prior to arriving to WOU.

\section{Community of practice definition:}

4. I am interested in learning about communities of practice for ALS students. What is your familiarity with the terminology "community of practice" $(\mathrm{CoP}) ?$

\section{Interview:}

[Research Question \#1. How do students in an American Sign Language (ASL) program at a rural west coast university describe opportunities to engage with native ASL communities of practice?]

5. Please describe your engagement with native ASL communities of practice.

a. What opportunities have made it is easy for you to engage with the native ASL community?

b. Have any past experiences made you confident to participate in the Deaf community? Please explain.

c. What barriers have made difficult for you to engage with the native ASL community?

a. Have any past experiences made you apprehensive to participate in the Deaf community? Please explain 
6. Why would you want to be part of the Deaf community/ASL community of practice Deaf?

7. Have you had many interactions with native ASL users (i.e., Deaf community members)? If yes, in what capacity? (e.g., school, on your own, family, work, other)

8. Do you have close family or friends who are an active part of the Deaf community? If yes, please elaborate.

[Research Question \#2. In what ways do students who are learning ASL at a rural west coast university feel part of the Deaf/native ASL community of practice?]

For the next questions, please look at this model of the Deaf community of practice

9. Describe where you are on this model of the Deaf community of practice.

a. How long do you think it will take you to move up to the next level? [Ask students to indicate which ring on my model and state how long it will take to make that transition.

10. Did you feel welcome at the events you attended or in your interactions with the Deaf community? Please explain your experiences.

11. In a community, there are different levels of engagement, involvement, and acceptance. If you had to identify your current place within the Deaf community, where would that be?

[Research Question \#3. What experiences helped students apprentice into the Deaf community?] 
12. Have you been to any Deaf events? If yes, how many events have you been to? Which events did you attend?

13. Did you feel welcome at the events you attended or in your interactions with the Deaf community? Please explain your experiences.

14. Are you interested in becoming a more active participant/community member in the Deaf community? If yes, how would you do that?

15. What does involvement in a Deaf community of practice mean to you? What does this look like?

16. Thinking about your personal and professional goals as well as the completion of your ASL coursework at WOU, where do you hope your place will be in the Deaf community of practice? Please elaborate. [Ask students to indicate which ring on my model.] 